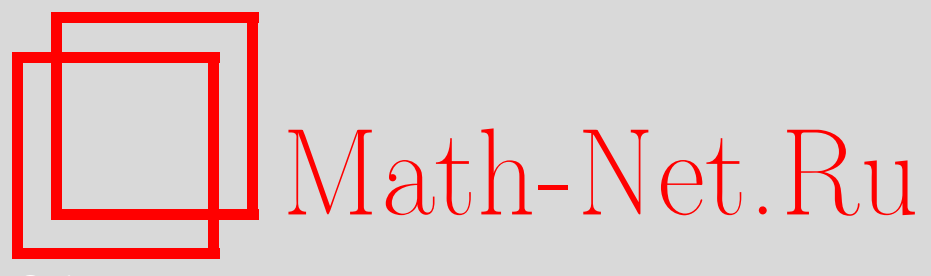

М. В. Карасев, Е. М. Новикова, Алгебра и квантовая геометрия многочастотного резонанса, Изв. РАН. Сер. матем., 2010, том 74, выпуск 6, 55-106

DOI: https://doi.org/10.4213/im4107

Использование Общероссийского математического портала Math-Net.Ru подразумевает, что вы прочитали и согласны с пользовательским соглашением http://www . mathnet.ru/rus/agreement

Параметры загрузки:

IP : 54.209 .52 .79

26 апреля 2023 г., 18:20:58

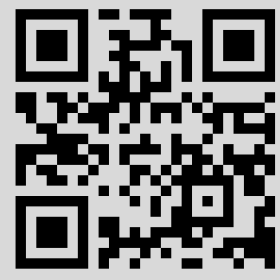


УДК $517.986+517.958$

М. В. Карасев, Е. М. Новикова

\title{
Алгебра и квантовая геометрия многочастотного резонанса
}

\begin{abstract}
Алгебра симметрий квантового резонансного осциллятора в случае трех и более частот описана с помощью конечного (минимального) числа образующих и полиномиальных соотношений. Для этой алгебры указаны конструкция квантовых листов с комплексной структурой (аналог классических симплектических листов), а также конструкции квантовой кэлеровой 2-формы, воспроизводящей меры, соответствующих неприводимых представлений и когерентных состояний.
\end{abstract}

Библиография: 40 наименований.

Ключевые слова: частотный резонанс, алгебра симметрий, нелинейные коммутационные соотношения, квантовые кэлеровы формы, когерентные состояния.

\section{§1. Введение}

В волновой и квантовой механике многомерных систем фундаментальную роль играют состояния, локализованные вблизи устойчивого положения равновесия [1]-[3]. Гармоническая часть таких систем - осциллятор - задает главную составляющую движения, в то время как ангармоническая часть представляет возмущение. После процедуры квантового усреднения это возмущение начинает коммутировать с гармонической частью, т.е. задает элемент из ее коммутанта (алгебры симметрий). Если частоты гармонической части находятся в резонансе, то алгебра симметрий некоммутативна. Факт некоммутативности приводит к нетривиальной динамике усредненной системы и порождает ряд интересных эффектов.

В классической механике симметрии резонансного осциллятора изучались давно (см., например, [4], [5] и ссылки в фундаментальном обзоре [6]). Пуассонова структура на пространстве симметрий была исследована для некоторых частных случаев резонанса, например, в [7]-[9].

В работе [10] было показано, что алгебра симметрий общего резонансного осциллятора является алгеброй с конечным числом образующих и полиномиальными соотношениями. Она была названа резонансной алгеброй.

Гармоническая часть исходной системы представляет элемент Казимира (центр) резонансной алгебры. Усредненная ангармоническая часть задает еще один гамильтониан на резонансной алгебре. В устойчивом случае, когда все резонансные частоты положительны, этот гамильтониан соответствует некоторой возникшей за счет резонанса квазичастице, которую мы называем гироном. Оказалось, что описание самой резонансной алгебры и анализ состояний

Работа выполнена при финансовой поддержке РФФИ (грант № 09-01-00606-а).

(C) M. B. Kapaceb, Е. М. Новикова, 2010 
(прецессии) гирона сопровождаются появлением необычной квантовой геометрии [11]-[14]. Обнаруженные здесь новые алгебраические и геометрические объекты позволили решить давно стоявшую проблему об асимптотике спектра и волновых состояний в нано- и микрозонах вблизи резонансов.

Рассматриваемая задача о резонансах имеет два важных аспекта. Во-первых, она охватывает широкий круг базовых моделей волновой оптики и квантовой нанофизики. Во-вторых, как оказалось, она доставляет интересный класс алгебр, заданных конечным числом образующих с полиномиальными, в общем случае нелинейными соотношениями, для которых возможно построение полной теории неприводимых представлений, включая обобщение на базе квантовой геометрии известного метода орбит (разработанного для случая алгебр Ли [15]).

Систематическое рассмотрение квантовых алгебр с нелинейными соотношениями было начато школами В.П. Маслова и Л.Д. Фаддеева [16]-[25] в 1970-1980-х годах, хотя первые попытки использования таких алгебр делались физиками намного раньше.

Долгое время список физических систем, в которых алгебры с нелинейными соотношениями играют существенную роль для описания спектра и динамики, сводился к бесконечномерным полевым системам и спиновым цепочкам (см. список литературы в [22]). Примеры нелиевских алгебр с конечным числом образующих, свойства которых проявляются в фундаментальных эффектах квантовой механики (эффекты Зеемана и Зеемана-Штарка), были обнаружены и подробно изучены в работах [26]-[28]. По своему типу эти семейства алгебр подобны тем алгебрам, которые рассматривались в работе [16], т. е. число образующих в них совпадает с размерностью спектра.

Обнаруженные в [26]-[28] алгебры являются квантовыми, т. е. деформациями некоторых классических пуассоновых алгебр (с полиномиальным пуассоновым тензором). Резонансные алгебры, отвечающие многочастотному квантовому осциллятору, которые были найдены в [11]-[13], также относятся к классу квантовых алгебр, но число их образующих, вообще говоря, уже больше размерности спектра.

Квантовый осциллятор с частотами $f_{1}, \ldots, f_{n}$ задается гамильтонианом

$$
\widehat{H}=-\frac{\hbar^{2}}{2} \Delta+\frac{1}{2}\left(f_{1}^{2} q_{1}^{2}+\cdots+f_{n}^{2} q_{n}^{2}\right)-\frac{\hbar}{2}\left(f_{1}+\cdots+f_{n}\right) .
$$

Этот оператор действует по переменным $q_{j}$ в пространстве $L^{2}\left(\mathbb{R}^{n}\right)$. Предполагаем, что параметр $\hbar$ больше нуля, и рассматриваем для простоты лишь устойчивый случай $f_{j}>0, j=1, \ldots, n$.

Определим операторы уничтожения

$$
\hat{z}_{j}=\sqrt{\frac{f_{j}}{2}} q_{j}+\frac{\hbar}{\sqrt{2 f_{j}}} \frac{\partial}{\partial q_{j}},
$$

а также рассмотрим их сопряженные $\hat{z}_{j}^{*}$. Выполнены соотношения

$$
\left[\hat{z}_{j}, \hat{z}_{k}\right]=0, \quad\left[\hat{z}_{k}, \hat{z}_{j}^{*}\right]=\hbar \delta_{j, k}
$$

Вводя операторы действия $\widehat{S}_{j}=\hat{z}_{j}^{*} \hat{z}_{j}, j=1, \ldots, n$, можно записать гамильтониан осциллятора в виде скалярного произведения вектора частот и 
вектор-оператора действия:

$$
\widehat{H}=\langle f, \widehat{S}\rangle .
$$

Операторы действия взаимно коммутируют, их совместный спектр образует решетку с шагом $\hbar$ в $n$-мерном пространстве: $\operatorname{Spectr}(\widehat{S})=\left\{\hbar k \mid k \in \mathbb{Z}_{+}^{n}\right\}$.

Исследование алгебры симметрий оператора $\langle f, \widehat{S}\rangle$ можно свести к исследованию простых резонансных случаев, когда набор частот целочисленный, $f \in \mathbb{N}^{n}$, и простой, т. е. любая пара частот не имеет нетривиального общего делителя (см., например, [10]). Мы рассматриваем именно такие резонансные случаи.

Из соотношений (1.2) следует, что алгебра симметрий резонансного осциллятора, т. е. алгебра операторов в $L^{2}\left(\mathbb{R}^{n}\right)$, коммутирующих с $\langle f, \widehat{S}\rangle$, порождается генераторами

$$
\left(\hat{z}^{*}\right)^{r} \hat{z}^{l}, \quad \text { где } \quad\langle f, l-r\rangle=0, \quad l, r \in \mathbb{Z}_{+}^{n} .
$$

Обратим внимание на то, что генераторы $\left(\hat{z}^{*}\right)^{r} \hat{z}^{l}$ не являются независимыми и подчинены ряду тождеств.

В классической механике функции $\bar{z}^{r} z^{l}$ с условием $\langle f, l-r\rangle=0$ называются резонансными нормальными формами (см. [5], [29]); они задают алгебру функций, находящихся в инволюции с классическим резонансным осциллятором. В [10] было показано, как из этого бесконечного семейства генераторов можно выделить конечный базис “минимальных" генераторов. В квантовом случае минимальный базис также существует.

После установления этого первого нетривиального факта возникает следующая задача: описать соотношения в ассоциативной алгебре, порожденной этим базисом. Иными словами, требуется задать алгебру симметрий резонансного осциллятора не через ее конкретное представление (минимальными) нормальными формами (1.4), а как абстрактную алгебру с конечным числом образующих и алгебраическими соотношениями. Мы называем ее резонансной алгеброй.

В рамках классической механики эту задачу можно переформулировать следующим образом: найти минимальное алгебраическое многообразие с алгебраической пуассоновой структурой и каноническое отображение на него стандартного фазового пространства $\mathbb{R}^{2 n}$, находящееся в инволюции с резонансным осциллятором $\langle f, S\rangle$. Поскольку гамильтоновы траектории - это окружности, мы приходим к задаче об алгебраическом описании пуассоновой редукции пространства $\mathbb{R}^{2 n}$ по действию окружности (см. детали об общей редукции по действию окружности, например, в [30]). В работе [10] было найдено решение этой задачи для случая общего резонанса. Другие подходы для некоторых вырожденных случаев резонанса развивались в [7], [9].

Отметим, что в пространстве частот можно задать естественное понятие арифметической эквивалентности по действию группы $\mathrm{SL}(n, \mathbb{Z})$ целочисленных матриц с единичным определителем. Однако для арифметически эквивалентных наборов частот соответствующие резонансные алгебры и их пуассоновы многообразия могут оказаться принципиально различными. Например, в двумерном случае любой набор взаимно простых частот арифметически эквивалентен набору единичных частот 1:1; при этом резонансная алгебра для первого набора задается, вообще говоря, нелинейными коммутативными соотношениями и не сводится ни к какой конечномерной алгебре Ли, а для второго 
набора резонансная алгебра - это простая трехмерная алгебра Ли su(2). Таким образом, из арифметической эквивалентности не вытекает алгебраическая эквивалентность. С другой стороны, наборы частот, отличающиеся только перестановкой, конечно, эквивалентны и арифметически, и алгебраически. Далее, набор частот с общим целочисленным множителем алгебраически эквивалентен набору, в котором этот множитель сокращен. Более того, любой набор частот в алгебраическом смысле всегда можно редуцировать к элементарным наборам, в которых частоты попарно взаимно просты (см. [10]). Именно такие элементарные наборы частот мы изучаем в настоящей работе.

Нельзя сказать, что квантовая версия задачи о резонансной алгебре просто дублирует классическую. Конечно, в двухчастотном случае описание квантовой резонансной алгебры достаточно простое, оно было получено в [12], [13] параллельно с описанием классической алгебры. Однако случай квантового многочастотного резонанса при $n \geqslant 3$ оказался намного сложнее своей классической версии.

Случаи трех и более частот принципиально отличаются от двухчастотного тем, что резонансная решетка векторов "рождения" (переходов между точками спектра операторов действия) имеет аномалию [10]. В настоящей работе мы показываем, что в этой аномальной решетке можно выделить максимальные нормальные подрешетки. Они играют существенную роль в описании как пуассонова тензора, так и квантовых соотношений резонансной алгебры. Эти же подрешетки участвуют в конструкции когерентных преобразований; здесь нормальность подрешеток снимает проблему упорядочения операторов "рождения".

Существование в многочастотном случае некоммутирующих "рождений” заставляет ввести [10] понятие коммутатора между векторами целочисленной решетки. Нормальные резонансные подрешетки можно иначе определить как максимальные коммутативные подрешетки. Коммутатор между векторами решетки задает скобки между координатами "рождение"-"рождение" и "рождение"-“уничтожение" в резонансной пуассоновой алгебре.

В квантовом многочастотном случае приходится вводить еще целый ряд новых операций на целочисленной резонансной решетке. В этом случае для описания резонансной алгебры в большей степени привлекается арифметика. В частности, здесь возникают интересные "структурные полиномы" на целочисленной решетке, которые играют роль структурных функций в квантовых перестановочных соотношениях.

Рассматриваемая задача, помимо описания резонансной алгебры, содержит в себе еще и подзадачу: построение неприводимых представлений этой алгебры. Здесь неожиданно оказывается, что использовать классические геометрические объекты (пуассонов тензор, симплектические листы) для построения квантовых неприводимых представлений в прямой аналогии с методом орбит не удается. Например, размерность квантового неприводимого представления нельзя выразить через классический объем Лиувилля симплектических листов.

В общей схеме квантования известно, что размерность определяется воспроизводящей мерой на квантовом листе, и над ним же строятся неприводимые представления алгебры [31], [32]. В настоящей работе мы даем явное описание квантовых листов для многочастотного осциллятора. Конструкция основана на групповой структуре резонансной решетки, позволяющей ввести универ- 
сальное семейство многообразий с комплексной структурой, которое задается лишь резонансными частотами и собственными числами осциллятора. С одной стороны, эти многообразия могут быть вложены в резонансное пуассоново многообразие в качестве замыкания симплектических листов. Тем самым, на них определена классическая замкнутая 2-форма Кириллова (с сингулярностями). Она является кэлеровой по отношению к комплексной структуре, но для нее нет воспроизводящей меры. С другой стороны, на этих же многообразиях можно ввести квантовую кэлерову 2 -форму без сингулярностей ${ }^{1}$, для которой имеется воспроизводящая мера. Данная мера генерирует формулу для размерности неприводимого представления резонансной алгебры, а также задает гильбертову структуру в пространстве, где действует представление. При этом операторы неприводимого представления конструируются явно в виде дифференциальных операторов с полиномиальными коэффициентами. Порядок этих операторов, вообще говоря, больше единицы. Это соответствует тому факту, что соотношения в алгебре нелинейные, и при этом комплексная поляризация не инвариантна. Только в случае изотропного резонанса, когда все частоты равны единице, резонансная алгебра оказывается алгеброй Ли, поляризация инвариантна и операторы неприводимого представления имеют порядок 1.

Введенные квантовую 2-форму и соответствующую воспроизводящую меру мы называем объектами квантовой геометрии. В классическом пределе, вне точек сингулярности, эти объекты переходят соответственно в классическую форму Кириллова и меру Лиувилля на симплектических листах, но в окрестности сингулярностей (на замыкании листов) такого перехода нет. В принципе, можно было бы попытаться обойтись в этой задаче и без квантовой геометрии, используя лишь классические листы и общее геометрическое квантование для случая неинвариантной поляризации и следуя схеме из [34], но при этом вся конструкция была бы на порядок сложнее, операторы неприводимого представления содержали бы сингулярности и были бы псевдодифференциальными, а не дифференциальными, как в нашем варианте (см. обсуждение в [14], [31], [32]).

В настоящей работе мы предъявляем также простую формулу для когерентных преобразований, сплетающих исходное представление алгебры симметрий резонансного осциллятора с неприводимыми представлениями в пространстве антиголоморфных сечений эрмитова линейного расслоения над квантовыми листами. Отметим, что построенные когерентные состояния резонансного осциллятора позволяют также дать реализацию представлений резонансной алгебры в пространстве функций над лагранжевыми подмногообразиями в квантовом листе; она удобна для вычисления квазиклассических асимптотик (см. [13], [14]). В этой реализации, в частности, участвует фазовая функция (действие), порожденная квантовой 2-формой.

\section{§ 2. Минимальные резонансные векторы}

Решеткой мы будем называть подмножество векторов в $\mathbb{R}^{n}$ с целочисленными декартовыми координатами, которое является полугруппой относительно сложения.

1 Эта форма замкнута, но может вырождаться, т. е. она доставляет пример вихревой структуры [33]. 
Фиксируем вектор частот $f \in \mathbb{N}^{n}$. Резонансная решетка $\mathcal{R}=\mathcal{R}[f]$ определяется как множество целочисленных векторов из $\mathbb{R}^{n}$, ортогональных вектору частот. Таким образом, $\sigma \in \mathcal{R}$, если все координаты $\sigma_{j}$ целые и

$$
\langle f, \sigma\rangle=0 \text {. }
$$

Такие векторы $\sigma$ мы называем резонансными.

Пересечение резонансной решетки $\mathcal{R}$ с декартовыми квадрантами назовем нормальными подрешетками. На каждой такой подрешетке ни одна из декартовых координат не меняет знак. Эти подрешетки можно индексировать следующим образом:

$$
\mathcal{R}^{j}, \mathcal{R}^{j k}, \mathcal{R}^{j k l}, \ldots
$$

Здесь индексы отмечают номера тех декартовых координат, которые должны быть неотрицательными (а остальные должны быть неположительными). На пересечениях этих подрешеток соответствующие координаты тождественно равны нулю; скажем, на пересечении $\mathcal{R}^{j} \cap \mathcal{R}^{j k}$ координата с номером $k$ равна нулю, координата с номером $j$ неотрицательна, а остальные координаты неположительны.

Общее количество нормальных резонансных подрешеток (2.1) задается формулой

$$
C_{n}^{1}+C_{n}^{2}+C_{n}^{3}+\cdots+C_{n}^{n-1}=2^{n}-2 .
$$

Вместе они покрывают всю резонансную решетку $\mathcal{R}$.

При $n=2$ нормальных решеток всего две: $\mathcal{R}^{1}$ и $\mathcal{R}^{2}$. Они состоят из целочисленных двумерных векторов, лежащих на прямой, ортогональной вектору частот $f \in \mathbb{N}^{2}$, либо по одну, либо по другую сторону от начала координат 0.

При $n=3$ нормальных резонансных подрешеток всего шесть:

$$
\mathcal{R}^{1}, \mathcal{R}^{2}, \mathcal{R}^{3}, \mathcal{R}^{12}, \mathcal{R}^{23}, \mathcal{R}^{31} \subset \mathbb{Z}^{3} .
$$

Они соответствуют шести секторам, на которые декартовы плоскости делят резонансную плоскость, ортогональную вектору частот $f \in \mathbb{N}^{3}$.

ОПРЕДЕЛЕНИЕ 2.1. Ненулевой резонансный вектор назовем минималъным, если его нельзя получить сложением двух ненулевых векторов из нормальной подрешетки.

Множество $\mathcal{M}$ всех минимальных векторов естественно рассматривать как множество элементарных образующих резонансной решетки. В несколько иных терминах понятие минимального вектора было введено в [10]. Там же была доказана теорема о полноте: любой резонансный вектор представи́м в виде линейной комбинации минимальных векторов с натуральными коэффициентами. Этот результат можно уточнить.

Подмножество резонансной решетки назовем нормальным, если оно лежит в одной из нормальных резонансных подрешеток.

Резонансный вектор назовем внутренним, если он принадлежит только одной нормальной подрешетке. В противном случае этот вектор назовем граничHous.

Теорема 2.1. Любой резонансный вектор разлагается в сумму минимальных с неотрицательными иелыми коэффициентами:

$$
\sigma=\sum_{\varkappa \in \mathcal{M}_{\sigma}} n_{\varkappa}^{\sigma} \varkappa, \quad n_{\varkappa}^{\sigma} \in \mathbb{Z}_{+}, \quad \mathcal{M}_{\sigma} \subset \mathcal{M} .
$$


Здесъ $\mathcal{M}_{\sigma}$ - множество минимальных векторов в пересечении всех нормальных подрешеток, которым принадлежит вектор $\sigma$. В частности, $\mathcal{M}_{\sigma}-$ нормальное подмножество.

ДокАЗАТЕЛьство. Пусть вектор $\sigma$ не минимальный. Тогда он разлагается в сумму: $\sigma=\varkappa^{\prime}+\varkappa^{\prime \prime}$, где $\varkappa^{\prime}, \varkappa^{\prime \prime}$ лежат в той нормальной подрешетке, которой принадлежит $\sigma$. Если оба вектора $\varkappa^{\prime}$ и $\varkappa^{\prime \prime}$ минимальные, то доказательство закончено, если же хоть один из них не минимальный, то опять разлагаем его в сумму двух векторов (из той же нормальной решетки), и т. д. Процедура закончится за конечное число шагов, поскольку на каждом шаге целочисленные компоненты разлагаемого вектора представляются в виде суммы целых чисел того же знака и нулей (по определению нормальной подрешетки).

Отметим, что разложение (2.2), вообще говоря, не единственно.

Например, в случае трех частот $f_{1}=1, f_{2}=2, f_{3}=3$ резонансный вектор $\sigma=(2,-4,2)$ можно разложить в сумму минимальных векторов двумя способами:

$$
\sigma=2 \varkappa, \quad \sigma=\varkappa^{\prime}+\varkappa^{\prime \prime},
$$

где $\varkappa=(1,-2,1), \varkappa^{\prime}=(2,-1,0), \varkappa^{\prime \prime}=(0,-3,2)$.

Далее, количество слагаемых в разложении (2.2) не превосходит общего числа $M \stackrel{\text { def }}{=} \# \mathcal{M}$ минимальных резонансных векторов. В работе [10] было доказано, что $M$ конечно.

Для случая $n=3$ мы дадим явное описание множества минимальных резонансных векторов.

Прежде всего, заметим, что достаточно рассмотреть лишь случай взаимно простых частот. Действительно, если какие-то две частоты, скажем $f_{1}$ и $f_{2}$, имеют общий множитель:

$$
f_{1}=m f_{1}^{\prime}, \quad f_{2}=m f_{2}^{\prime},
$$

то ситуация легко редуцируется к новому набору частот $f_{1}^{\prime}, f_{2}^{\prime}, f_{3}$. Редукция происходит следующим образом. Сначала все сводится к случаю, когда частота $f_{3}$ взаимно проста с $m$. Затем для этого случая используем следующий факт: вектор $\left(\sigma_{1}, \sigma_{2}, \sigma_{3}\right)$ минимальный резонансный для набора частот $\left(f_{1}, f_{2}, f_{3}\right)$ тогда и только тогда, когда вектор $\left(\sigma_{1}, \sigma_{2}, \sigma_{3}\right)$ минимальный резонансный для набора частот $\left(f_{1}^{\prime}, f_{2}^{\prime}, f_{3}\right)$.

Таким образом, изучение, например, резонанса $3: 6: 2$ редуцируется к изучению резонанса ${ }^{2} 1: 1: 1$.

Для простоты в настоящей работе мы ограничиваемся рассмотрением случая положительных частот. Рассмотрение вариантов, в которых часть частот отрицательна, проводится аналогично. Итак, мы всюду ниже предполагаем, что выполнены следующие условия:

(А) частоты попарно взаимно простые;

(В) все частоты положительные.

Рассмотрим диофантово уравнение

$$
\mu f_{1}+\nu f_{2}+f_{3}=0
$$

\footnotetext{
${ }^{2}$ Резонансы именно такого вырожденного типа, редуцируемые к резонансу единичных частот, рассматривались в [9].
} 
относительно неизвестных целых чисел $\mu$ и $\nu$. Используем следующее простое утверждение.

Лемма 2.1. Существует единственное решение уравнения (2.3) с условием

$$
0 \leqslant \nu \leqslant f_{1}-1
$$

$B$ частности, если $f_{1}=1$, то это решение имеет вид $\mu=-f_{3}, \nu=0$. Если $f_{1} \geqslant 2$, mo $\nu \geqslant 1$.

Если $f_{1} \geqslant 2$, то для каждого $l=1,2, \ldots, f_{1}-1$ обозначим

$$
\nu^{(l)}=l \nu\left(\bmod f_{1}\right), \quad \mu^{(l)}=-\frac{l f_{3}+\nu^{(l)} f_{2}}{f_{1}},
$$

где $\nu$ - решение диофантова уравнения (2.3) с условием $(2.4)$. Числа $\mu^{(l)}, \nu^{(l)}$ целые, причем $0 \leqslant \nu^{(l)} \leqslant f_{1}-1$. Конечно, при $l=1$ имеем $\mu^{(1)}=\mu, \nu^{(1)}=\nu$.

Tеорема 2.2. В трехчастотном случае с условиями (A), (В) минимальные векторы в резонансной решетке $\mathcal{R}=\mathcal{R}^{23} \cup \mathcal{R}^{31} \cup \mathcal{R}^{12} \cup \mathcal{R}^{1} \cup \mathcal{R}^{2} \cup \mathcal{R}^{3}$ устроены следующим образом:

а) если $f_{1}=1$, то внутренних минимальных векторов в подрешетке $\mathcal{R}^{23}$ не существует;

b) если $f_{1} \geqslant 2$, то все внутренние минимальные векторы в подрешетке $\mathcal{R}^{23}$ задаются последовательностъю

$$
\left(\mu^{(l)}, \nu^{(l)}, l\right), \quad l=1, \ldots, f_{1}-1,
$$

причем вектор с номером l сохраняется в последовательности (2.5) только при условии, что

$$
\nu^{(l)}<\nu^{(j)}, \quad j=1, \ldots, l-1 .
$$

Граничные минимальные векторы в $\mathcal{R}^{23}$ имеют вид

$$
\left(-f_{3}, 0, f_{1}\right) \in \mathcal{R}^{23} \cap \mathcal{R}^{3}, \quad\left(-f_{2}, f_{1}, 0\right) \in \mathcal{R}^{23} \cap \mathcal{R}^{2} .
$$

Минимальные векторы нормальных подрешеток $\mathcal{R}^{31}$ и $\mathcal{R}^{12}$ получаются из предъдущего описания векторов в $\mathcal{R}^{23}$ ииклической перестановкой индексов $1,2,3$. Минимальные векторы в нормальной подрешетке $\mathcal{R}^{j}$ имеют вид $(-\sigma)$, где $\sigma$-минимальный вектор в подрешетке $\mathcal{R}^{k l}, k$ u $l$-номера, дополняющие номер ј до тройки индексов 1,2,3.

ДокАЗАТЕЛЬСтво приведено в работе [35].

В силу условия (2.6) все числа $\nu^{(l)}$ в последовательности (2.5) должны быть меньше $\nu$. В силу взаимной простоты $f_{1}$ и $f_{3}$ последовательность $(2.5)$ не может содержать два вектора с одной и той же второй координатой. Поэтому количество членов последовательности (2.5) не превосходит $\nu \leqslant f_{1}-1$. Это дает оценку сверху для числа внутренних минимальных векторов подрешетки $\mathcal{R}^{23}$. Аналогичные оценки получаются для подрешеток $\mathcal{R}^{31}$ и $\mathcal{R}^{12}$ (но частота $f_{1}$ заменяется на $f_{2}$ или $f_{3}$ ). Таким образом, общее число внутренних минимальных векторов не превосходит $2\left[\left(f_{1}-1\right)+\left(f_{2}-1\right)+\left(f_{3}-1\right)\right]=2\left(f_{1}+f_{2}+f_{3}\right)-6$, а граничных минимальных векторов (см. (2.7)) всего шесть.

СледСТВИЕ 2.1. В трехчастотном случае, $n=3$, число минимальных резонансных векторов имеет следуюшую верхнюю оценку:

$$
M \leqslant 2\left(f_{1}+f_{2}+f_{3}\right) .
$$




\section{§ 3. Пуассонова алгебра симметрий резонансного осциллятора}

Следуя работе [10], опишем резонансную алгебру классического осциллятора

$$
H=\frac{1}{2}\left(p_{1}^{2}+\cdots+p_{n}^{2}+f_{1}^{2} q_{1}^{2}+\cdots+f_{n}^{2} q_{n}^{2}\right)
$$

в эллиптическом случае (см. условие (В) в $§ 2)$.

На решетке $\mathbb{Z}^{n}$ введем следующие операции (индекс $j$ пробегает все значения $1, \ldots, n)$ :

$$
\begin{array}{ll}
\alpha \rightarrow|\alpha|, & |\alpha| \stackrel{\text { def }}{=}\left|\alpha_{1}\right|+\cdots+\left|\alpha_{n}\right|, \\
\alpha \rightarrow \theta(\alpha), & \theta(\alpha)_{j} \stackrel{\text { def }}{=} \begin{cases}1, & \text { если } \alpha_{j}>0, \\
0, \quad \text { если } \alpha_{j} \leqslant 0,\end{cases} \\
\alpha, \beta \rightarrow \alpha \cdot \beta, & (\alpha \cdot \beta)_{j} \stackrel{\text { def }}{=} \alpha_{j} \beta_{j}, \\
\alpha \rightarrow \alpha_{ \pm}, & \alpha_{ \pm} \stackrel{\text { def }}{=} \pm \alpha \cdot \theta( \pm \alpha), \\
\alpha, \beta \rightarrow \alpha \mid \beta, & (\alpha \mid \beta)_{j} \stackrel{\text { def }}{=} \min \left\{\left(\alpha_{-}\right)_{j},\left(\beta_{+}\right)_{j}\right\}, \\
\alpha, \beta \rightarrow[\alpha \mid \beta], & {[\alpha \mid \beta] \stackrel{\text { def }}{=} \alpha|\beta-\beta| \alpha,} \\
\alpha, \beta \rightarrow \alpha+\beta, & \alpha \stackrel{\circ}{+} \beta \stackrel{\text { def }}{=} \alpha|\beta+\beta| \alpha, \\
\alpha \rightarrow \stackrel{\circ}{\alpha}, & \stackrel{\circ}{\alpha} \stackrel{\text { def }}{=} \frac{1}{2}(\alpha \stackrel{\circ}{+}(-\alpha)) \equiv \frac{1}{2}\left(\alpha_{+}+\alpha_{-}\right), \\
\alpha, \beta \rightarrow \alpha \circ \beta, & \alpha \circ \beta=\circ \stackrel{\circ}{=} \alpha, \\
\alpha, \beta \rightarrow[\alpha, \beta], & {[\alpha, \beta] \stackrel{\text { def }}{=} \alpha \cdot \beta \beta_{-}-\alpha_{-} \cdot \beta_{+} \cdot}
\end{array}
$$

Еще одна полезная операция будет введена далее, в (3.18). Различные свойства операций (3.2) перечислены в [10]. Последняя из операций (3.2) была названа в [10] коммутатором. Операция $\stackrel{\circ}{+}$ была названа аномалией.

Отметим, что операция о, введенная в (3.2), ассоциативна, а коммутатор (3.2) представи́м в виде

$$
[\alpha, \beta]=\alpha \circ \beta-\beta \circ \alpha .
$$

Однако операция о не дистрибутивна по отношению к сложению по второму аргументу:

$$
\alpha \circ(\beta+\gamma)=\alpha \circ \beta+\alpha \circ \gamma-\alpha \cdot(\beta \stackrel{\circ}{+} \gamma)
$$

где $\stackrel{+}{+}$ обозначает аномалию. Поэтому "тождество Якоби" для коммутатора (3.3) имеет нестандартный вид (см. [10]).

Будем говорить, что векторы решетки коммутируют, если их коммутатор равен нулю. Простое вычисление показывает, что

$$
\alpha \stackrel{\circ}{+} \beta=0 \Longleftrightarrow[\alpha, \beta]=0 \Longleftrightarrow[\alpha \mid \beta]=0 .
$$

Лемма 3.1. Векторы из $\mathbb{Z}^{n}$ коммутируют тогда и только тогда, когда принадлежат одной нормальной подрешетке. Нормальная подрешетка может быть определена как максимальное коммутативное подмножество в данной решетке. 
ДоказАтельство. Сначала заметим, что

$$
[\alpha, \beta]=0 \quad \Longleftrightarrow \quad \alpha_{+} \cdot \beta_{-}=\alpha_{-} \cdot \beta_{+}=0 .
$$

Последнее (двойное) равенство означает, что соответствующие компоненты векторов $\alpha$ и $\beta$ не могут иметь разные знаки, т. е. эти векторы принадлежат одной и той же нормальной подрешетке.

Рассмотрим резонансную решетку $\mathcal{R}=\mathcal{R}[f]$ в $\mathbb{Z}^{n}$, порожденную набором частот $f \in \mathbb{N}^{n}$ осциллятора (3.1). Напомним, что $\mathcal{M}$ - подмножество в $\mathcal{R}$ минимальных резонансных векторов, а $\mathcal{M}_{\sigma}$ - нормальные подмножества в $\mathcal{M}$, по которым разлагаются резонансные векторы $\sigma$ согласно (2.2). В силу леммы 3.1 получаем

ПРЕДЛОЖЕНИЕ 3.1. Каждое подмножество $\mathcal{M}_{\sigma}$ коммутативно. Все элементы $\varkappa \in \mathcal{M}_{\sigma}$ в разложении (2.2) коммутируют друг с другом.

Рассмотрим пространство

$$
\mathcal{N}^{\#}=\mathbb{C}^{M} \times \mathbb{R}^{n}
$$

и определим на нем связи трех разных типов.

Напомним, что $M$ - число элементов в множестве $\mathcal{M}$. Комплексные координаты на первом сомножителе $\mathbb{C}^{M}$ в (3.5) будем нумеровать элементами $\sigma \in \mathcal{M}$; обозначим эти координаты через $A_{\sigma}$. Вещественные координаты во втором сомножителе $\mathbb{R}^{n}$ в $(3.5)$ обозначим через $S_{j}, j=1, \ldots, n$. Для любого $\alpha \in \mathbb{Z}_{+}^{n}$ обозначим $S^{\alpha}=S_{1}^{\alpha_{1}} \cdots S_{n}^{\alpha_{n}}$.

Связи эрмитова типа. Для любого минимального вектора $\sigma$ противоположный ему вектор $(-\sigma)$ соответствует сопряжению комплексной координаты:

$$
\bar{A}_{\sigma}=A_{-\sigma} .
$$

Связи коммутативного типа. Если два семейства $\{\rho\},\{\sigma\}$ коммутирующих минимальных векторов задают одинаковые линейные комбинации:

$$
\sum_{\rho} k_{\rho} \rho=\sum_{\sigma} m_{\sigma} \sigma, \quad k_{\rho}, m_{\sigma} \in \mathbb{N},
$$

то имеет место соотношение

$$
\prod_{\rho}\left(A_{\rho}\right)^{k_{\rho}}=\prod_{\sigma}\left(A_{\sigma}\right)^{m_{\sigma}} .
$$

ЗАмечание 3.1. Равенство (3.7) назовем приводимым, если коэффициенты $k_{\rho}, m_{\sigma}$ допускают представление в виде суммы: $k_{\rho}=k_{\rho}^{\prime}+k_{\rho}^{\prime \prime}, m_{\sigma}=m_{\sigma}^{\prime}+m_{\sigma}^{\prime \prime}$, такое, что $\sum_{\rho} k_{\rho}^{\prime} \rho=\sum_{\sigma} m_{\sigma}^{\prime} \sigma$, причем $k_{\rho}^{\prime}, k_{\rho}^{\prime \prime}, m_{\sigma}^{\prime}, m_{\sigma}^{\prime \prime} \in \mathbb{Z}_{+}$и $\left(\sum_{\rho} k_{\rho}^{\prime}\right)\left(\sum_{\rho} k_{\rho}^{\prime \prime}\right)$ не равно нулю. В остальных случаях будем говорить, что равенство (3.7) неприводимо. Нетрудно показать, что число неприводимых равенств (3.7), а значит, и число независимых связей коммутативного типа (3.8) конечны.

Связи некоммутативного типа. Если минимальные векторы $\rho$ и $\sigma$ не коммутируют и $\rho \neq-\sigma$, то выполнено соотношение

$$
A_{\rho} A_{\sigma}=S^{\rho+\sigma} \prod_{\varkappa \in \mathcal{M}_{\rho+\sigma}} A_{\varkappa}^{n_{\varkappa}^{\rho+\sigma}},
$$


где $\stackrel{\circ}{+}-$-аномалия, введенная в $(3.2), n_{\varkappa}^{\rho+\sigma}-$ коэффициенты разложения $(2.2)$ вектора $\rho+\sigma$ по минимальным векторам из $\mathcal{M}_{\rho+\sigma}$. Отметим, что если $\rho$ и $\sigma$ коммутируют, то соотношение (3.9) вытекает из связей коммутативного типа (3.8).

Обозначим через $\mathcal{N} \subset \mathcal{N}^{\#}$ подмногообразие в пространстве (3.5), заданное совместно всеми уравнениями связей (3.6), (3.8), (3.9).

Для каждого неминимального резонансного вектора $\sigma$ определим функцию

$$
A_{\sigma}= \begin{cases}1, & \text { если } \sigma=0, \\ \prod_{\varkappa \in \mathcal{M}_{\sigma}} A_{\varkappa}^{n_{\varkappa}}, & \text { если } \sigma \neq 0,\end{cases}
$$

на подмногообразии $\mathcal{N}$; здесь подмножества $\mathcal{M}_{\sigma}$ и числа $n_{\varkappa}^{\sigma}$ определены согласно (2.2). В силу связей коммутативного типа эта функция не зависит от выбора разложения (2.2) элемента $\sigma$ по минимальным.

Теперь зададим скобки на $\mathcal{N}$, следуя [10]:

$$
\left\{S_{j}, S_{k}\right\}=0, \quad\left\{S_{j}, A_{\rho}\right\}=i \rho_{j} A_{\rho}, \quad\left\{A_{\rho}, A_{\sigma}\right\}=-i f_{\rho, \sigma}(S) A_{\rho+\sigma},
$$

где $j, k=1, \ldots, n$, векторы $\rho, \sigma$ принадлежат $\mathcal{M}$, а полиномы $f_{\rho, \sigma}$ на $\mathbb{R}^{n}$ определяются следующим образом:

$$
f_{\rho, \sigma}(s) \stackrel{\text { def }}{=} \frac{[\rho, \sigma]}{s} s^{\rho+\circ}, \quad s \in \mathbb{R}^{n} .
$$

В $(3.12)$ коммутатор $[\rho, \sigma]$ и аномалия $\rho \stackrel{\circ}{+} \sigma$ заданы формулами $(3.2)$, а дробь вида $\frac{\gamma}{s}$ использована для краткого обозначения суммы:

$$
\frac{\gamma}{s} \equiv \sum_{j=1}^{n} \frac{\gamma_{j}}{s_{j}}
$$

Отметим, что в силу (3.4) функции (3.12) не имеют особенности при $s=0$.

Напомним, что функция на пуассоновом многообразии называется функцией Казимира, если она находится в инволюции со всеми функциями. Мы будем называть функцию квазиказимиром, если на своей нулевой поверхности уровня она находится в инволюции со всеми функциями. Очевидно, нулевая поверхность уровня квазиказимира является пуассоновым подмногообразием в исходном пуассоновом многообразии.

ТЕОРема 3.1. Формуль (3.11) задают скобки Пуассона на многообразии $\mathcal{N}$. Функиия

$$
C \stackrel{\text { def }}{=}\langle f, S\rangle
$$

является функиией Казимира на $\mathcal{N}$. Для каждого минимального вектора $\rho$ функиия

$$
C_{\rho} \stackrel{\text { def }}{=} A_{\rho} A_{-\rho}-S^{2 \rho}
$$

является квазиказимиром на $\mathcal{N}$; точнее, имеют место следующие соотношения:

$$
\begin{aligned}
\left\{C_{\rho}, S_{j}\right\} & =\left\{C_{\rho}, A_{\rho}\right\}=\left\{C_{\rho}, A_{-\rho}\right\}=0, \\
\left\{C_{\rho}, A_{\sigma}\right\} & =2 i \frac{\sigma \circ \rho}{S} A_{\sigma} C_{\rho}, \quad \sigma \neq \pm \rho .
\end{aligned}
$$


Здесъ $\stackrel{\circ}{\rho}$ и $\sigma \circ \rho$ определены согласно (3.2). В частности, в случае $n=2$ квазиказимир (3.15) является функиией Казимира для скобки (3.11).

ОПРЕДЕЛЕНИЕ 3.1. Подмногообразие $\mathcal{N}_{0} \subset \mathcal{N}$, заданное как совместная нулевая поверхность уровня квазиказимиров:

$$
\mathcal{N}_{0}=\left.\mathcal{N}\right|_{\left\{C_{\rho}=0 \mid \rho \in \mathcal{M}\right\}},
$$

назовем резонансным пуассоновым многообразием.

ГипотезА 3.1. Резонансное пуассоново многообразие $\mathcal{N}_{0}$ совпадает с $\mathcal{N}$ при $n \geqslant 3$.

Отметим, что на резонансном многообразии в силу связей коммутативного и некоммутативного типов имеют место соотношения

$$
A_{\rho} A_{\sigma}=S^{\rho \stackrel{\circ}{+}} A_{\rho+\sigma}
$$

для любых минимальных векторов $\rho, \sigma$.

ПРЕДЛОЖЕНИЕ 3.2. На резонансном многообразии $\mathcal{N}_{0}$ для любых резонансных (не обязательно минимальных) векторов $\rho, \sigma$ выполнены соотношения (3.16).

ДокАЗАТЕЛЬСтво аналогично доказательству предложения 11.1, которое мы приведем в $\S 11$ при рассмотрении квантового случая.

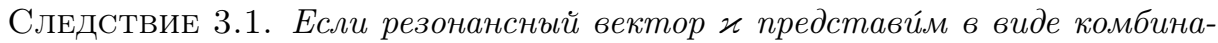
иии резонансных векторов $\varkappa^{(j)}$, m.е.

$$
\varkappa=k_{1} \varkappa^{(1)}+\cdots+k_{l} \varkappa^{(l)}, \quad k_{j} \in \mathbb{N},
$$

то на резонансном многообразии $\mathcal{N}_{0}$ выполнено соотношение

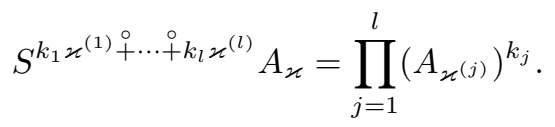

Здесь использована l-позиционная аномалия, которая определена следующим образом:

$$
\alpha_{1} \stackrel{\circ}{+} \cdots \stackrel{\circ}{+} \alpha_{l} \stackrel{\text { def }}{=} \sum_{m=1}^{l-1}\left(\left(\alpha_{1}+\cdots+\alpha_{m}\right) \stackrel{\circ}{+} \alpha_{m+1}\right)=\sum_{m=1}^{l-1}\left(\left(\alpha_{l}+\cdots+\alpha_{m+1}\right) \stackrel{\circ}{+} \alpha_{m}\right) .
$$

В правой части (3.18) участвует 2-позичионная аномалия $\stackrel{\circ}{+}$, введенная в списке операщий (3.2).

Далее, построим отображение $\mathbb{R}^{2 n} \rightarrow \mathcal{N}^{\#}$, порожденное симметриями (1.4) резонансного осциллятора:

$$
\begin{aligned}
A_{\sigma}(q, p) & =\bar{z}^{\sigma_{+}} z^{\sigma_{-}}, \quad \sigma \in \mathcal{M}, \\
S_{j}(q, p) & =\bar{z}_{j} z_{j}, \quad j=1, \ldots, n .
\end{aligned}
$$

Здесь $z_{j}$ - комплексные координаты на $\mathbb{R}^{2 n}$, заданные формулами

$$
z_{j} \stackrel{\text { def }}{=} \sqrt{\frac{f_{j}}{2}} q_{j}+\frac{i}{\sqrt{2 f_{j}}} p_{j},
$$

где $q, p$ - декартовы координаты в $\mathbb{R}^{2 n}=\mathbb{R}_{q}^{n} \times \mathbb{R}_{p}^{n}$. 
Лемма 3.2. Образ симметрийного отображения $(3.19),(3.20),(q, p) \rightarrow$ $(A, S)$, совпадает с той частъю $\mathcal{N}_{0}^{+}$резонансного многообразия $\mathcal{N}_{0}$, на которой

$$
S_{1} \geqslant 0, \ldots, S_{n} \geqslant 0 .
$$

Размерность резонансного многообразия $\mathcal{N}_{0}$ равна $2 n-1$.

ДокАзАтельство. Покажем, что функции (3.19), (3.20) удовлетворяют уравнениям связей. Связи эрмитова типа вытекают из очевидного свойства

$$
(-\rho)_{ \pm}=\rho_{\mp} \quad \forall \rho \in \mathbb{Z}^{n} .
$$

Связи некоммутативного типа вытекают из равенства

$$
(\rho+\sigma)_{ \pm}=\rho_{ \pm}+\sigma_{ \pm}-(\rho \stackrel{\circ}{+} \sigma)
$$

которое справедливо для любых $\rho, \sigma \in \mathbb{Z}^{n}$. В частности, если векторы $\rho, \sigma$ коммутируют, то $\rho \stackrel{\circ}{+} \sigma=0$, и тогда мы имеем

$$
(\rho+\sigma)_{ \pm}=\rho_{ \pm}+\sigma_{ \pm} .
$$

Отсюда вытекают связи коммутативного типа для функций (3.19).

Далее, из соотношений (3.17) вне точек, где $S_{j}=0$, любую функцию $A_{\sigma},(3.19)$, можно выразить через независимые функции $A_{\rho^{(1)}}, \ldots, A_{\rho^{(n-1)}}$, $S_{1}, \ldots, S_{n}$ (см. далее замечание 4.1). Поэтому $\operatorname{dim} \mathcal{N}_{0}=2 n-1$.

ПРЕДЛОЖЕНИЕ 3.3. Подмногообразие $\mathcal{N}_{0}^{+} \subset \mathcal{N}_{0}$, заданное неравенствами (3.22), является пуассоновым. Симметрийное отображение (3.19), (3.20) из $\mathbb{R}^{2 n}$ в $\mathcal{N}_{0}^{+}$пуассоново, т.е. переводит каноническую скобку на $\mathbb{R}^{2 n}=$ $\mathbb{R}_{q}^{n} \times \mathbb{R}_{p}^{n}$ в скобку (3.11). Функиии Казимира $C$, (3.14), при этом отображении соответствует гамильтониан осииллятора $H,(3.1)$.

ДокАЗАТЕЛьСтво повторяет доказательство теоремы 2.5 из [10].

\section{§ 4. Частичная комплексная структура на резонансном многообразии}

Покажем, как ввести на резонансном многообразии $\mathcal{N}_{0}$ частичную комплексную структуру, которая порождена симметрийным отображением (3.19), (3.20) и согласована со скобками Пуассона (3.11).

Каждый минимальный вектор $\varkappa \in \mathcal{M}$ и вектор комплексных координат $z=$ $\left(z_{1}, \ldots, z_{n}\right),(3.21)$, задают функцию $z^{\varkappa}$ на $\mathbb{R}^{2 n}$. Из (3.19), (3.20) следует, что

$$
z^{\varkappa}=\frac{A_{-\varkappa}(q, p)}{S(q, p)^{\varkappa_{-}}} .
$$

Таким образом, при симметрийном отображении (3.19), (3.20) функции $z^{\varkappa}$ на $\mathbb{R}^{2 n}$ соответствует функция

$$
W_{\varkappa} \stackrel{\text { def }}{=} \frac{A_{-\varkappa}}{S^{\varkappa}}
$$

на резонансном многообразии $\mathcal{N}_{0}$. Функция (4.1) гладкая везде, кроме некоторого подмножества границы $\partial \mathcal{N}_{0}^{+}$, где возможны особенности за счет зануления координат $S_{j}$. 
Произвольный резонансный вектор $\sigma$ можно разложить по минимальным согласно (2.2) и определить

$$
W_{\sigma} \stackrel{\text { def }}{=} \prod_{\varkappa \in \mathcal{M}_{\sigma}}\left(W_{\varkappa}\right)^{n_{\varkappa}^{\sigma}}
$$

где функция $W_{\varkappa}$ для минимального $\varkappa$ задана в (4.1). В частности, $W_{0} \equiv 1$.

Лемма 4.1. Функиия $W_{\sigma},(4.2)$, на резонансном многообразии $\mathcal{N}_{0}$ не завиcuт от выбора представления (2.2) вектора $\sigma \in \mathcal{R}$ в виде комбинации минимальных векторов. Для любых $\rho, \sigma \in \mathcal{R}$ имеет место тождество

$$
W_{\rho+\sigma}=W_{\rho} W_{\sigma} .
$$

ДокАЗАТЕЛЬСтво следует из соотношений (3.8), (3.16).

Теперь покажем, как строятся локальные комплексные координаты на $\mathcal{N}_{0}$.

ОПРЕДЕЛЕНИЕ 4.1. Набор линейно независимых векторов $\rho^{(1)}, \ldots, \rho^{(n-1)} \in$ $\mathcal{M}$ назовем резонансным базисом, если для любого резонансного вектора $\sigma \in \mathcal{R}$ коэффициенты его разложения по векторам $\rho^{(k)}-$ это целые числа:

$$
\sigma=\sum_{k=1}^{n-1} N_{\sigma}^{(k)} \rho^{(k)}, \quad N_{\sigma}^{(k)} \in \mathbb{Z} .
$$

Лемма 4.2. Резонансный базис существует.

(Конкретный вид резонансных базисов см. далее в примерах 4.1, 5.2 и в замечании 6.4.)

ЗАмЕчАНИЕ 4.1. Если вектор $\sigma$ минимальный, то из формулы (4.3) и связей типа (3.17) следует тождество

$$
S^{N_{\sigma}^{(1)} \rho^{(1)} \stackrel{\circ}{+} \cdots \stackrel{\circ}{+} N_{\sigma}^{(n-1)} \rho^{(n-1)}} A_{\sigma}=\prod_{j=1}^{n-1}\left(A_{\rho^{(j)}}\right)^{N_{\sigma}^{(j)}} .
$$

С каждым резонансным базисом можно связать комплексные координаты $w_{k}$, определенные с помощью (4.1):

$$
w_{k} \stackrel{\text { def }}{=} W_{\rho^{(k)}}, \quad k=1, \ldots, n-1 .
$$

Как следует из замечания 4.1, функции (4.2) выражаются через эти координаты мероморфно:

$$
W_{\sigma}=w^{N_{\sigma}} \quad\left(W_{\sigma}=\prod_{k=1}^{n-1}\left(w_{k}\right)^{N_{\sigma}^{(k)}}\right) .
$$

При переходе от резонансного базиса $\left\{\rho^{(j)}\right\}$ к другому резонансному базису $\left\{\widetilde{\rho}^{(j)}\right\}$ изменение $w \rightarrow \widetilde{w}$ координаты (4.4) происходит по степенному закону:

$$
\widetilde{w}=w^{\mathfrak{N}} \quad\left(\widetilde{w}_{j}=\prod_{k=1}^{n-1}\left(w_{k}\right)^{\mathfrak{N}_{k}^{j}}\right) .
$$


Здесь целочисленная матрица $\mathfrak{N}$ задается разложением базисных векторов $\widetilde{\rho}^{(j)}$ по базисным векторам $\rho^{(k)}$ :

$$
\widetilde{\rho}^{(j)}=\sum_{k=1}^{n-1} \mathfrak{N}_{k}^{j} \rho^{(k)} .
$$

Отметим, что обратная матрица $\mathfrak{N}^{-1}$ также имеет целые коэффициенты.

Теперь все многообразие $\mathcal{N}_{0}$, за исключением точки 0 (где $S=0$ и $A_{\sigma}=0$ для любого $\sigma$ ), мы покроем картами и каждой карте соотнесем резонансный базис так, чтобы все координатные функции $w_{1}, \ldots, w_{n-1},(4.4)$, отвечающие этому базису, не имели особенностей везде в данной карте.

ПримеР 4.1. В случае трехчастотного резонанса $f_{1}: f_{2}: f_{3}$ рассмотрим три резонансных базиса.

Первый базис состоит из двух (коммутирующих) векторов резонансной решетки:

$$
\left(-f_{2}, f_{1}, 0\right), \quad(\mu, \nu, 1),
$$

где $\mu, \nu$ - решение диофантова уравнения (2.3) с условием (2.4). Эта пара векторов имеет отрицательной только первую компоненту. Поэтому соответствующие данному резонансному базису комплексные координаты, определенные с помощью (4.4), (4.1), не имеют особенностей при $S_{1} \neq 0$.

Второй базис получается из (4.8) циклической перенумерацией частот $f_{1}$ : $f_{2}: f_{3} \rightarrow f_{2}: f_{3}: f_{1}$ и перестановкой компонент $(1,2,3) \rightarrow(3,1,2)$ резонансных векторов. Комплексные координаты, отвечающие этому базису, не имеют особенностей при $S_{2} \neq 0$.

Третий базис получается еще одной циклической перестановкой. Комплексные координаты здесь не имеют особенностей при $S_{3} \neq 0$.

Эти три базиса задают локальные карты (и комплексные координаты в них), покрывающие все резонансное многообразие, за исключением точки 0.

Для общего многочастотного резонанса мы получаем следующий результат.

ТеОрема 4.1. (а) Всевозможные резонансные базисы $\left\{\rho^{(j)}\right\}$ порождают атлас карт на резонансном многообразии $\mathcal{N}_{0} \backslash\{0\}$ и определяют частичную комплексную структуру типа ${ }^{3}(n-1,1)$. Общей для всех карт вещественной координатой служит функиия Казимира $C$, (3.14), а локальные комплексные координаты задаются формулой (4.4). При переходе из карты в карту комплексные координаты изменяются голоморфно согласно (4.6).

(b) Формуль (4.1), (4.2) задают представление $\sigma \rightarrow W_{\sigma}$ резонансной решет$\kappa и \mathcal{R}$ в пространстве мероморфных функций на резонансном многообразии $\mathcal{N}_{0}$. При комплексном сопряжении функиии $W_{\sigma}$ изменяются следующим образом:

$$
\overline{W_{\sigma}}=S^{\sigma} W_{-\sigma}, \quad\left|W_{\sigma}\right|^{2}=S^{\sigma} .
$$

(с) Частичная комплексная структура на $\mathcal{N}_{0} \backslash\{0\}$ согласована с пуассоновой структурой (3.11) в смысле [31]. Мероморфные функиии (4.1), (4.2) находятся в инволющии друг с другом:

$$
\left\{W_{\rho}, W_{\sigma}\right\}=0 .
$$

\footnotetext{
${ }^{3}$ Модельным пространством карты служит $\mathbb{C}^{n-1} \times \mathbb{R}^{1}$ с $(n-1)$-й комплексной координатой и одной вещественной координатой (см. [31]).
} 
Кроме того,

$$
\left\{W_{\rho}, \overline{W_{\sigma}}\right\}=i \frac{\rho \cdot \sigma}{S} W_{\rho} \overline{W_{\sigma}},
$$

где использованы обозначения (3.13).

ДокАЗАТЕЛЬСтво. В доказательстве здесь нуждаются только последние две формулы. Формула (4.10) вытекает из (3.11), (3.16) и свойства коммутатора на решетке $\mathbb{Z}^{n}$ :

$$
[-\rho,-\sigma]=-[\rho, \sigma]
$$

Аналогично, из (3.11), (3.16) и тождества на $\mathbb{Z}^{n}$

$$
[-\rho, \sigma]+\rho_{-} \cdot \sigma+\rho \cdot \sigma_{-}=\rho \cdot \sigma
$$

следует (4.11). Теорема доказана.

\section{§5. Симплектические листы и резонансные координаты Дарбу}

Введем на $\mathcal{N}_{0}$ угловые координатъ $\Phi_{\sigma}$ по формуле

$$
A_{\sigma}=S^{\circ} \exp \left\{i \Phi_{\sigma}\right\} \quad \text { или } \quad W_{\sigma}=\left|W_{\sigma}\right| \exp \left\{-i \Phi_{\sigma}\right\} .
$$

Здесь использовано обозначение $\stackrel{\circ}{\sigma}$, введенное в $(3.2)$, и учтены уравнения связей (3.6), (3.16):

$$
\left|A_{\sigma}\right|=S^{\circ}
$$

ЛЕмма 5.1. Угловые координаты находятся в инволюиии друг с другом:

$$
\left\{\Phi_{\sigma}, \Phi_{\rho}\right\}=0 .
$$

Кроме того,

$$
\left\{A_{\sigma}, \Phi_{\rho}\right\}=\frac{\rho \circ \sigma}{S} A_{\sigma}, \quad\left\{S_{j}, \Phi_{\rho}\right\}=\rho_{j},
$$

где операчия $\rho \circ \sigma$ определена в (3.2).

ДокАзАТЕЛЬСтво. Из (3.11), (3.12) и (3.3) вытекают соотношения

$$
\left\{e^{i \Phi_{\sigma}}, e^{i \Phi_{\rho}}\right\}=0, \quad\left\{A_{\sigma}, e^{i \Phi_{\rho}}\right\}=i \frac{\rho \circ \sigma}{S} A_{\sigma} e^{i \Phi_{\rho}}, \quad\left\{S_{j}, e^{i \Phi_{\rho}}\right\}=i \rho_{j} e^{i \Phi_{\rho}},
$$

а из них следуют (5.2), (5.3). Лемма доказана.

Пусть теперь $\{S=s(C)\}$ - некоторая кривая в пространстве $\mathbb{R}^{n}$, параметризованная координатой Казимира (3.14). Представим координаты $S$ на резонансном многообразии в виде

$$
S=s(C)+\sum_{k=1}^{n-1} x^{(k)} \rho^{(k)},
$$

где $\left\{\rho^{(k)}\right\}$ - некоторый резонансный базис, $x^{(k)} \in \mathbb{R}$. Таким образом, $x^{(1)}, \ldots$ $\ldots, x^{(n-1)}-$ это новые координатные функции на резонансном многообразии относительно семейства "начальных точек" $s(C)$ и базиса $\left\{\rho^{(k)}\right\}$. 
Очевидно, эти функции находятся в инволюции друг с другом, а из равенств (5.3) следует, что их скобки с угловыми координатами равны константам:

$$
\left\{x^{(j)}, x^{(k)}\right\}=0, \quad\left\{x^{(k)}, \Phi_{\sigma}\right\}=N_{\sigma}^{(k)}
$$

(числа $N_{\sigma}^{(k)}$ заданы в $(4.3)$ ). В частности, если ввести специальные угловые координаты

$$
\varphi_{k} \stackrel{\text { def }}{=} \Phi_{\rho^{(k)}}, \quad k=1, \ldots, n-1,
$$

отвечающие данному резонансному базису, то из (5.2), (5.3) получаем канонические скобки:

$$
\left\{x^{(j)}, x^{(k)}\right\}=0, \quad\left\{\varphi_{j}, \varphi_{k}\right\}=0, \quad\left\{x^{(j)}, \varphi_{k}\right\}=\delta_{j, k} .
$$

Таким образом, функции $x^{(j)}, \varphi_{j}, j=1, \ldots, n-1$, и функция Казимира $C$ задают координаты Дарбу всюду на резонансном многообразии $\mathcal{N}_{0}$, кроме подмногообразия особенностей $\partial \mathcal{N}_{0}^{+}$.

Рассмотрим теперь симплектические листы в $\mathcal{N}_{0}$ (т. е. поверхности, на которых скобка (3.11) невырождена). Все листы лежат на подмногообразиях $\{C=$ const $\}$. Скобка Пуассона на листах порождается скобкой (3.11).

Лемма 5.2. Симплектические листы в резонансном многообразии $\mathcal{N}_{0}$ имеют максимальную размерность $2 n-2$ (напомним, что $\left.\operatorname{dim} \mathcal{N}_{0}=2 n-1\right)$. В области $\mathcal{N}_{0}^{+}$замыкание всех листов компактно, однако если хотя бы одна из частот $f_{j}$ больше 1 , то лист может не совпадать со своим замыканием. Все вырожденные листы лежат на границе $\partial \mathcal{N}_{0}^{+}$.

ПримеР 5.1. В трехчастотном случае $(n=3)$ резонансное многообразие имеет размерность $\operatorname{dim} \mathcal{N}_{0}=5$. Если все частоты $f_{j}, j=1,2,3$, равны 1 , то имеется только один нульмерный лист: точка 0 , а остальные симплектические листы четырехмерны и совпадают со своим замыканием. Если какая-то частота $f_{j}$ больше 1 , то все точки вида

$$
\left\{A_{\rho}=0, S_{k}=S_{l}=0\right\} \in \mathcal{N}_{0}
$$

(где набор $j, k, l$ - это перестановка чисел $1,2,3$ ) являются нульмерными листами. При этом листы $\Omega$ максимальной размерности 4 получаются из своего замыкания $\bar{\Omega}$ путем удаления точек вида (5.7), отвечающих неединичным частотам.

В общем многочастотном случае все введенные выше функции на $\mathcal{N}_{0}$, в частности комплексные координаты $w_{j}$ и канонические координаты $x^{(j)}, \varphi_{j}$, можно сузить на симплектические листы максимальной размерности.

Теорема 5.1. Симплектические листы $\Omega \subset \mathcal{N}_{0}^{+} \cap\{C=$ const $\}$ максимальной размерности в резонансном многообразии $\mathcal{N}_{0}$ являются кэлеровыми многообразиями относительно комплексной структуры, индуцированной из $\mathcal{N}_{0}$. Симплектическая (кэлерова) форма на листах имеет вид

$$
\omega=d x \wedge d \varphi
$$

где $x, \varphi$ - координаты (5.4), (5.6), заданные каким-либо резонансным базисом $\left\{\rho^{(k)}\right\} \subset \mathcal{R}$ и начальной точкой $s(C)$. Форма (5.8) имеет особенности на множестве $\bar{\Omega} \backslash \Omega$ (которое принадлежст границе $\partial \mathcal{N}_{0}^{+}$). 
ПримеР 5.2. В трехчастотном случае рассмотрим резонансный базис

$$
\rho^{(1)}=\left(f_{2},-f_{1}, 0\right), \quad \rho^{(2)}=(\mu, \nu, 1),
$$

где $\mu, \nu$ - решение диофантова уравнения (2.3) с условием (2.4). Этот базис, в отличие от (4.8), некоммутативный: $\left[\rho^{(1)}, \rho^{(2)}\right] \neq 0$. Введем комплексные координаты, отвечающие базису (5.9), по формулам (4.4), (4.1):

$$
w_{1}=\frac{A_{-\rho^{(1)}}}{S_{2}^{f_{1}}}, \quad w_{2}=\frac{A_{-\rho^{(2)}}}{S_{1}^{|\mu|}} .
$$

Они не имеют особенностей в области

$$
S_{1} \neq 0, \quad S_{2} \neq 0 .
$$

На границе этой области лежит вершина $v=\left(0, C / f_{2}, 0\right)$ классического симплекса $\left\{S \in \mathbb{R}_{+}^{3} \mid\langle f, S\rangle=C\right\}$, которой соответствует значение комплексных переменных $w_{1}=w_{2}=0$.

В координатах (5.10) кэлерова форма на листах $\Omega$ задается формулой $\omega=$ $i \sum_{k, l=1,2}\left(\partial^{2} F / \partial \bar{w}_{k} \partial w_{l}\right) d \bar{w}_{k} \wedge d w_{l}$ через кэлеров потенциал ${ }^{4}$

$$
F=S_{1}+S_{2}+S_{3}-\frac{C}{\omega_{2}} \ln S_{2}+\text { const } .
$$

Здесь функции $S_{j}$ выражаются через $\left|w_{1}\right|,\left|w_{2}\right|$ по формулам (4.9):

$$
\left|w_{1}\right|^{2}=\frac{S_{1}^{f_{2}}}{S_{2}^{f_{1}}}, \quad\left|w_{2}\right|^{2}=\frac{S_{3} S_{2}^{\nu}}{S_{1}^{|\mu|}},
$$

с учетом соотношения (3.14). В частности, при $\left|w_{1}\right| \rightarrow 0$ получаем

$$
\begin{gathered}
S_{1} \sim\left(\frac{C}{f_{2}}\right)^{f_{1} / f_{2}}\left|w_{1}\right|^{2 / f_{2}}, \\
S_{2} \sim \frac{C}{f_{2}}-\frac{f_{1}}{f_{2}}\left(\frac{C}{f_{2}}\right)^{f_{1} / f_{2}}\left|w_{1}\right|^{2 / f_{2}}-\frac{f_{3}}{f_{2}}\left(\frac{C}{f_{2}}\right)^{f_{3} / f_{2}}\left|w_{1}\right|^{2|\mu| /\left(f_{1} f_{2}\right)}\left|w_{2}\right|^{2}, \\
S_{3} \sim\left(\frac{C}{f_{2}}\right)^{f_{3} / f_{2}}\left|w_{1}\right|^{2|\mu| / f_{2}}\left|w_{2}\right|^{2} .
\end{gathered}
$$

Хотя комплексная структура определена и в вершине $v$ классического симплекса, принадлежащей замыканию карты (5.11), вблизи этой вершины кэлеров потенциал (5.12) имеет вид

$$
F \sim \text { const }+\left(\frac{C}{f_{2}}\right)^{f_{1} / f_{2}}\left|w_{1}\right|^{2 / f_{2}}, \quad\left|w_{1}\right| \rightarrow 0, \quad\left|w_{2}\right| \rightarrow 0 .
$$

Если $f_{2} \neq 1$, то отвечающая этому потенциалу симплектическая форма

$$
\omega \sim\left(\frac{C}{f_{2}}\right)^{f_{1} / f_{2}} \frac{i d \bar{w}_{1} \wedge d w_{1}}{\left|w_{1}\right|^{2\left(f_{2}-1\right) / f_{2}}}, \quad\left|w_{1}\right| \rightarrow 0, \quad\left|w_{2}\right| \rightarrow 0,
$$

\footnotetext{
${ }^{4}$ Общая формула для потенциала в $n$-частотном случае имеет вид $F=|S|-\ln S^{v}+$ const, где $v$ - вершина классического симплекса (см. (6.1)). Эти формулы выводятся из общего определения кэлеровой формы $\omega=\sum_{k, l}\left(\left(\left\{w_{k}, \bar{w}_{l}\right\}\right)\right)^{-1} d w_{l} \wedge d \bar{w}_{k}$ с использованием (4.11).
} 
имеет (слабую) особенность в точке замыкания симплектического листа $\bar{\Omega}$, отвечающей $w=0$. (Аналогичные вычисления в двухчастотном случае см. в [14].)

Мера Лиувилля на листе $\Omega$ имеет вид

$$
\frac{1}{2}|\omega \wedge \omega|=\frac{S_{1} S_{2} S_{3}}{\left(f_{1}\right)^{2} S_{1}+\left(f_{2}\right)^{2} S_{2}+\left(f_{3}\right)^{2} S_{3}} \frac{|d \bar{w} \wedge d w|}{\left|w_{1}\right|^{2}\left|w_{2}\right|^{2}},
$$

а объем Лиувилля всего листа $\Omega$ задается формулой

$$
\int_{\Omega} \frac{|\omega \wedge \omega|}{2}=\frac{2 \pi^{2} C^{2}}{f_{1} f_{2} f_{3}}
$$

В $n$-частотном случае имеем

$$
\int_{\Omega}|\underbrace{\omega \wedge \cdots \wedge \omega}_{n-1}|=\frac{(2 \pi)^{n-1} C^{n-1}}{f_{1} \cdots f_{n}} .
$$

Формула для объема Лиувилля следует, например, из представления (5.8) и сводится к вычислению объема симплекса в $x$-пространстве, заданном неравенствами $S_{j} \geqslant 0$ (см. (5.4)).

\section{§ 6. Резонансные симплексы}

Из формул (1.1) следует, что совместный спектр квантовых операторов действия $\widehat{S}_{j}=\hat{z}_{j}^{*} \hat{z}_{j}$ в пространстве $L^{2}\left(\mathbb{R}^{n}\right)$ совпадает с решеткой $\hbar \mathbb{Z}_{+}^{n}=\{S=\hbar m \mid$ $\left.m \in \mathbb{Z}_{+}^{n}\right\}$. Эту спектральную решетку можно рассматривать как подмножество классического пространства $\mathbb{R}_{+}^{n}$, в котором принимает значения координата $S$ на резонансном многообразии $\mathcal{N}_{0}^{+}$.

Назовем классическими симплексами следующие подмножества в $\mathbb{R}_{+}^{n}$ :

$$
\mathbf{\Delta}[c] \stackrel{\text { def }}{=}\{S \mid\langle f, S\rangle=c\}, \quad c \geqslant 0 .
$$

Квантовые резонансные симплексы определим как непустые пересечения классических симплексов со спектральной решеткой $\hbar \mathbb{Z}_{+}^{n}$.

Каждому числу $M \in \mathbb{Z}_{+}$сопоставим его диофантов остов:

$$
\boldsymbol{\Delta}[M] \stackrel{\text { def }}{=}\left\{k \in \mathbb{Z}_{+}^{n} \mid\langle f, k\rangle=M\right\} .
$$

Здесь $f=\left(f_{1}, \ldots, f_{n}\right)$ - резонансный набор частот, подчиненный условиям $(\mathrm{A}),(\mathrm{B})$.

Обозначим через $d[M]$ число точек в диофантовом остове $\boldsymbol{\Delta}[M]$ и назовем это число кратностъю остова.

Лемма 6.1. Все квантовые симплексы имеют вид $\hbar \Delta[M]$ при $d[M] \geqslant 1$, причем

$$
\hbar \Delta[M] \subset \boldsymbol{\Delta}[\hbar M] .
$$

Спектр осииллятора $\widehat{H}=\langle f, \widehat{S}\rangle,(1.3)$, состоит из точек $\hbar M$, для которых $d[M] \geqslant 1$, причем кратность остова $d[M]$ совпадает с кратностью собственного значения $\hbar M$.

Таким образом, квантовый симплекс - это то подмножество в спектре действий, которое соответствует данной точке спектра резонансного осциллятора при отражении $S \rightarrow\langle f, S\rangle$. 
ЛЕмма 6.2. Если хотя бы одна из частот $f_{j}$ равна 1, mо $d[M] \geqslant 1$ при всех $M \geqslant 0$, m.е. в этом случае квантовый резонансный симплекс соответствует каждому числу $M \in \mathbb{Z}_{+}$и все остовы $\boldsymbol{\Delta}[M]$ не пусты.

В общем случае вопрос о том, при каких $M$ диофантов остов не пуст: $\boldsymbol{\Delta}[M] \neq \varnothing$, достаточно сложен.

Рассмотрим сначала двухчастотный случай $(n=2)$. Воспользуемся следующим утверждением, которое вытекает из леммы 2.1.

ЛЕмма 6.3. Любое число $M \in \mathbb{Z}_{+}$можно единственным образом представить в виде

$$
M=M_{12} f_{1} f_{2}+m_{21} f_{1}+m_{12} f_{2},
$$

где числа $M_{12}, m_{12}, m_{21}$ иелье, причем

$$
M_{12} \geqslant-1, \quad 0 \leqslant m_{12} \leqslant f_{1}-1, \quad 0 \leqslant m_{21} \leqslant f_{2}-1 .
$$

Зная число $M_{12}$ в представлении (6.3), можно вычислить кратность $d[M]$, как это было сделано в [13].

ЛЕмма 6.4. Кратность собственного значения $ћ М$ квантового осииллятора $\widehat{H}=f_{1} \widehat{S}_{1}+f_{2} \widehat{S}_{2}$ дается формулой

$$
d[M]=M_{12}+1 .
$$

Диобантов остов $\boldsymbol{\Delta}[M]$ пуст тогда и только тогда, когда $M_{12}=-1$ в представлении (6.3) числа $M$. Точка ћM принадлежит спектру $\widehat{H}$ тогда и только тогда, когда $M_{12} \geqslant 0$ в представлении (6.3). Все собственные значения вида $\hbar\left(m_{21} f_{1}+m_{12} f_{2}\right)$, где $m_{21}, m_{12}$ подчинены (6.4), и только они имеют кратность 1 .

ПримеР 6.1. Пусть $f_{1}=3, f_{2}=2$. Тогда

$$
d[1]=0, \quad d[0]=d[2]=d[3]=d[4]=d[5]=d[7]=1, \quad d[6]=2 .
$$

В общем $n$-частотном случае для каждой пары индексов $j, k \in(1, \ldots, n)$ рассмотрим представление числа $M$, аналогичное (6.3):

$$
M=M_{j k} f_{j} f_{k}+m_{k j} f_{j}+m_{j k} f_{k}, \quad \text { где } \quad 0 \leqslant m_{k j} \leqslant f_{k}-1 .
$$

Теорема 6.1. Справедлива оченка

$$
d[M] \geqslant M_{j k}+1 .
$$

Таким образом, если хотя бы для одной пары индексов $j, k$ выполнено $M_{j k} \geqslant 0$, $\operatorname{mo} d[M] \geqslant 1$, m.е. диофантов остов $\boldsymbol{\Delta}[M]$ не пуст, а число ћM принадлежит спектру осииллятора $\widehat{H},(1.3)$. Если при этом $M_{j k} \geqslant 1, \operatorname{mo} d[M] \geqslant 2$, m.е. собственное значение $ћ М$ кратное.

ДокАЗАТЕЛЬСтво. Из (6.5) следует, что число $M$ можно представить в виде $M=\langle f, k\rangle, k \in \mathbb{Z}_{+}^{n}$, выбрав

$$
k_{j}=l f_{k}+m_{k j}, \quad k_{k}=\left(M_{j k}-l\right) f_{j}+m_{j k}, \quad k_{m}=0, \quad m \neq j, k,
$$

где $l$ - любое фиксированное целое из отрезка $0 \leqslant l \leqslant M_{j k}$. Количество способов выбрать $l$ равно $M_{j k}+1$. Поэтому кратность $d[M]$ не меньше, чем $M_{j k}+1$. Теорема доказана. 
ПримеР 6.2. Если $M \geqslant\left(f_{j}-1\right)\left(f_{k}-1\right)$ для некоторой пары индексов $j, k$, то $d[M] \geqslant 1$. Это следует из теоремы 6.1 и того, что $M_{j k} \geqslant 0$ (поскольку если $M_{j k}=-1$, то $\left.M=-f_{j} f_{k}+m_{k j} f_{j}+m_{j k} f_{k}<\left(f_{j}-1\right)\left(f_{k}-1\right)\right)$.

Пример 6.3. Если в трехчастотном случае $(n=3)$ все $f_{j} \leqslant 4$, то $d[M] \geqslant 1$ для любых $M \in \mathbb{Z}_{+}$. Действительно, так как частоты $f_{j}$ взаимно простые, то хотя бы одна из них равна 1 , а далее используем лемму 6.2.

Пусть теперь $f_{1}=5, f_{2}=3, f_{3}=2$. Тогда $d[1]=0 ;$ в этом случае число $\hbar$ не принадлежит спектру осциллятора $5 \widehat{S}_{1}+3 \widehat{S}_{2}+2 \widehat{S}_{3}$, а все остальные числа $\hbar M$ $(M \geqslant 0, M \neq 1)$ принадлежат этому спектру.

Среди непустых диофантовых остовов выделяется особый случай, когда они состоят ровно из одной точки, т. е. $d[M]=1$. Этот случай тривиален.

Далее мы предполагаем, что $d[M] \geqslant 2$.

Точку $r \in \boldsymbol{\Delta}[M]$ назовем вершиной диофантова остова, если существует резонансный базис такой, что для всех $l \in \boldsymbol{\Delta}[M]$ вектор $l-r$ сонаправлен со всеми векторами базиса (т. е. все коэффициенты разложения $l-r$ по векторам базиса неотрицательны).

ЗАмЕчАНИЕ 6.1. Имеет место следующий факт: каждому резонансному базису соответствует не более одной вериины. Действительно, если бы для данного базиса $\{\rho\}$ нашлись сразу две вершины $r^{\prime}$ и $r^{\prime \prime}$, то получилось бы, что оба вектора $r^{\prime}-r^{\prime \prime}$ и $r^{\prime \prime}-r^{\prime}$ сонаправлены с базисом $\{\rho\}$. Это возможно, только если $r^{\prime}-r^{\prime \prime}=0$.

ЗАмечАнИЕ 6.2. Имеет место следующий факт: разным базисам может отвечать одна и та же вершина. Например, в случае $f=\left(1,1, f_{3}\right)$ рассмотрим два резонансных базиса:

$$
\rho^{(1)}=(1,-1,0), \quad \rho^{(2)}=\left(-f_{3}, 0,1\right) \quad \text { и } \quad \widetilde{\rho}^{(1)}=(1,-1,0), \quad \widetilde{\rho}^{(2)}=\left(0,-f_{3}, 1\right) .
$$

Первый базис некоммутативный из примера 5.2 , а второй базис коммутативный из примера 4.1. Для каждого $M \in \mathbb{Z}_{+}$получим, что точка $r=(0, M, 0)$ является вершиной остова

$$
\boldsymbol{\Delta}[M]=\left\{l \in \mathbb{Z}_{+}^{3} \mid l_{1}+l_{2}+f_{3} l_{3}=M\right\}
$$

по отношению к обоим базисам. Действительно, для каждой точки $l \in \boldsymbol{\Delta}[M]$ имеем два разложения:

$$
l=r+N^{(1)} \rho^{(1)}+N^{(2)} \rho^{(2)}=r+\widetilde{N}^{(1)} \widetilde{\rho}^{(1)}+\widetilde{N}^{(2)} \widetilde{\rho}^{(2)},
$$

с неотрицательными коэффициентами

$$
N^{(1)}=l_{1}+f_{3} l_{3}, \quad N^{(2)}=l_{3}, \quad \tilde{N}^{(1)}=l_{1}, \quad \tilde{N}^{(2)}=l_{3} .
$$

ЗАмечаниЕ 6.3. Рассмотрим трехчастотный случай. Имеет место следующий факт: если диофантов остов $\boldsymbol{\Delta}[M]$ не пуст, то в нем существует вершина (обозначим ее через $\left.r^{(2)}\right)$, отвечающая базису (5.9); координаты этой вершины задаются следующими формулами

$$
r_{3}^{(2)}=l_{3}^{*} \stackrel{\text { def }}{=} \min _{l \in \Delta[M]} l_{3}, \quad r_{1}^{(2)}=\min _{\substack{l \in \Delta[M] \\ l_{3}=l_{3}^{*}}} l_{1}, \quad r_{2}^{(2)}=\max _{\substack{l \in \Delta[M] \\ l_{3}=l_{3}^{*}}} l_{2} .
$$

\footnotetext{
${ }^{5}$ Отметим, что в силу уравнения $f_{1} l_{1}+f_{2} l_{2}=M-f_{3} l_{3}^{*}$ минимум $l_{1}$ и максимум $l_{2}$ по множеству $\left\{l \in \boldsymbol{\Delta}[M] \mid l_{3}=l_{3}^{*}\right\}$ достигаются на одном и том же векторе $l$.
} 
Для доказательства достаточно рассмотреть разложение векторов $l-r^{(2)}$ (при $l \in \boldsymbol{\Delta}[M])$ по базису (5.9):

$$
\begin{gathered}
l_{1}-r_{1}^{(2)}=N^{(1)} f_{2}+N^{(2)} \mu, \quad l_{2}-r_{2}^{(2)}=-N^{(1)} f_{1}+N^{(2)} \nu, \\
l_{3}-r_{3}^{(2)}=N^{(2)},
\end{gathered}
$$

и показать, что для каждого $l \in \boldsymbol{\Delta}[M]$ коэффициенты разложения $N^{(1)}$ и $N^{(2)}$ неотрицательны. Оценка $N^{(2)} \geqslant 0$ вытекает из третьего соотношения и формулы для $r_{3}^{(2)}$, а оценка $N^{(1)} \geqslant 0$ вытекает из первого соотношения, если учесть, что в нем $\mu<0, r_{1}^{(2)}<f_{2}$. Действительно, из предположения того, что $N^{(1)}<0$, следует

$$
l_{1}=\left(r_{1}^{(2)}+N^{(1)} f_{2}\right)+N^{(2)} \mu<0,
$$

что противоречит условию $l_{1} \geqslant 0$.

Пусть теперь $(j, k, s)$ - циклическая перестановка чисел $(1,2,3)$. Рассмотрим резонансный базис, полученный путем соответствующей циклической перестановки базиса (5.9). Тогда по отношению к этому базису следующая точка $r^{(k)}$ является вершиной остова $\boldsymbol{\Delta}[M]$ :

$$
r_{s}^{(k)}=l_{s}^{*} \stackrel{\text { def }}{=} \min _{l \in \Delta[M]} l_{s}, \quad r_{j}^{(k)}=\min _{\substack{l \in \Delta[M] \\ l_{s}=l_{s}^{*}}} l_{j}, \quad r_{k}^{(k)}=\max _{\substack{l \in \Delta[M] \\ l_{s}=l_{s}^{*}}} l_{k} .
$$

При этом если в представлении (6.5) число $M_{j k}$ не меньше нуля, то формулы (6.6а) принимают вид

$$
r_{s}^{(k)}=0, \quad r_{j}^{(k)}=m_{k j}, \quad r_{k}^{(k)}=M_{j k} f_{j}+m_{j k}
$$

ЗАмЕчАнИЕ 6.4. Формула (6.6b) легко обобщается на $n$-частотный случай. Пусть в представлении (6.3) число $M_{12}$ неотрицательное. Тогда точка

$$
r^{(2)}=(m_{21}, M_{12} f_{1}+m_{12}, \underbrace{0, \ldots, 0}_{n-2})
$$

является вершиной диофантова остова $\boldsymbol{\Delta}[M]$, а в качестве соответствующего резонансного базиса можно взять базис

$$
\begin{gathered}
\rho^{(1)}=\left(f_{2},-f_{1}, 0,0, \ldots, 0,0\right), \\
\rho^{(2)}=\left(\mu_{3}, \nu_{3}, 1,0, \ldots, 0,0\right), \\
\ldots \ldots \ldots \ldots \ldots \ldots \ldots \ldots . . \ldots, 1) .
\end{gathered}
$$

Это $n$-мерный аналог базиса (5.9). В данных формулах $\mu_{l}, \nu_{l}$ - это решение диофантова уравнения

$$
\mu_{l} f_{1}+\nu_{l} f_{2}+f_{l}=0
$$

подчиненное условию $0 \leqslant \nu_{l} \leqslant f_{1}-1$ (здесь $l=3, \ldots, n$ ).

Взяв другую пару индексов $j, k$, для которых $M_{j k} \geqslant 0$ в представлении (6.5), можно аналогично предъявить другие вершины $r^{(k)}$ остова $\boldsymbol{\Delta}[M]$.

Отметим, что каждой вершине $r \in \boldsymbol{\Delta}[M]$ соответствует точка $\hbar r$ в квантовом симплексе; назовем ее верииной квантового симплекса. 
Лемма 6.5. При $\hbar \rightarrow 0$ и $M \sim \hbar^{-1}$ вершинь квантового симплекса $\hbar \Delta[M]$ близки к вершинам классического симплекса $\mathbf{\Delta}[\hbar M]$.

ДокАЗАТЕЛЬСтво. При большом $M$ все числа $M_{j k}$ в представлениях (6.5) также велики (и заведомо положительны). Вершина квантового симплекса, сопоставленная каждой паре индексов $j, k$ по формуле вида (6.7), где $j=1$, $k=2$, отличается от вершины вида $\left(0, \frac{\hbar M}{f_{2}}, 0, \ldots, 0\right)$ классического симплекса на вектор

$$
\left(\hbar m_{21},-\frac{\hbar m_{21} f_{1}}{f_{2}}, 0, \ldots, 0\right),
$$

который стремится к нулю при $\hbar \rightarrow 0$. Таким образом, для вершин вида (6.7) утверждение леммы 6.5 выполнено, а остальные вершины квантового симплекса $\hbar \boldsymbol{\Delta}[M]$ при $M \sim \hbar^{-1} \rightarrow \infty$ расположены ближе к вершинам классического симплекса $\boldsymbol{\Delta}[\hbar M]$, чем вершины вида (6.7); поэтому для них утверждение также выполнено.

\section{§ 7. Квантовые листы и пространства голоморфных сечений}

Пару $R=(r,\{\rho\})$, состоящую из вершины $r$ и отвечающего ей резонансного базиса $\{\rho\}$, мы назовем репером диофантового остова $\boldsymbol{\Delta}[M]$.

Каждому реперу сопоставим пространство $\mathbb{C}^{n-1}$ как модельное пространство локальной карты. Пусть заданы две карты, занумерованные реперами $R=(r,\{\rho\})$ и $\widetilde{R}=(\widetilde{r},\{\widetilde{\rho}\})$. Комплексные координаты $w$ и $\widetilde{w}$ в них определяются резонансными базисами $\{\rho\}$ и $\{\widetilde{\rho}\}$ по формуле (4.4). Склейка карт осуществляется по формуле (4.6), в которой $\mathfrak{N}$ - целочисленная матрица перехода (4.7) от базиса $\{\rho\}$ к базису $\{\widetilde{\rho}\}$. Результатом склейки будет некоторое комплексное многообразие. Назовем его квантовым листом и обозначим $\Omega_{\hbar}[M]$.

Напомним, что через $\Omega=\Omega[c]$ мы обозначаем симплектические листы максимальной размерности, лежащие в $\mathcal{N}_{0}^{+} \subset \mathcal{N}_{0}$ на поверхностях уровня функции Казимира $\{C=c\},(3.14)$. Конструкция комплексной структуры на $\mathcal{N}_{0}$ (см. формулы (4.1), (4.9)) показывает, что справедливо следующее утверждение.

ПРЕДЛОЖЕНИЕ 7.1. Классический симплектический лист $\Omega=\Omega[\hbar M]$ плотно вложен в квантовый лист $\Omega_{\hbar}=\Omega_{\hbar}[M]$, m.e.

$$
\Omega \subset \bar{\Omega} \approx \Omega_{\hbar}
$$

Это вложение сохраняет комплексную структуру.

СлЕДСТвиЕ 7.1. Квантовые листы $\Omega_{\hbar}$ компактны.

Отметим, что классическую кэлерову форму $\omega$ нельзя расширить с классических листов на квантовые листы при наличии неединичных частот (это видно, например, из (5.14)). Мы покажем, как задать квантовую кэлерову форму на квантовых листах с помощью групповой структуры резонансной решетки, следуя общей идеологии из [31], [32]. Однако сначала введем пространство голоморфных сечений над квантовым листом.

Построим вначале пучок ростков голоморфных функций над квантовым листом и определим гильбертово пространство сечений этого пучка. 
На квантовом листе $\Omega_{\hbar}=\Omega_{\hbar}[M]$ в карте с номером $R$ рассмотрим семейство функций $U_{R}^{t}$, заданных формулой

$$
U_{R}^{t} \stackrel{\text { def }}{=} \sqrt{\frac{\hbar^{|r|} r !}{\hbar^{|t|} t !}} W_{t-r}, \quad t \in \Delta[M] .
$$

Здесь через $r$ обозначена вершина репера $R$, а мероморфные функции $W_{\sigma}$ определены формулой (4.5) через комплексные координаты в карте с номером $R$. В данных координатах эти функции являются мономами, поскольку $r$ - вершина остова $\boldsymbol{\Delta}[M]$.

Если точка $t$ в (7.1) совпадает с какой-то вершиной остова $\boldsymbol{\Delta}[M]$, то мы будем также использовать другое обозначение:

$$
V_{R}^{T} \stackrel{\text { def }}{=} U_{R}^{t}
$$

где $T$ - любой репер с вершиной $t$. Таким образом, для разных реперов $T$ с одной и той же вершиной $t$ функция $V_{R}^{T}$ одна и та же. Очевидно, что $V_{R}^{R} \equiv 1$.

В силу группового свойства отображения $\rho \rightarrow W_{\rho}$ (лемма 4.1) при любом $t \in \boldsymbol{\Delta}[M]$ на пересечении карт с номерами $R$ и $Q$ выполнено соотношение

$$
U_{Q}^{t}=V_{Q}^{R} U_{R}^{t}
$$

В частности, для любых трех реперов $R, L, Q$ на пересечении соответствующих трех карт имеем тождество

$$
V_{Q}^{L}=V_{Q}^{R} V_{R}^{L}
$$

Свойство (7.4) означает, что набор голоморфных функций $\left\{V_{Q}^{R}\right\}$, заданных на пересечениях карт квантового листа $\Omega_{\hbar}$, можно рассматривать как функиии склейки пучка $\left(\Omega_{\hbar}\right)$ ростков голоморфных функций на $\Omega_{\hbar}$.

При этом формула (7.3) показывает, что для любого фиксированного $t \in$ $\boldsymbol{\Delta}[M]$ набор функций $U^{t}=\left\{U_{R}^{t}\right\}$ задает сечение пучка П $\left(\Omega_{\hbar}\right)$. Таким образом, получаем следующий результат.

Лемма 7.1. Точкам $t$ диофантова остова $\boldsymbol{\Delta}[M]$ соответствуют сечения $U^{t}$ пучка $\Pi\left(\Omega_{\hbar}\right)$ ростков голоморфных функций на квантовом листе $\Omega_{\hbar}$. Эти сечения линейно независимы.

Введем теперь гильбертову структуру в пространстве сечений пучка П $\left(\Omega_{\hbar}\right)$ так, чтобы набор сечений $\left\{U^{t} \mid t \in \boldsymbol{\Delta}[M]\right\}$ был ортонормированным:

$$
\left(U^{k}, U^{t}\right)=\delta_{k, t} \text {. }
$$

Тем самым, мы построили некоторое каноническое гильбертово пространство $\mathcal{L}\left(\Omega_{\hbar}\right)$ голоморфных сечений над квантовым листом $\Omega_{\hbar}$, порожденное групповой структурой резонансной решетки согласно (7.1) и лемме 4.1.

Вид числовых коэффициентов в (7.1) назовем фоковским. Он имитирует вид коэффициентов обычного нормированного базиса Фока в пространстве голоморфных функций на евклидовом пространстве [36].

Обозначим через $\mathcal{K}$ воспроизводящее ядро гильбертова пространства $\mathcal{L}\left(\Omega_{\hbar}\right)$. Оно определяется как сечение пучка $\left(\Pi^{*} \times \Pi\right)\left(\Omega_{\hbar}\right)$, которое в карте с номером $R$ задано формулой

$$
\mathcal{K}_{R} \stackrel{\text { def }}{=} \sum_{t}\left|\psi_{R}^{t}\right|^{2}
$$


Здесь суммирование ведется по любому ортонормированному базису сечений $\left\{\psi^{t}\right\}$ пучка П $\left(\Omega_{\hbar}\right)$. Хорошо известно [37], что сечение (7.6) не зависит от выбора базиса. Поэтому, выбирая в качестве базиса набор сечений $\left\{U^{t}\right\}$, получаем следующую формулу для воспроизводящего ядра в карте с номером $R$ :

$$
\mathcal{K}_{R}=\sum_{t \in \Delta[M]}\left|U_{R}^{t}\right|^{2} .
$$

Остается подставить сюда явные выражения (7.1), (4.9).

Теорема 7.1. Пусть число $M \in \mathbb{Z}_{+}$таково, что $d[M] \geqslant 1$, т.е. диобантов остов $\boldsymbol{\Delta}[M]$ не пуст, и пусть полином $\mathcal{P}^{[M]}$ на $\mathbb{R}^{n-1}$ задан формулой

$$
\mathcal{P}^{[M]}(s) \stackrel{\text { def }}{=} \sum_{\substack{\langle t, f\rangle=M \\ t \in \mathbb{Z}_{+}^{n}}} \frac{s^{t}}{\hbar^{|t|} t !} .
$$

Гилъбертово пространство голоморфных сечений $\mathcal{L}\left(\Omega_{\hbar}\right)$ над квантовым листом $\Omega_{\hbar}=\Omega_{\hbar}[M]$ имеет размерность $d[M]$. Воспроизводящее ядро этого пространства в карте с номером $R$ вычисляется через полином (7.8) следующим образом:

$$
\mathcal{K}_{R}=\frac{r ! \hbar^{|r|}}{S^{r}} \mathcal{P}^{[M]}(S)
$$

Здесъ через $r$ обозначена вершина репера $R$, а координаты $S$ перенесенын н $\Omega_{\hbar}$ с замыкания симплектического листа $\Omega[\hbar M]$ согласно (4.9), (3.14), m.е.

$$
S^{\rho}=\left|W_{\rho}\right|^{2}, \quad\langle f, S\rangle=\hbar M
$$

где $\{\rho\}-$ базис, отвечающий реперу $R$.

$B$ локальных комплексных координатах $w$ в карте $c$ номером $R$ сечение (7.9) является полиномом

$$
\mathcal{K}_{R}=\sum_{\sigma \in \mathcal{R}_{r}} \frac{r !}{\hbar^{\left|\sigma_{+}\right|-\left|\sigma_{-}\right|}(r+\sigma) !} \bar{w}^{N_{\sigma}} w^{N_{\sigma}} .
$$

Здесь подмножество $\mathcal{R}_{r} \subset \mathcal{R}$ задано условием

$$
\sigma \in \mathcal{R}_{r} \Longleftrightarrow r+\sigma \in \Delta[M]
$$

а векторы $N_{\sigma} \in \mathbb{Z}_{+}^{n-1}$ определены разложением (4.3) векторов $\sigma$ по резонансному базису из репера $R$ с верииной $r$.

\section{§ 8. Квантовая воспроизводящая мера и квантовая кэлерова структура}

Скалярное произведение в гильбертовом пространстве $\mathcal{L}\left(\Omega_{\hbar}\right)$, определенное формулой (7.5), теперь можно попытаться записать в виде интеграла по мере, следуя [31], [32]:

$$
(\varphi, \psi)=\frac{1}{(2 \pi \hbar)^{n-1}} \int_{\Omega_{\hbar}} \rho_{\psi \mid \varphi} d m_{\hbar}
$$


Здесь $\varphi, \psi$ - два произвольных сечения из $\mathcal{L}\left(\Omega_{\hbar}\right)$, их взаимная функция плотности $\rho_{\psi \mid \varphi}$ на $\Omega_{\hbar}$ задана формулой

$$
\rho_{\psi \mid \varphi} \stackrel{\text { def }}{=} \frac{\bar{\psi} \varphi}{\mathcal{K}},
$$

а воспроизводящая мера $d m_{\hbar}$ в локальных координатах $w$ в карте с номером $R$ определена равенством

$$
d m_{\hbar}=\mathcal{K}_{R} \mathcal{J}_{R} d \bar{w} d w .
$$

В этом представлении меры мы используем воспроизводящее ядро $\mathcal{K},(7.9)$, и некоторое сечение $\mathcal{J}$ пучка $\left(\Pi^{-1 *} \times \Pi^{-1}\right)\left(\Omega_{\hbar}\right)$.

ТЕОРема 8.1. СКалярное произведение в пространстве голоморфных сечений $\mathcal{L}\left(\Omega_{\hbar}\right)$ над квантовым листом $\Omega_{\hbar}$ вычисляется по формулам (8.1)-(8.3), причем сечение $\mathcal{J}$ в (8.3), в карте с номером $R$, имеет вид

$$
\mathcal{J}_{R}=\frac{S^{r-\Sigma \rho}}{r ! \hbar^{|r|}} Q^{[M]}(S) .
$$

Здесь $r$ - вершина репера $R,\{\rho\}$ - соответствующий резонансный базис, $\sum \rho=\sum_{j=1}^{n-1} \rho^{(j)}, M=\langle f, r\rangle$, а функиия $Q^{[M]}$ задана формулой

$$
Q^{[M]}(s) \stackrel{\text { def }}{=} \frac{s_{1} \cdots s_{n}}{\hbar} \int_{0}^{\infty} y^{M+|f|-1} \exp \left\{-\frac{1}{\hbar} \sum_{j=1}^{n-1} s_{j} y^{f_{j}}\right\} d y .
$$

ДокАЗАТЕЛЬство. Утверждение теоремы эквивалентно проверке "воспроизводящего свойства" для полинома $\mathcal{K}_{R},(7.6)-(7.10)$ :

$$
\frac{1}{(2 \pi \hbar)^{n-1}} \int \mathcal{K}_{R}\left(w^{\prime} \bar{w}\right) \mathcal{K}_{R}\left(w \bar{w}^{\prime \prime}\right) \mathcal{J}_{R} d w d \bar{w}=\mathcal{K}_{R}\left(w^{\prime} \bar{w}^{\prime \prime}\right)
$$

где в аргументах полинома $\mathcal{K}_{R}$ используется покомпонентное умножение точек из $\mathbb{C}^{n-1}$. Заметим, что если координаты $w$ связаны с координатами $z$ симметрийным отображением

$$
w_{j}=z^{\rho^{(j)}}, \quad j=1, \ldots, n-1,
$$

то по теореме о вычетах полином $\mathcal{K}_{R}$ можно представить в форме интеграла:

$$
\mathcal{K}_{R}\left(w^{\prime} \bar{w}^{\prime \prime}\right)=\frac{\hbar^{|r|} r !}{2 \pi\left(z^{\prime} \bar{z}^{\prime \prime}\right)^{r}} \int_{0}^{2 \pi} \exp \left\{i\langle f, r\rangle t+\frac{1}{\hbar} \sum_{j=1}^{n} z_{j}^{\prime} \bar{z}_{j}^{\prime \prime} e^{-i f_{j} t}\right\} d t .
$$

Теперь рассмотрим хорошо известную формулу Фока

$$
\frac{1}{(2 \pi \hbar)^{n}} \int_{\mathbb{C}^{n}} \exp \left\{\frac{\left\langle z^{\prime}, \bar{z}\right\rangle+\left\langle\bar{z}^{\prime \prime}, z\right\rangle-\langle\bar{z}, z\rangle}{\hbar}\right\} d z d \bar{z}=\exp \left\{\frac{\left\langle z^{\prime}, \bar{z}^{\prime \prime}\right\rangle}{\hbar}\right\},
$$

заменим в ней $z_{j}^{\prime}$ на $z_{j}^{\prime} e^{-i f_{j} t^{\prime}}, z_{j}^{\prime \prime}$ на $z_{j}^{\prime \prime} e^{-i f_{j} t^{\prime \prime}}$ и проинтегрируем по $t^{\prime}$ и $t^{\prime \prime}$ от 0 до $2 \pi$. Тогда в силу упомянутого интегрального представления для $\mathcal{K}_{R}$ получим тождество

$$
\frac{1}{(2 \pi \hbar)^{n-1}} \int_{\mathbb{C}^{n}} \mathcal{K}_{R}\left(w^{\prime} \bar{w}\right) \mathcal{K}_{R}\left(w \bar{w}^{\prime \prime}\right) \frac{\left|z^{r}\right|^{2}}{2 \pi \hbar^{|r|+1} r !} e^{-|z|^{2} / \hbar} d z d \bar{z}=\mathcal{K}_{R}\left(w^{\prime} \bar{w}^{\prime \prime}\right) .
$$


Сделаем в этом тождестве под знаком интеграла замену $w_{1}=z^{\rho^{(1)}}, \ldots, w_{n-1}=$ $z^{\rho^{(n-1)}}, w_{n}=z^{f}$. Якобиан замены равен 6

$$
\left(\langle f, f\rangle \frac{\left|w_{1} \cdots w_{n}\right|}{\left|z_{1} \cdots z_{n}\right|}\right)^{2}
$$

Тогда получим искомое "воспроизводящее свойство" для $\mathcal{K}_{R}$, в котором плотность $\mathcal{J}_{R}$ задается формулой

$$
\mathcal{J}_{R}(w \bar{w})=\frac{1}{2 \pi \hbar^{|r|+1} r !\langle f, f\rangle^{2}\left|w_{1} \cdots w_{n-1}\right|^{2}} \int_{\mathbb{C}} \frac{\left|z^{r}\right|^{2}\left|z_{1} \cdots z_{n}\right|^{2}}{\left|w_{n}\right|^{2}} e^{-\frac{|z|^{2}}{\hbar}} d w_{n} d \bar{w}_{n} .
$$

Здесь под знаком интеграла координаты $z_{j}$ выражены через $w_{1}, \ldots, w_{n-1}, w_{n}$ по формулам

$$
z_{j}=w_{1}^{p_{j}^{(1)}} \cdots w_{n}^{p_{j}^{(n)}}
$$

где $p^{(1)}, \ldots, p^{(n)}$ - векторы, составляющие обратную матрицу по отношению к матрице из векторов $\rho^{(1)}, \ldots, \rho^{(n-1)}, \rho^{(n)} \equiv f$, т. е.

$$
\sum_{l=1}^{n} p_{j}^{(l)} \rho_{k}^{(l)}=\delta_{j, k}, \quad j, k=1, \ldots, n
$$

при этом $p_{j}^{(n)}=f_{j} /\langle f, f\rangle$, а все числа $p_{j}^{(l)}, 1 \leqslant l \leqslant n-1$, целые.

Переходя в последнем интеграле к координатам $x, \varphi$ по формуле

$$
w_{n}=\sqrt{x^{\langle f, f\rangle}} e^{i \varphi}
$$

и учитывая, что полярный угол пробегает промежуток $[0,2 \pi\langle f, f\rangle]$, получим новую формулу

$$
\begin{aligned}
\mathcal{J}_{R}=\frac{1}{\hbar^{|r|+1} r !} \prod_{k=1}^{n-1}\left|w_{k}\right|^{2\left(\left\langle r, p^{(k)}\right\rangle+\left|p^{(k)}\right|-1\right)} \\
\quad \times \int_{0}^{\infty} x^{\langle f, r\rangle+|f|-1} \exp \left\{-\frac{1}{\hbar} \sum_{j=1}^{n} x^{f_{j}} \prod_{k=1}^{n-1}\left|w_{k}\right|^{2 p_{j}^{(k)}}\right\} d x .
\end{aligned}
$$

Осталось заметить, что вместо $\left|w_{j}\right|$ здесь удобнее использовать координаты $S_{1}, \ldots, S_{n}$ (см. (4.9)). После замены $x=S^{f /\langle f, f\rangle} y$ (под знаком последнего интеграла) окончательно получим (8.4), (8.5).

Из теоремы 8.1, в частности, следует, что для любого ортонормированного базиса $\left\{\psi^{t}\right\}$ в пространстве $\mathcal{L}\left(\Omega_{\hbar}\right)$ в силу (7.6) имеем

$$
\operatorname{dim} \mathcal{L}\left(\Omega_{\hbar}\right)=\sum_{t}\left\|\psi^{t}\right\|^{2}=\sum_{t} \frac{1}{(2 \pi \hbar)^{n-1}} \int_{\Omega_{\hbar}} \frac{\left|\psi^{t}\right|^{2}}{\mathcal{K}} d m_{\hbar}=\frac{1}{(2 \pi \hbar)^{n-1}} \int_{\Omega_{\hbar}} d m_{\hbar} .
$$

Поэтому из леммы 6.1 и теоремы 7.1 получаем следующий результат.

\footnotetext{
6 Здесь учтено тождество для детерминанта матрицы, составленной из векторов резонансного базиса и вектора частот: $\left|\operatorname{det}\left(\rho^{(1)} \cdots \rho^{(n-1)} f\right)\right|=\langle f, f\rangle$.
} 
СлеДСтвиЕ 8.1. Кратность собственного значения $ћ$ резонансного осииллятора (1.3) вычисляется по формуле

$$
d[M]=\frac{1}{(2 \pi \hbar)^{n-1}} \int_{\Omega_{\hbar}} d m_{\hbar} .
$$

Здесь квантовая воспроизводящая мера имеет вид

$$
d m_{\hbar}=\left(\mathcal{P}^{[M]} Q^{[M]}\right)(S) \frac{d \bar{w} d w}{S^{\Sigma \rho}},
$$

где $\mathcal{P}^{[M]}$ и $Q^{[M]}$ заданы в $(7.8),(8.5)$, через $w$ обозначены комплексные координаты в локальной карте с номером $R$ на квантовом листе $\Omega_{\hbar}[M]$, а $\rho-$ резонансный базис из репера $R$.

Теперь построим на квантовом листе $\Omega_{\hbar}$ замкнутую 2-форму $\omega_{\hbar}$, порожденную воспроизводящим ядром пространства $\mathcal{L}\left(\Omega_{\hbar}\right)$. В карте с номером $R$ и с комплексными координатами $w$ эта форма задается формулой

$$
\omega_{\hbar}=i \hbar \sum_{l, j=1}^{n-1} \frac{\partial^{2}}{\partial \bar{w}_{l} \partial w_{j}}\left(\ln \mathcal{K}_{R}\right) d \bar{w}_{l} \wedge d w_{j}
$$

От выбора карты эта форма не зависит. Мы назовем ее, следуя [31], [32], квантовой кэлеровой формой на $\Omega_{\hbar}$.

Вычислим асимптотику квантовой кэлеровой формы, а также воспроизводящей меры при $\hbar \rightarrow 0$.

Лемма 8.1. Вне окрестности точек, где хотя бъ одна координата $S_{j}$ обращается в нуль, воспроизводящее ядро $\mathcal{K}_{R},(7.9)$, и плотность $\mathcal{J}_{R},(8.4)$, имеют следующие асимптотики при $\hbar \rightarrow 0$ и $M \sim 1 / \hbar$ :

$$
\begin{gathered}
\mathcal{K}_{R}=c_{r} \frac{\exp \{|S| / \hbar\}}{S^{r}}(\varkappa(S)+O(\hbar)), \\
\mathcal{J}_{R}=\hbar^{-|r|-1 / 2} \frac{\sqrt{2 \pi}}{r !} S^{r} \exp \left\{-\frac{|S|}{\hbar}\right\}\left(\frac{S_{1} \cdots S_{n}}{S^{\rho^{(1)}+\cdots+\rho^{(n-1)}}} \varkappa(S)+O(\hbar)\right) .
\end{gathered}
$$

Здесъ $\rho$ и $r$ - резонансньй базис и вершина репера $R$ соответственно, а функ-

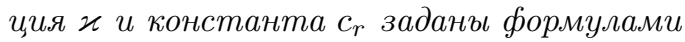

$$
\begin{gathered}
\varkappa(S)=\left(f_{1}^{2} S_{1}+\cdots+f_{n}^{2} S_{n}\right)^{-1 / 2}, \\
c_{r}=\hbar^{|r|+1 / 2} r ! e^{-|r|} \sqrt{2 \pi}\left(f_{1}^{2} r_{1}+\cdots+f_{n}^{2} r_{n}\right)^{1 / 2} \sum_{\substack{\langle t-r, f\rangle=0 \\
t \in \mathbb{Z}_{+}^{n}}} \frac{r^{t}}{t !} .
\end{gathered}
$$

ДокАЗАТЕЛЬСтво следует из системы дифференциальных уравнений, которым удовлетворяют функции $\mathcal{K}_{R}$ и $\mathcal{J}_{R}$. Эти уравнения приведены далее в замечании 13.1. Асимптотика при $\hbar \rightarrow 0$ решения этих уравнений имеет ВКБ-вид:

$$
\mathcal{K}_{R}=\exp \left\{\frac{F}{\hbar}\right\}(\varphi+O(\hbar)), \quad \mathcal{J}_{R}=\exp \left\{-\frac{F}{\hbar}\right\}(\psi+O(\hbar)),
$$

где $F$ - кэлеров потенциал классической симплектической формы $\omega$ (см. формулу (5.12)). Для амплитуд $\varphi, \psi$ получаются простые уравнения, решение которых приводит к формулам, указанным в лемме. 
Напомним (предложение 7.1), что квантовый лист $\Omega_{\hbar}=\Omega_{\hbar}[M]$ отождествляется с замыканием $\bar{\Omega}$ классического симплектического листа $\Omega=\Omega[\hbar M]$ резонансного многообразия. Из леммы 8.1 и формул $(8.7),(8.8)$ получаем следующий результат.

Теорема 8.2. В точках квантового листа $\Omega_{\hbar}$, которые соответствуют точкам классического листа $\Omega$, квантовая кэлерова форма (8.8) приблиюается классической симплектической формой (5.8), а воспроизводящая меpa (8.3) при $\hbar \rightarrow 0, M \sim 1 / \hbar$ приближается классической мерой Лиувилля $d m=\frac{1}{(n-1) !}|\underbrace{\omega \wedge \cdots \wedge \omega}_{n-1}|$, а именно

$$
\omega_{\hbar}=\omega+O(\hbar), \quad d m_{\hbar}=d m+O(\hbar) .
$$

В точках квантового листа $\Omega_{\hbar}$, которье соответствуют граничным точкам $\bar{\Omega} \backslash \Omega$ классического листа, асимптотика (8.9) нарушается; в этих точках классическая симплектическая форма $\omega$ и мера $d m$ сингулярны.

ЗАмЕчАНИЕ 8.1. Из (8.9) следует, что при достаточно малом $\hbar$ и $M \sim 1 / \hbar$ квантовая кэлерова форма $\omega_{\hbar}$ невырождена в точках классического симплектического листа. Однако она может вырождаться на его границе, оставаясь гладкой. Невырожденность формы $\omega_{\hbar}$ в точке границы, соответствующей вершине $r \in \Delta[M]$, имеет место тогда и только тогда, когда все точки, полученные из $r$ с помощью базисных векторов, попадают в квантовый симплекс, т. е.

$$
r+\rho^{(j)} \in \Delta[M], \quad j=1, \ldots, n-1 .
$$

Это следует из явной формулы для $\omega_{\hbar}$ в комплексных координатах $w$ на квантовом листе, где вершине $r$ соответствует $w=0$ :

$$
\left.\omega_{\hbar}\right|_{w=0}=i \hbar \sum_{r+\rho^{(j)} \in \Delta[M]} c_{\rho^{(j)}}^{r} d \bar{w}_{j} \wedge d w_{j}, \quad c_{\rho^{(j)}}^{r}=\frac{r !}{\hbar^{\left|\rho_{+}^{(j)}\right|-\left|\rho_{-}^{(j)}\right|}\left(r+\rho^{(j)}\right) !} .
$$

Пример, в котором квантовая форма вырождена, дает резонанс $1: 2: 3$ при $M=2$ в вершине $r=(2,0,0)$ с базисом $(4.8)$.

Отметим, что результат, аналогичный теореме 8.2 , имеет место для целого ряда других квантовых систем, в которых алгебра симметрий не является алгеброй Ли [13], [14], [31], [32]: квантовые геометрические объекты $\omega_{\hbar}, d m_{\hbar}$ определены глобально и гладко на $\Omega_{\hbar}$, в отличие от классических геометрических объектов.

ЗАмЕчАНИЕ 8.2. Асимптотические разложения (8.9) могут быть продлены до любого порядка малости по $\hbar$ :

$$
\omega_{\hbar} \simeq \omega+\sum_{k=1}^{\infty} \hbar^{k} \lambda_{k}, \quad d m_{\hbar}=d m\left(1+\sum_{k=1}^{\infty} \hbar^{k} d_{k}\right) .
$$

Здесь коэффициенты $\lambda_{k}-$ замкнутые 2-формы, а коэффициенты $d_{k}$ - гладкие функции на $\Omega$. При этом каждая $d_{k}$ задается явными формулами [32] через формы $\omega, \lambda_{1}, \ldots, \lambda_{k}$.

После подстановки (8.11) в (8.6) получаем формулу для кратности $d[M]$, в которой участвуют лишь первые $n-1$ функций $d_{k}$, причем параметр $\hbar$ можно 
положить равным 1 (см. [32]):

$$
d[M]=\frac{1}{(2 \pi)^{n-1}} \int_{\Omega[M]}\left(1+\sum_{k=1}^{n-1} d_{k}\right) \frac{1}{(n-1) !}|\omega \wedge \cdots \wedge \omega| .
$$

\section{§ 9. Когомологии квантового листа}

Поскольку квантовый лист $\Omega_{\hbar}=\Omega_{\hbar}[M]$ компактен, то все группы когомологий $H^{2 k}\left(\Omega_{\hbar}\right)$, где $k=1, \ldots, n-1$, нетривиальны. При этом класс когомологий квантовой кэлеровой формы $\frac{1}{2 \pi \hbar} \omega_{\hbar}$, заданной в $(8.8)$, обязан быть целочисленным [38].

Рассмотрим для простоты случай $n=3$.

Теорема 9.1. Нестягиваемые 2-цикль в квантовом листе $\Omega_{\hbar}[M]$ coответствуют ребрам классического резонансного симплекса $\mathbf{\Delta}[\hbar M]$, а именно если $(j, k, s)$ - чиклическал перестановка чисел $(1,2,3)$, то ребру, соединяющему $j$-ю и k-ю вериины, соответствует (положительно ориентированныи) иикл $\Sigma_{j k}=\left\{f_{j} S_{j}+f_{k} S_{k}=\hbar M\right\}$. Выполнены формуль

$$
\frac{1}{2 \pi \hbar} \int_{\Sigma_{j k}} \omega_{\hbar}=\left[\frac{r_{k}^{(k)}}{f_{j}}\right] .
$$

Здесъ $r^{(k)}$ - вериина (6.6а) диобантова остова $\boldsymbol{\Delta}[M]$, а квадратными скобками обозначена целая часть числа. Если $M_{j k} \geqslant 0$ в представлении (6.5), то формула (9.1) принимает вид

$$
\frac{1}{2 \pi \hbar} \int_{\Sigma_{j k}} \omega_{\hbar}=M_{j k}
$$

При $九 \rightarrow 0, M \sim 1 / \hbar$ целое число (9.1) приближается величиной

$$
\frac{1}{2 \pi \hbar} \int_{\Sigma_{j k}} \omega=\frac{M}{f_{j} f_{k}}
$$

где $\omega$ - классическая симплектическая форма на листе.

ДокАЗАТЕЛьство. Пусть для определенности $j=1, k=2$. Вместо комплексных координат $w_{j},(5.10)$, отвечающих резонансному базису $\rho^{(1)}, \rho^{(2)}$, (5.9), введем вещественные координаты $X_{j}, \Phi_{j}$ (см. (5.1)):

$$
w_{j}=\sqrt{X_{j}} \exp \left\{-i \Phi_{j}\right\}, \quad 0<X_{j}<\infty, \quad 0 \leqslant \Phi_{j}<2 \pi .
$$

На ребре $\left\{S_{3}=0\right\}$ классического симплекса $\boldsymbol{\Delta}[\hbar M]$, соединяющем первую и вторую вершины, имеем $w_{2}=0$ (см. (5.13)), и поэтому

$$
\left.\omega_{\hbar}\right|_{\Sigma_{12}}=\left.\hbar \frac{\partial}{\partial X_{1}}\left(X_{1} \frac{\partial}{\partial X_{1}} \ln \mathcal{K}_{R}(X)\right)\right|_{X_{2} \rightarrow 0} d X_{1} \wedge d \Phi_{1} .
$$

При движении вдоль ребра $\left\{S_{3}=0\right\}$ от первой вершины, где $S_{1}=\hbar M / f_{1}$, $S_{2}=0$, ко второй вершине, где $S_{2}=\hbar M / f_{2}, S_{1}=0$, величина $X_{1}$ меняется от $\infty$ до 0 (см. (5.13)). Поэтому

$$
\frac{1}{2 \pi \hbar} \int_{\Sigma_{12}} \omega_{\hbar}=\left.\left(X_{1} \frac{\partial}{\partial X_{1}} \ln \mathcal{K}_{R}(X)\right)\right|_{\substack{X_{1} \rightarrow \infty \\ X_{2} \rightarrow 0}} .
$$


Напомним, что полином $\mathcal{K}_{R}$ задан формулами (7.10), (7.11):

$$
\mathcal{K}_{R}(X)=\sum_{\sigma \in \mathcal{R}_{r}} c_{\sigma}^{r} X_{1}^{N_{\sigma}^{(1)}} X_{2}^{N_{\sigma}^{(2)}}, \quad c_{\sigma}^{r} \stackrel{\text { def }}{=} \frac{r !}{\hbar^{\left|\sigma_{+}\right|-\left|\sigma_{-}\right|}(r+\sigma) !},
$$

где числа $N_{\sigma}^{(j)} \in \mathbb{Z}_{+}$определяются разложением (4.3):

$$
\sigma=N_{\sigma}^{(1)} \rho^{(1)}+N_{\sigma}^{(2)} \rho^{(2)} .
$$

При $X_{2}=0$ в сумме, задающей полином $\mathcal{K}_{R}$, остаются лишь слагаемые с $N_{\sigma}^{(2)}=0$. Отсюда получаем

$$
\left.\left(X_{1} \frac{\partial}{\partial X_{1}} \ln \mathcal{K}_{R}(X)\right)\right|_{\substack{X_{1} \rightarrow \infty \\ X_{2} \rightarrow 0}}=\max _{N_{\sigma}^{(1)} \rho^{(1)} \in \mathcal{R}_{r}} N_{\sigma}^{(1)} .
$$

Включение $N_{\sigma}^{(1)} \rho^{(1)} \in \mathcal{R}_{r}$ означает, что вектор $r+N_{\sigma}^{(1)} \rho^{(1)}$ содержится в квантовом симплексе $\boldsymbol{\Delta}[M]$, где $M=\langle f, r\rangle$. Это равносильно выполнению неравенств

$$
r_{l}+N_{\sigma}^{(1)} \rho_{l}^{(1)} \geqslant 0, \quad l=1,2,3 .
$$

Напомним, что базису (5.9), который мы используем, соответствует вершина $r \equiv r^{(2)},(6.6)$. Таким образом, приходим к системе неравенств

$$
r_{1}^{(2)}+N_{\sigma}^{(1)} f_{2} \geqslant 0, \quad r_{2}^{(2)}-N_{\sigma}^{(1)} f_{1} \geqslant 0, \quad r_{3}^{(2)} \geqslant 0 .
$$

Из второго неравенства очевидно, что максимальное значение $N_{\sigma}^{(1)}$, при котором выполняется указанная система неравенств, равно $\left[\frac{r_{2}^{(2)}}{f_{1}}\right]$. Таким образом,

$$
\max _{N_{\sigma}^{(1)} \rho^{(1)} \in \mathcal{R}_{r}} N_{\sigma}^{(1)}=\left[\frac{r_{2}^{(2)}}{f_{1}}\right]
$$

что и доказывает формулу (9.1).

Если $M_{12} \geqslant 0$ в представлении (6.5), то в силу формул (6.6b), задающих вершину, имеем

$$
r_{2}^{(2)}=M_{12} f_{1}+m_{12}
$$

Здесь $0 \leqslant m_{12} \leqslant f_{1}-1$. Поэтому в данном случае $\left[\frac{r_{2}^{(2)}}{f_{1}}\right]=M_{12}$, и мы получаем формулу (9.1a).

Далее, в силу теоремы 8.2 интеграл (9.1) при $\hbar \rightarrow 0$ приближается величиной $\frac{1}{2 \pi \hbar} \int_{\Sigma_{j k}} \omega$, где $\omega-$ классическая симплектическая форма на листе. В данном случае ее удобно записать в виде (5.8) в координатах Дарбу $x_{1}, x_{2},(5.4), \varphi_{1}, \varphi_{2}$, (5.6). Выберем в (5.4) следующее семейство начальных точек: $s(\hbar M)=\hbar r^{(2)}$; тогда

$$
S_{1}=\hbar r_{1}^{(2)}+f_{2} x_{1}+\mu x_{2}, \quad S_{2}=\hbar r_{2}^{(2)}-f_{1} x_{1}+\nu x_{2}, \quad S_{3}=\hbar r_{3}^{(2)}+x_{2},
$$

откуда получаем, что на ребре $\left\{S_{3}=0\right\}$, соединяющем первую и вторую вершины, координата $x_{2}$ не меняется: $x_{2}=-\hbar r_{3}^{(2)}$. Следовательно,

$$
\left.\omega\right|_{\Sigma_{12}}=d x_{1} \wedge d \varphi_{1}
$$


Здесь $\varphi_{1}$ меняется от 0 до $2 \pi$, а при движении вдоль ребра от первой вершины ко второй координата $x_{1}$ меняется от $\hbar\left(r_{2}^{(2)}-r_{3}^{(2)} \nu\right) / f_{1}$ до $\hbar\left(\mu r_{3}^{(2)}-r_{1}^{(2)}\right) / f_{2}$. Поэтому справедлива формула (9.2):

$$
\frac{1}{2 \pi \hbar} \int_{\Sigma_{12}} \omega=\frac{1}{f_{1} f_{2}}\left(f_{1} r_{1}^{(2)}+f_{2} r_{2}^{(2)}-\left(f_{1} \mu+f_{2} \nu\right) r_{3}^{(2)}\right)=\frac{M}{f_{1} f_{2}} .
$$

(Здесь учтены диофантово уравнение (2.3) и равенство $\left\langle f, r^{(2)}\right\rangle=M$.)

Целые числа $M_{j k},(9.1 \mathrm{a})$, мы назовем главными квантовыми числами листа $\Omega_{\hbar}[M]$. Отметим, что эти числа не являются независимыми, поскольку все они однозначно задаются числом $M$ (см. также следствие 9.2).

Коэффициенты $m_{j k}$ в представлении (6.5) назовем присоединенными числами листа $\Omega_{\hbar}[M]$. Из формул (9.2) и (6.5) получаем

СледСТВИЕ 9.1. Класс когомологий классической симплектической формы на листе задается формулой

$$
\frac{1}{2 \pi \hbar} \int_{\Sigma_{j k}} \omega=M_{j k}+\frac{m_{j k}}{f_{j}}+\frac{m_{k j}}{f_{k}},
$$

где $M_{j k}$ - главные, а $m_{j k}$ - присоединенные числа листа, причем

$$
0 \leqslant \frac{m_{j k}}{f_{j}}+\frac{m_{k j}}{f_{k}} \leqslant 2-\left(\frac{1}{f_{j}}+\frac{1}{f_{k}}\right)<2 .
$$

ЗАМЕЧАНИЕ 9.1. Из (9.3) следует, что если хотя бы одна из частот $f_{j}$ не равна 1 , то класс когомологий классической симплектической формы $\omega /(2 \pi \hbar)$ на листах с ненулевыми присоединенными числами не будет целочисленным. Именно поэтому форма $\omega$ не удобна для построения квантовых геометрических конструкций.

Теперь вычислим класс квантовой 4-формы $\omega_{\hbar} \wedge \omega_{\hbar}$. Поскольку при $n=3$ листы $\Omega[\hbar M] \subset \Omega_{\hbar}[M]$ четырехмерны, то указанная 4-форма задает их квантовый объем.

ТеОРема 9.2. В трехчастотном случае $(n=3)$ квантовый обгем вычисляется следующим образом:

$$
\frac{1}{(2 \pi \hbar)^{2}} \int_{\Omega} \frac{\omega_{\hbar} \wedge \omega_{\hbar}}{2}=\frac{1}{2} \underset{\substack{(j, k, s) \\=(1,2,3)}}{\mathfrak{S}}\left(\widetilde{r}_{j}-r_{j}^{(s)}\right)\left[\frac{r_{s}^{(s)}}{f_{k}}\right],
$$

где вериины $r^{(1)}, r^{(2)}, r^{(3)}$ определены, как в замечании $6.3, \widetilde{r}-$ одна из этих трех вершин, а сумма $\mathfrak{S}$ берется по всем ииклическим перестановкам тройки индексов $(1,2,3)$. Например, если $\widetilde{r}=r^{(2)}$, то правая часть (9.4) имеет вид

$$
\frac{1}{2}\left(\left(r_{1}^{(2)}-r_{1}^{(3)}\right)\left[\frac{r_{3}^{(3)}}{f_{2}}\right]+\left(r_{2}^{(2)}-r_{2}^{(1)}\right)\left[\frac{r_{1}^{(1)}}{f_{3}}\right]\right) .
$$

Здесь и в формуле (9.4) квадратные скобки обозначают челую часть числа. Если $M_{12} \geqslant 0, M_{23} \geqslant 0$ и $M_{31} \geqslant 0$ в представлении (6.5), то (9.4) принимает вид

$$
\frac{1}{(2 \pi \hbar)^{2}} \int_{\Omega} \frac{\omega_{\hbar} \wedge \omega_{\hbar}}{2}=\frac{1}{2}\left(M_{k s}\left(M_{s j} f_{s}+m_{s j}\right)+M_{j k} m_{j s}\right) .
$$

Здесъ $(j, k, s)$ - любая циклическая перестановка тройки $(1,2,3)$. 
Доказательство теоремы 9.2 приведено в приложении.

Отметим, что при $\hbar \rightarrow 0, M \sim 1 / \hbar$ квантовый вихревой объем (9.4) приближается классическим объемом

$$
\frac{1}{(2 \pi \hbar)^{2}} \int_{\Omega} \frac{\omega \wedge \omega}{2}=\frac{M^{2}}{2 f_{1} f_{2} f_{3}} .
$$

При этом оба числа (9.4a) и (9.5) приближают размерность $d[M],(8.6)$, но не совпадают с ней.

Кроме того, теорема 9.2 дает интересные тождества для главных и присоединенных квантовых чисел.

СлЕДСтвИЕ 9.2. Пусть $n=3$. Предположим, что в представлении (6.5) все три числа $M_{12}, M_{23}, M_{31}$ неотрицательны. Тогда выполнены тождества

$$
\begin{aligned}
M_{31} M_{12} f_{1}+M_{31} m_{12}+M_{23} m_{21} & =M_{12} M_{23} f_{2}+M_{12} m_{23}+M_{31} m_{32} \\
& =M_{23} M_{31} f_{3}+M_{23} m_{31}+M_{12} m_{13} .
\end{aligned}
$$

ДокАЗАТЕЛЬСтво следует из формулы (9.4a): все три указанных числа совпадают с удвоенным квантовым объемом.

\section{$\S 10$. Квантовая резонансная алгебра}

Для любых $a \in \mathbb{R}, m \in \mathbb{Z}$ определим

$$
(a)_{m} \stackrel{\text { def }}{=}\left\{\begin{array}{lll}
(a+\hbar) \cdots(a+m \hbar) & \text { при } & m \geqslant 1, \\
1 & \text { при } & m=0, \\
a(a-\hbar) \cdots(a-\hbar(|m|-1)) & \text { при } & m \leqslant-1 .
\end{array}\right.
$$

Для векторов $s \in \mathbb{R}^{n}, \rho \in \mathbb{Z}^{n}$ положим

$$
(s)_{\rho} \stackrel{\text { def }}{=}\left(s_{1}\right)_{\rho_{1}} \cdots\left(s_{n}\right)_{\rho_{n}},
$$

где каждый из сомножителей задается с помощью (10.1).

Введенные операции (10.1), (10.1а) являются обобщением известных "символов Похгаммера" в случае отрицательных нижних индексов. При нашем модифицированном определении этих символов выполняется важное свойство, сформулированное в следующей лемме.

Лемма 10.1. Операция $\rho \rightarrow(s)_{\rho}$ обладает аналогом группового свойства:

$$
(s)_{\rho+\sigma}=\frac{(s+\hbar \sigma)_{\rho}(s)_{\sigma}}{(s+\hbar \sigma)_{[\sigma \mid \rho]}^{2}},
$$

где вектор $[\sigma \mid \rho]$ задан согласно (3.2). В частности, если $[\rho, \sigma]=0$ (см. (3.2)), $m o(s)_{\rho+\sigma}=(s+\hbar \sigma)_{\rho}(s)_{\sigma}$.

Далее, для пары векторов $\rho, \sigma \in \mathbb{Z}^{n}$ определим структурный полином $g_{\rho, \sigma}$ на $\mathbb{R}^{n}$ формулой

$$
g_{\rho, \sigma}(s) \stackrel{\text { def }}{=}(s-\hbar \rho)_{[\sigma \mid \rho]}, \quad s \in \mathbb{R}^{n} .
$$

Структурные полиномы $g_{\rho, \sigma}$ обладают целым рядом интересных свойств. Мы приведем здесь лишь несколько тождеств, которые понадобятся для дальнейшего изложения. 
Лемма 10.2. Выполнены следующие тождества:

1) если $[\rho, \sigma]=0$ (cм. (3.2)), mo $g_{\rho, \sigma}(s) \equiv 1$,

2) $g_{\rho, \sigma}(s) g_{\rho+\sigma, \varkappa}(s)=g_{\rho, \sigma+\varkappa}(s) g_{\sigma, \varkappa}(s-\hbar \rho)$,

3) $g_{\rho, \sigma}(s+\hbar \rho+\hbar \sigma)=g_{-\sigma,-\rho}(s)$,

а также их следствия:

4) $g_{\rho,-\rho}(s)=g_{-\rho, \rho}(s-\hbar \rho)$,

5) если $[\rho, \sigma]=0$, mo $g_{-\rho, \sigma}(s) g_{-\rho, \rho}(s-\hbar \sigma)=g_{\sigma,-\rho}(s) g_{-\rho, \rho}(s)$,

6) если $\left[\rho^{\prime}, \rho^{\prime \prime}\right]=\left[\sigma^{\prime}, \sigma^{\prime \prime}\right]$, mo $g_{\rho^{\prime}, \sigma^{\prime}}\left(s-\hbar \rho^{\prime \prime}\right) g_{\rho^{\prime}+\sigma^{\prime}, \sigma^{\prime \prime}}\left(s-\hbar \rho^{\prime \prime}\right) g_{\rho^{\prime \prime}, \rho^{\prime}+\sigma^{\prime}+\sigma^{\prime \prime}}(s)=$ $g_{\rho^{\prime}+\rho^{\prime \prime}, \sigma^{\prime}+\sigma^{\prime \prime}}(s)$.

Теперь зададим квантовую резонансную алгебру. Ее образующие по аналогии с классическими координатами $A_{\sigma}, S_{j}$ будем обозначать через $\mathbf{A}_{\sigma}, \mathbf{S}_{j}$, где $j=1, \ldots, n$, а вектор $\sigma$ пробегает множество $\mathcal{M}$ минимальных резонансных векторов.

Связи (3.6), (3.8), (3.9) и скобки Пуассона (3.11) мы заменим квантовыми связями и коммутационными соотношениями между образующими $\mathbf{A}_{\sigma}, \mathbf{S}$.

Квантовые связи эрмитова типа задаются следующим образом:

$$
\mathbf{S}_{j}^{*}=\mathbf{S}_{j}, \quad \mathbf{A}_{\sigma}^{*}=\mathbf{A}_{-\sigma}
$$

для любого $j=1, \ldots, n$ и любого $\sigma \in \mathcal{M}$.

Квантовые связи коммутативного типа задаются следующим образом:

$$
\prod_{\rho}\left(\mathbf{A}_{\rho}\right)^{k_{\rho}}=\prod_{\sigma}\left(\mathbf{A}_{\sigma}\right)^{m_{\sigma}}
$$

для любых семейств коммутирующих векторов $\rho, \sigma \in \mathcal{M}$ и чисел $k_{\rho}, m_{\sigma} \in \mathbb{N}$ таких, что

$$
\sum_{\rho} k_{\rho} \rho=\sum_{\sigma} m_{\sigma} \sigma
$$

Квантовые связи некоммутативного типа задаются следующим образом: если минимальные векторы $\rho$ и $\sigma$ не коммутируют и $\rho \neq-\sigma$, то выполнено соотношение

$$
\mathbf{A}_{\rho} \mathbf{A}_{\sigma}=g_{\rho, \sigma}(\mathbf{S}) \prod_{\varkappa \in \mathcal{M}_{\rho+\sigma}}\left(\mathbf{A}_{\varkappa}\right)^{n_{\varkappa}^{\rho+\sigma}}
$$

где $g_{\rho, \sigma}$ - структурный полином $(10.2)$, а $n_{\varkappa}^{\rho+\sigma}-$ коэффициенты разложения (2.2) вектора $\rho+\sigma$ по минимальным векторам из $\mathcal{M}_{\rho+\sigma}$.

Коммутационные соотношения задаются следующим образом:

$$
\left[\mathbf{S}_{j}, \mathbf{S}_{k}\right]=0, \quad\left[\mathbf{S}_{j}, \mathbf{A}_{\rho}\right]=\hbar \rho_{j} \mathbf{A}_{\rho}, \quad\left[\mathbf{A}_{-\rho}, \mathbf{A}_{\rho}\right]=\hbar F_{-\rho, \rho}(\mathbf{S})
$$

для любых $j, k=1, \ldots, n$ и $\rho \in \mathcal{M}$, где полиномы $F_{\rho, \sigma}$ заданы формулой

$$
F_{\rho, \sigma} \stackrel{\text { def }}{=} \frac{1}{\hbar}\left(g_{\rho, \sigma}-g_{\sigma, \rho}\right) .
$$

ЗАмечАниЕ 10.1. Множество связей некоммутативного типа (10.5) состоит из коммутационных соотношений

$$
\left[\mathbf{A}_{\rho}, \mathbf{A}_{\sigma}\right]=\hbar F_{\rho, \sigma}(\mathbf{S}) \prod_{\varkappa \in \mathcal{M}_{\rho+\sigma}}\left(\mathbf{A}_{\varkappa}\right)^{n_{\varkappa}^{\rho+\sigma}}
$$


и антикоммутационных соотношений

$$
\left[\mathbf{A}_{\rho}, \mathbf{A}_{\sigma}\right]_{+}=\left(g_{\rho, \sigma}(\mathbf{S})+g_{\sigma, \rho}(\mathbf{S})\right) \prod_{\varkappa \in \mathcal{M}_{\rho+\sigma}}\left(\mathbf{A}_{\varkappa}\right)^{n_{\varkappa}^{\rho+\sigma}} .
$$

Назовем соотношения (10.5а) актуальными связями некоммутативного типа.

Множество связей коммутативного типа (10.4) тоже содержит коммутационные соотношения. Это связи (10.4), соответствующие векторным равенствам $\rho+\sigma=\sigma+\rho$ для векторов $\rho, \sigma \in \mathcal{M}$ из одной нормальной подрешетки. Исключив коммутационные соотношения из множества связей (10.4), мы получим актуальные связи коммутативного типа.

Таким образом определенные актуальные связи не все являются независимыми. Число независимых актуальных связей равно $M-n+1$, где $M-$ число минимальных векторов.

ОПРЕДЕЛЕНИЕ 10.1. Резонансной алгеброй $\mathcal{A}$ назовем алгебру с инволюцией, порожденную образующими $\mathbf{A}_{\sigma}, \sigma \in \mathcal{M}, \mathbf{S}_{j}, j=1, \ldots, n$, и соотношениями (10.3)-(10.6).

По аналогии с (3.10) каждому неминимальному резонансному вектору $\sigma$ сопоставим следующий элемент $\mathbf{A}_{\sigma}$ резонансной алгебры:

$$
\mathbf{A}_{\sigma}=\left\{\begin{array}{lll}
\mathbf{I}, & \text { если } & \sigma=0 \\
\prod_{\varkappa \in \mathcal{M}_{\sigma}} \mathbf{A}_{\varkappa}^{n_{\varkappa}^{\sigma}}, & \text { если } & \sigma \neq 0
\end{array}\right.
$$

здесь подмножества $\mathcal{M}_{\sigma}$ и числа $n_{\varkappa}^{\sigma}$ определены согласно (2.2). В силу связей коммутативного типа (10.4) это обозначение корректно, т. е. не зависит от разложения вектора $\sigma$ по минимальным.

С помощью этого обозначения запишем (10.5) в виде

$$
\mathbf{A}_{\rho} \mathbf{A}_{\sigma}=g_{\rho, \sigma}(\mathbf{S}) \mathbf{A}_{\rho+\sigma}
$$

(здесь $\rho, \sigma \in \mathcal{M}, \rho \neq-\sigma)$.

Отсюда и из (10.6) получим, что для любых минимальных векторов $\rho, \sigma$ имеет место следующее коммутационное соотношение:

$$
\left[\mathbf{A}_{\rho}, \mathbf{A}_{\sigma}\right]=\hbar F_{\rho, \sigma}(\mathbf{S}) \mathbf{A}_{\rho+\sigma}
$$

где $F_{\rho, \sigma}-$ полиномы $(10.7)$.

Выпишем также ряд полезных перестановочных соотношений, вытекающих из связей $(10.4),(10.5)$ и коммутационных соотношений (10.6).

СледСтвиЕ 10.1. Образуюшие резонансной алгебры $\mathcal{A}$ удовлетворяют следуюшим перестановочным соотношениям:

(a) если $\rho, \sigma \in \mathcal{M}, \rho \neq-\sigma$, mo $g_{\sigma, \rho}(\mathbf{S}) \mathbf{A}_{\rho} \mathbf{A}_{\sigma}=g_{\rho, \sigma}(\mathbf{S}) \mathbf{A}_{\sigma} \mathbf{A}_{\rho}$;

(b) если $\rho \in \mathcal{M}, P-$ полином, $k \in \mathbb{N}, \operatorname{mo~}\left(\mathbf{A}_{\rho}\right)^{k} P(\mathbf{S})=P(\mathbf{S}-\hbar k \rho)\left(\mathbf{A}_{\rho}\right)^{k}$;

(c) если $\rho \in \mathcal{M}, k \in \mathbb{N}, m o\left[\mathbf{A}_{-\rho},\left(\mathbf{A}_{\rho}\right)^{k}\right]=g_{-\rho, \rho}(\mathbf{S})\left(\mathbf{A}_{\rho}\right)^{k-1}-\left(\mathbf{A}_{\rho}\right)^{k-1} g_{\rho,-\rho}(\mathbf{S})$.

При доказательстве соотношения (с) нужно учесть, что структурные полиномы удовлетворяют тождеству 4) из леммы 10.2.

Отметим, что при $\hbar \rightarrow 0$ для любого $s \in \mathbb{R}^{n}$ и любых $\rho, \sigma, \sigma_{j} \in \mathbb{Z}^{n}, j=1, \ldots, l$, $l \geqslant 2$, мы имеем

$$
(s)_{[\rho \mid \sigma]}=s^{\rho+\circ}+O(\hbar), \quad g_{\rho, \sigma}(s)=s^{\rho+\sigma}\left(1+\hbar \frac{\rho \& \sigma}{s}+O\left(\hbar^{2}\right)\right),
$$


где

$$
\rho \& \sigma \stackrel{\text { def }}{=} \frac{1}{2}\left(\sigma_{-}-\rho_{+}\right) \cdot(\sigma \mid \rho)+\frac{1}{2}\left(\rho_{-}-\sigma_{+}\right) \cdot(\rho \mid \sigma)+\frac{1}{2}(\rho \stackrel{\circ}{+} \sigma)-\frac{1}{2}[\rho, \sigma] .
$$

Отсюда получаем

$$
F_{\rho, \sigma}(s)=-s^{\rho+\sigma} \frac{[\rho, \sigma]}{s}+O(\hbar) .
$$

СлеДСтвИЕ 10.2. При $\hbar \rightarrow 0$ квантовая резонансная алгебра $\mathcal{A}$ соответствует пуассоновой алгебре функций на резонансном многообразии $\mathcal{N}$.

ДокАзАтельство. Достаточно сравнить квантовые и классические коммутационные соотношения (10.6), (10.9) и (3.11), а также квантовые и классические уравнения связей (10.3)-(10.5) и (3.6), (3.8), (3.9) и учесть вычисленные асимптотики. Например, при $\hbar \rightarrow 0$ коммутационное соотношение $(10.9)$ в силу асимптотической формулы

$$
F_{\rho, \sigma}(s)=-f_{\rho, \sigma}(s)+O(\hbar)
$$

переходит в третье соотношение (3.11).

Два элементарных свойства резонансной алгебры приведены в следующей теореме.

Теорема 10.1. Центр резонансной алгебры $\mathcal{A}$ содержит элемент

$$
\mathbf{C}=f_{1} \mathbf{S}_{1}+\cdots+f_{n} \mathbf{S}_{n} .
$$

Резонансная алгебра имеет представление

$$
\mathbf{A}_{\sigma} \rightarrow \widehat{A}_{\sigma}, \quad \mathbf{S}_{j} \rightarrow \widehat{S}_{j}
$$

в пространстве $L^{2}\left(\mathbb{R}^{n}\right)$ операторами симметрици резонансного осииллятора

$$
\widehat{S}_{j}=\hat{z}_{j}^{*} \hat{z}_{j}, \quad \widehat{A}_{\sigma}=\left(\hat{z}^{*}\right)^{\sigma_{+}} \hat{z}^{\sigma_{-}} .
$$

В этом представлении элемент (10.10) совпадает с гамильтонианом осциллятора (1.3).

ДокАзАТЕЛЬство. То, что операторы (10.12) удовлетворяют всем уравнениям связей и коммутационным соотношениям (10.3)-(10.6), вытекает из простых формул перестановки

$$
\hat{z} \widehat{S}_{j}=\left(\widehat{S}_{j}+\hbar\right) \hat{z}_{j}, \quad \hat{z}_{j}^{m}\left(\hat{z}_{j}^{*}\right)^{m}=\left(\widehat{S}_{j}\right)_{m}, \quad\left(\hat{z}_{j}^{*}\right)^{m} \hat{z}_{j}^{m}=\left(\widehat{S}_{j}\right)_{-m}, \quad m \in \mathbb{Z}_{+} .
$$

Теорема доказана.

\section{§ 11. Вакуумные векторы и неприводимые представления}

Рассмотрим теперь абстрактное представление резонансной алгебры $\mathcal{A}$ в некотором гильбертовом пространстве $\mathcal{H}$. Операторы представления будем обозначать теми же буквами $\mathbf{S}_{j}, \mathbf{A}_{\sigma}, j=1, \ldots, n, \sigma \in \mathcal{M}$, что и образующие алгебры $\mathcal{A}$. Для неминимального резонансного вектора $\sigma$ мы обозначаем через $\mathbf{A}_{\sigma}$ оператор, заданный формулой (10.8).

Фиксируем число $M \in \mathbb{Z}_{+}$такое, что $d[M] \neq 0$ (т. е. диофантов остов $\boldsymbol{\Delta}[M]$, $(6.2)$, не пуст). Пусть $R=(r,\{\rho\})$ - репер диофантова остова $\boldsymbol{\Delta}[M]$. Обозначим 
через $\mathcal{R}_{R}^{-}$подмножество тех векторов $\sigma$ резонансной решетки $\mathcal{R}$, для которых $r+\sigma \notin \Delta[M]$.

Предположим, что (хотя бы для одного репера $R$ ) в пространстве $\mathcal{H}$ существует нормированный вектор $\mathfrak{p}_{R}$ такой, что

$$
\begin{aligned}
\mathbf{A}_{\rho} \mathfrak{p}_{R}=0, & \rho \in \mathcal{R}_{R}^{-}, \\
\mathbf{S}_{j} \mathfrak{p}_{R}=\hbar r_{j} \mathfrak{p}_{R}, & j=1, \ldots, n,
\end{aligned}
$$

где $r$ - вершина репера $R$. Вектор $\mathfrak{p}_{R}$ назовем вакуумным.

Применяя к вакуумному вектору всевозможные операторы представления $\mathbf{A}_{\sigma}$, построим линейное подпространство $\mathcal{H}_{M} \subseteq \mathcal{H}$ :

$$
\mathcal{H}_{M}=\overline{\operatorname{span}}\left\{\mathbf{A}_{\sigma} \mathfrak{p}_{R} \mid \sigma \in \mathcal{R}\right\}
$$

Отметим, что подпространство $\mathcal{H}_{M}$ не зависит от выбора репера $R$ в условии (11.1). Это следует из леммы 12.1, которая будет доказана далее.

ПРЕДЛОЖЕНИЕ 11.1. На подпространстве $\mathcal{H}_{M}$ для любых резонансных векторов $\rho, \sigma$ справедливо равенство

$$
\mathbf{A}_{\rho} \mathbf{A}_{\sigma}=g_{\rho, \sigma}(\mathbf{S}) \mathbf{A}_{\rho+\sigma} .
$$

ЗАмечание 11.1. В случае минимальных резонансных векторов $\rho$ и $\sigma$, $\rho \neq-\sigma$, равенство (11.3) выполнено на всем пространстве $\mathcal{H}$ в силу связей некоммутативного типа (10.5).

Чтобы доказать предложение 11.1, нам понадобятся следующие три леммы.

Лемма 11.1. Пусть $\rho, \sigma-$ резонансные векторы. Тогда:

(a) если $\rho \notin \mathcal{R}_{R}^{-}$, то либо $\rho=0$, либо $-\rho \in \mathcal{R}_{R}^{-}$;

(b) если $\rho \in \mathcal{R}_{R}^{-} u[\rho, \sigma]=0$, mo $(\rho+\sigma) \in \mathcal{R}_{R}^{-}$.

Лемма 11.2. Пусть $\rho, \sigma, \theta$-резонансные векторы. Тогда:

(a) если $(\rho+\theta) \in \mathcal{R}_{R}^{-}, \theta \notin \mathcal{R}_{R}^{-}$, mo $g_{-\rho, \rho}(\hbar r+\hbar \theta)=0$;

(b) если $\sigma \notin \mathcal{R}_{R}^{-}$и $\theta \notin \mathcal{R}_{R}^{-}$, то для любого $\rho \in \mathcal{R}$ справедливо неравенство $g_{\theta-\sigma, \rho}(\hbar r+\hbar \theta)>0$.

Лемма 11.3. Пусть $\rho \in \mathcal{M}, \varkappa \in \mathcal{R}$, причем $[\rho, \varkappa]=0$. Тогда справедливо равенство

$$
\mathbf{A}_{-\rho} \mathbf{A}_{\rho} \mathbf{A}_{\varkappa} \mathfrak{p}_{R}=g_{-\rho, \rho}(\mathbf{S}) \mathbf{A}_{\varkappa} \mathfrak{p}_{R} .
$$

ДокАЗАТЕЛЬСтво. Из следствия 10.1, (b) и свойств (11.1) вакуумного вектора следует, что вместо (11.4) достаточно доказать эквивалентное равенство

$$
\mathbf{A}_{-\rho} \mathbf{A}_{\rho} \mathbf{A}_{\varkappa} \mathfrak{p}_{R}=g_{-\rho, \rho}(\hbar r+\hbar \varkappa) \mathbf{A}_{\varkappa} \mathfrak{p}_{R} .
$$

Если $(\rho+\varkappa) \in \mathcal{R}_{R}^{-}$, то левая часть (11.4а) равна нулю, поскольку из условий (11.1) имеем $\mathbf{A}_{\rho} \mathbf{A}_{\varkappa} \mathfrak{p}_{R}=\mathbf{A}_{\rho+\varkappa p_{R}}=0$. При этом и правая часть (11.4а) также равна нулю: в случае $\varkappa \in \mathcal{R}_{R}^{-}$за счет того, что $\mathbf{A}_{\varkappa \mathfrak{p}_{R}}=0$, а в случае $\varkappa \notin \mathcal{R}_{R}^{-}$ за счет зануления коэффициента, т. е. $g_{-\rho, \rho}(\hbar r+\hbar \varkappa)=0$ (здесь применена лемма $11.2,(\mathrm{a}))$. Итак, если $(\rho+\varkappa) \in \mathcal{R}_{R}^{-}$, то (11.4а) доказано.

Пусть теперь $(\rho+\varkappa) \notin \mathcal{R}_{R}^{-}$. Рассмотрим последовательно следующие возможные случаи разложения резонансного вектора $\varkappa$ в сумму коммутирующих минимальных векторов: 
$\langle 1\rangle \varkappa=k \rho$;

$\langle 2\rangle \varkappa=k \rho+\sigma$, где $[\rho, \sigma]=0, \rho \neq \sigma$;

$\langle 3\rangle \varkappa=k \rho+\sigma+\sigma^{\prime}$, где $[\rho, \sigma]=\left[\rho, \sigma^{\prime}\right]=\left[\sigma, \sigma^{\prime}\right]=0, \rho \neq \sigma, \rho \neq \sigma^{\prime}$;

Здесь $\sigma, \sigma^{\prime}, \ldots \in \mathcal{M}, k \in \mathbb{Z}_{+}$.

В случае $\langle 1\rangle$ имеем $(k+1) \rho \notin \mathcal{R}_{R}^{-}$. Значит, в силу леммы 11.1 имеем $\rho \notin$ $\mathcal{R}_{R}^{-}$, а тогда $-\rho \in \mathcal{R}_{R}^{-}$. Следовательно, во-первых, $\mathbf{A}_{-\rho} \mathfrak{p}_{R}=0$ и, во-вторых, $g_{\rho,-\rho}(\hbar r)=0$. В силу следствия $10.1,(\mathrm{~b}),($ с) отсюда вытекает справедливость равенства (11.4а) при $\varkappa=k \rho$ :

$$
\begin{aligned}
\mathbf{A}_{-\rho} \mathbf{A}_{\rho} \mathbf{A}_{k \rho} \mathfrak{p}_{R} & =\mathbf{A}_{-\rho}\left(A_{\rho}\right)^{k+1} \mathfrak{p}_{R}=\left[\mathbf{A}_{-\rho},\left(A_{\rho}\right)^{k+1}\right] \mathfrak{p}_{R} \\
& =\left(g_{-\rho, \rho}(\mathbf{S})\left(\mathbf{A}_{\rho}\right)^{k}-\left(\mathbf{A}_{\rho}\right)^{k} g_{\rho,-\rho}(\mathbf{S})\right) \mathfrak{p}_{R}=g_{-\rho, \rho}(\mathbf{S}) \mathbf{A}_{k \rho} \mathfrak{p}_{R} .
\end{aligned}
$$

В случае $\langle 2\rangle$, поскольку $\rho \neq \sigma$, применимо следствие $10.1,($ a):

$$
g_{\sigma,-\rho}(\mathbf{S}) \mathbf{A}_{-\rho} \mathbf{A}_{\sigma}=g_{-\rho, \sigma}(\mathbf{S}) \mathbf{A}_{\sigma} \mathbf{A}_{-\rho} .
$$

Используя также равенство, доказанное в случае $\langle 1\rangle$, и, далее, следствие $10.1,(\mathrm{~b})$ и тождество 5) из леммы 10.2, получим следующие равенства:

$$
\begin{aligned}
& g_{\sigma,-\rho}(\mathbf{S}) \mathbf{A}_{-\rho} \mathbf{A}_{\rho} \mathbf{A}_{k \rho+\sigma} \mathfrak{p}_{R}=g_{\sigma,-\rho}(\mathbf{S}) \mathbf{A}_{-\rho} \mathbf{A}_{\sigma} \mathbf{A}_{\rho} \mathbf{A}_{k \rho} \mathfrak{p}_{R} \\
& \quad=g_{-\rho, \sigma}(\mathbf{S}) \mathbf{A}_{\sigma} \mathbf{A}_{-\rho} \mathbf{A}_{\rho} \mathbf{A}_{k \rho} \mathfrak{p}_{R}=g_{-\rho, \sigma}(\mathbf{S}) \mathbf{A}_{\sigma} g_{-\rho, \rho}(\mathbf{S}) \mathbf{A}_{k \rho} \mathfrak{p}_{R} \\
& \quad=g_{-\rho, \sigma}(\mathbf{S}) g_{-\rho, \rho}(\mathbf{S}-\hbar \sigma) \mathbf{A}_{\sigma} \mathbf{A}_{k \rho} \mathfrak{p}_{R}=g_{\sigma,-\rho}(\mathbf{S}) g_{-\rho, \rho}(\mathbf{S}) \mathbf{A}_{k \rho+\sigma} \mathfrak{p}_{R}
\end{aligned}
$$

(здесь важно, что $[\rho, \sigma]=0)$. За счет свойства (11.1) вакуумного вектора операторный множитель $g_{\sigma,-\rho}(\mathbf{S})$ (имеющийся и в начальной, и в конечной частях равенства) можно заменить числом $g_{\sigma,-\rho}(\hbar r+\hbar k \rho+\hbar \sigma)$. В силу лемм 11.1,(b), 11.2, (b) это число отлично от нуля. Поделив (11.5) на него, придем к тождеству (11.4a) для случая $\langle 2\rangle$.

В случае $\langle 3\rangle$, действуя точно так же, как в случае $\langle 2\rangle$, и используя результат, полученный в случае $\langle 2\rangle$, придем к равенству (11.4a). Продолжая далее, по индукции получим, что (11.4a) выполнено для любых $\varkappa$, коммутирующих с $\rho$.

ДоКАЗАТЕЛЬСТво ПРЕДЛОжЕНИЯ 11.1. Прежде всего, отметим, что равенство (11.3) достаточно доказать на вакуумном векторе $\mathfrak{p}_{R}$ :

$$
\mathbf{A}_{\rho} \mathbf{A}_{\sigma} \mathfrak{p}_{R}=g_{\rho, \sigma}(\mathbf{S}) \mathbf{A}_{\rho+\sigma} \mathfrak{p}_{R}
$$

Действительно, считая (11.3) доказанным на $\mathfrak{p}_{R}$, получим, что (11.3) выполнено и на векторах вида $\mathbf{A}_{\varkappa} \mathfrak{p}_{R}$, где $\varkappa \in \mathcal{R}$ :

$$
\begin{aligned}
\mathbf{A}_{\rho} \mathbf{A}_{\sigma} \mathbf{A}_{\varkappa} \mathfrak{p}_{R} & =\mathbf{A}_{\rho} g_{\sigma, \varkappa}(\mathbf{S}) \mathbf{A}_{\sigma+\varkappa} \mathfrak{p}_{R}=g_{\sigma, \varkappa}(\mathbf{S}-\hbar \rho) \mathbf{A}_{\rho} \mathbf{A}_{\sigma+\varkappa} \mathfrak{p}_{R} \\
& =g_{\sigma, \varkappa}(\mathbf{S}-\hbar \rho) g_{\rho, \sigma+\varkappa}(\mathbf{S}) \mathbf{A}_{\rho+\sigma+\varkappa} \mathfrak{p}_{R} \\
& =g_{\rho, \sigma}(\mathbf{S}) g_{\rho+\sigma, \varkappa}(\mathbf{S}) \mathbf{A}_{\rho+\sigma+\varkappa} \mathfrak{p}_{R}=g_{\rho, \sigma}(\mathbf{S}) \mathbf{A}_{\rho+\sigma} \mathbf{A}_{\varkappa} \mathfrak{p}_{R} .
\end{aligned}
$$

Здесь использованы следствие $10.1,(\mathrm{~b})$ и лемма 10.2,2).

Пусть $\rho, \sigma \in \mathbb{Z}^{n}$. Число таких $j$, для которых $[\rho, \sigma]_{j} \neq 0$, назовем индексом некоммутативности $m(\rho, \sigma)$ векторов $\rho, \sigma ; m(\rho, \sigma) \in\{0,1, \ldots, n\}$.

Нетрудно убедиться в справедливости следующих утверждений.

Лемма 11.4. Пусть $\rho=\rho^{\prime}+\rho^{\prime \prime}, \sigma=\sigma^{\prime}+\sigma^{\prime \prime}$, причем $\left[\rho^{\prime}, \rho^{\prime \prime}\right]=\left[\sigma^{\prime}, \sigma^{\prime \prime}\right]=0$. Тогда: 
(a) $m\left(\rho^{\prime}+\sigma^{\prime}, \sigma^{\prime \prime}\right) \leqslant m(\rho, \sigma)$;

(b) $m\left(\rho^{\prime \prime}, \rho^{\prime}+\sigma\right) \leqslant m(\rho, \sigma)$;

(c) если существует $j$ такое, что $[\rho, \sigma]_{j} \neq 0, \rho_{j}^{\prime}+\sigma_{j}^{\prime}=0$, mо $m\left(\rho^{\prime}+\sigma^{\prime}, \sigma^{\prime \prime}\right) \leqslant$ $m(\rho, \sigma)$

(d) если существует $j$ такое, что $[\rho, \sigma]_{j} \neq 0, \rho_{j}^{\prime \prime}=0$, mo $m\left(\rho^{\prime \prime}, \rho^{\prime}+\sigma\right) \leqslant$ $m(\rho, \sigma)$.

Доказательство равенства (11.3а) проведем путем индукции по величине индекса некоммутативности $m(\rho, \sigma)$.

Если $m(\rho, \sigma)=0$, т. е. $[\rho, \sigma]=0$, то (11.3) вытекает из связей (10.4) коммутативного типа и тождества 1$)$ из леммы 10.2 .

Предположение индукции формулируется следующим образом:

(П1) пусть формула (11.3а) выполнена для всех $\rho, \sigma \in \mathcal{R}$ таких, что $m(\rho, \sigma) \leqslant m$.

Исходя из этого, докажем (11.3а) для таких $\rho, \sigma \in \mathcal{R}$, что $m(\rho, \sigma)=m+1$.

Поскольку $m(\rho, \sigma) \geqslant 1$, то хотя бы одна из компонент коммутатора $[\rho, \sigma]$ отлична от нуля, т. е.

$$
\exists j: \quad[\rho, \sigma]_{j} \neq 0 .
$$

Поэтому векторы $\rho$ и $\sigma$ можно представить в виде

$$
\begin{aligned}
& \rho=\rho^{\prime}+\rho^{\prime \prime}, \quad \rho^{\prime} \in \mathcal{M}, \quad \rho^{\prime \prime} \in \mathcal{R}, \quad\left[\rho^{\prime}, \rho^{\prime \prime}\right]=0, \quad \rho_{j}^{\prime} \neq 0, \\
& \sigma=\sigma^{\prime}+\sigma^{\prime \prime}, \quad \sigma^{\prime} \in \mathcal{M}, \quad \sigma^{\prime \prime} \in \mathcal{R}, \quad\left[\sigma^{\prime}, \sigma^{\prime \prime}\right]=0, \quad \sigma_{j}^{\prime} \neq 0 .
\end{aligned}
$$

Следовательно,

$$
\mathbf{A}_{\rho} \mathbf{A}_{\sigma} \mathfrak{p}_{R}=\mathbf{A}_{\rho^{\prime \prime}} \mathbf{A}_{\rho^{\prime}} \mathbf{A}_{\sigma^{\prime}} \mathbf{A}_{\sigma^{\prime \prime}} \mathfrak{p}_{R}
$$

В правой части этого равенства произведение $\mathbf{A}_{\rho^{\prime}} \mathbf{A}_{\sigma^{\prime}}$ можно заменить на $g_{\rho^{\prime}, \sigma^{\prime}}(\mathbf{S}) \mathbf{A}_{\rho^{\prime}+\sigma^{\prime}}:$ если $\rho^{\prime} \neq-\sigma^{\prime}$, то в силу связей (10.6) некоммутативного типа, а если $\rho^{\prime}=-\sigma^{\prime}$, то в силу леммы 11.3 (см. также обозначение (10.8)). В результате (используя следствие $10.1,(\mathrm{~b})$ ) получим следующее равенство:

$$
\mathbf{A}_{\rho} \mathbf{A}_{\sigma} \mathfrak{p}_{R}=g_{\rho^{\prime}, \sigma^{\prime}}\left(\mathbf{S}-\hbar \rho^{\prime \prime}\right) \mathbf{A}_{\rho^{\prime \prime}} \mathbf{A}_{\rho^{\prime}+\sigma^{\prime}} \mathbf{A}_{\sigma^{\prime \prime}} \mathfrak{p}_{R}
$$

Дополнительно проведем индукцию по величине $M_{j}(\rho, \sigma) \stackrel{\text { def }}{=}\left|[\rho, \sigma]_{j}\right|$.

Если $M_{j}(\rho, \sigma)=1$, то, очевидно, $\rho_{j}^{\prime}=-\sigma_{j}^{\prime}, \rho_{j}^{\prime \prime}=0$. Отсюда в силу утверждений (c), (d) леммы 11.4 следует, что выполнены неравенства

$$
m\left(\rho^{\prime}+\sigma^{\prime}, \sigma^{\prime \prime}\right)<m+1, \quad m\left(\rho^{\prime \prime}, \rho^{\prime}+\sigma\right)<m+1,
$$

и, значит, по предположению индукции (П1) имеем

$$
\begin{aligned}
\mathbf{A}_{\rho^{\prime}+\sigma^{\prime}} \mathbf{A}_{\sigma^{\prime \prime}} \mathfrak{p}_{R} & =g_{\rho^{\prime}+\sigma^{\prime}, \sigma^{\prime \prime}}(\mathbf{S}) \mathbf{A}_{\rho^{\prime}+\sigma^{\prime}+\sigma^{\prime \prime}} \mathfrak{p}_{R}, \\
\mathbf{A}_{\rho^{\prime \prime}} \mathbf{A}_{\rho^{\prime}+\sigma} \mathfrak{p}_{R} & =g_{\rho^{\prime \prime}, \rho^{\prime}+\sigma}(\mathbf{S}) \mathbf{A}_{\rho^{\prime \prime}+\rho^{\prime}+\sigma} \mathfrak{p}_{R} .
\end{aligned}
$$

Подставляя эти равенства в (11.7) и используя следствие 10.1,(b), а также тождество 6) из леммы 10.2, получим искомое равенство (11.3а) для случая $m(\rho, \sigma)=m+1, M_{j}(\rho, \sigma)=1$ :

$$
\begin{aligned}
\mathbf{A}_{\rho} \mathbf{A}_{\sigma} \mathfrak{p}_{R} & =g_{\rho^{\prime}, \sigma^{\prime}}\left(\mathbf{S}-\hbar \rho^{\prime \prime}\right) \mathbf{A}_{\rho^{\prime \prime}} g_{\rho^{\prime}+\sigma^{\prime}, \sigma^{\prime \prime}}(\mathbf{S}) \mathbf{A}_{\rho^{\prime}+\sigma} \mathfrak{p}_{R} \\
& =g_{\rho^{\prime}, \sigma^{\prime}}\left(\mathbf{S}-\hbar \rho^{\prime \prime}\right) g_{\rho^{\prime}+\sigma^{\prime}, \sigma^{\prime \prime}}\left(\mathbf{S}-\hbar \rho^{\prime \prime}\right) g_{\rho^{\prime \prime}, \rho^{\prime}+\sigma}(\mathbf{S}) \mathbf{A}_{\rho+\sigma} \mathfrak{p}_{R} \\
& =g_{\rho^{\prime}+\rho^{\prime \prime}, \sigma^{\prime}+\sigma^{\prime \prime}}(\mathbf{S}) \mathbf{A}_{\rho+\sigma} \mathfrak{p}_{R}=g_{\rho, \sigma}(\mathbf{S}) \mathbf{A}_{\rho+\sigma} \mathfrak{p}_{R} .
\end{aligned}
$$


Предположение индукции формулируется следующим образом:

(П2) пусть формула (11.3а) выполнена для всех $\rho, \sigma \in \mathcal{R}$ таких, что

$$
m(\rho, \sigma)=m+1 \text { и } M_{j}(\rho, \sigma) \leqslant M_{j} .
$$

Рассмотрим такие $\rho, \sigma \in \mathcal{R}$, что $m(\rho, \sigma)=m+1$ и $M_{j}(\rho, \sigma)=M_{j}+1$. Тогда, во-первых, из утверждений $(\mathrm{a}),(\mathrm{b})$ леммы 11.4 получим, что выполнены неравенства

$$
m\left(\rho^{\prime}+\sigma^{\prime}, \sigma^{\prime \prime}\right) \leqslant m+1, \quad m\left(\rho^{\prime \prime}, \rho^{\prime}+\sigma\right) \leqslant m+1,
$$

и, во-вторых, из (11.6) получим неравенства

$$
M_{j}\left(\rho^{\prime}+\sigma^{\prime}, \sigma^{\prime \prime}\right) \leqslant M_{j}, \quad M_{j}\left(\rho^{\prime \prime}, \rho^{\prime}+\sigma\right) \leqslant M_{j} .
$$

Значит, выполнено одно из двух предположений индукции: либо (П1), либо (П2). В любом из этих двух случаев справедливы равенства (11.8). Подставляя их в $(11.7)$, приходим к формуле (11.3а) для случая $m(\rho, \sigma)=m+1, M_{j}(\rho, \sigma)=$ $M_{j}+1$.

Итак, в силу принципа математической индукции формула (11.3а) выполнена при всех значениях $m(\rho, \sigma) \in\{0,1, \ldots, n\}, n \in \mathbb{N}$.

СлЕДСТВИЕ 11.1. Подпространство $\mathcal{H}_{M},(11.2)$, инвариантно и минимально относительно представления резонансной алгебры (т.е. это подпространство неприводимого представления алгебры $\mathcal{A})$. На векторы из $\mathcal{H}_{M}$ операторы представления действуют следующим образом:

$$
\begin{gathered}
\mathbf{S}_{j} \mathbf{A}_{\sigma} \mathfrak{p}_{R}=\hbar\left(r_{j}+\sigma_{j}\right) \mathbf{A}_{\sigma} \mathfrak{p}_{R}, \\
\mathbf{A}_{\rho} \mathbf{A}_{\sigma} \mathfrak{p}_{R}=g_{\rho, \sigma}(\hbar r+\hbar \rho+\hbar \sigma) \mathbf{A}_{\rho+\sigma} \mathfrak{p}_{R} .
\end{gathered}
$$

Отсюда и из (11.1), а также из леммы 10.2,4) вытекает

СлеДСтвиЕ 11.2. Пусть $\sigma, \theta \in \mathcal{R}$. Тогда:

(a) если $(r+\sigma) \notin \boldsymbol{\Delta}[M]$, mo $\mathbf{A}_{\sigma} \mathfrak{p}_{R}=0$;

(b) если $(r+\sigma) \in \boldsymbol{\Delta}[M]$, mo $\left\|\mathbf{A}_{\sigma} \mathfrak{p}_{R}\right\|=(\hbar r)_{-\sigma}^{-1 / 2}$;

(c) если $\sigma \neq \theta$, mо $\left(\mathbf{A}_{\sigma} \mathfrak{p}_{R}, \mathbf{A}_{\theta} \mathfrak{p}_{R}\right)=0$.

\section{§ 12. Когерентные состояния}

Рассмотрим неприводимое представление резонансной алгебры в пространстве $\mathcal{H}_{M} \subset \mathcal{H},(11.2)$, порожденном вакуумным вектором. Каждой точке остова $t \in \boldsymbol{\Delta}[M]$ сопоставим вектор из подпространства $\mathcal{H}_{M}$ по формуле

$$
\mathfrak{p}^{t} \stackrel{\text { def }}{=}(\hbar r)_{t-r}^{-1 / 2} \mathbf{A}_{t-r} \mathfrak{p}_{R},
$$

где $\mathfrak{p}_{R}$ - вакуумный вектор, а $r$ - вершина репера $R$.

ПРЕДЛОЖЕНИЕ 12.1. 1) Векторы $\mathfrak{p}^{t},(12.1)$, образуют в $\mathcal{H}_{M},(11.2)$, ортонормированный базис.

2) Операторы представления алгебры $\mathcal{A}$ в базисе $\left\{\mathfrak{p}^{t} \mid t \in \boldsymbol{\Delta}[M]\right\}$ имеют вид

$$
\begin{aligned}
& \mathbf{S}_{j} \mathfrak{p}^{t}=\hbar t_{j} \mathfrak{p}^{t}, \quad j=1, \ldots, n, \\
& \mathbf{A}_{\rho} \mathfrak{p}^{t}=(\hbar t)_{\rho}^{1 / 2} \mathfrak{p}^{\rho+t}, \quad \rho \in \mathcal{R} .
\end{aligned}
$$


ДокАЗАТЕЛЬСтво. В пояснении нуждается только формула (12.3). Пусть $(\rho+t) \in \boldsymbol{\Delta}[M]$. Тогда в силу следствия 11.1 и определения (12.1) имеем

$$
\mathbf{A}_{\rho} \mathfrak{p}^{t}=\sqrt{\frac{(\hbar r)_{\rho+t-r}}{(\hbar r)_{t-r}}} g_{\rho, t-r}(\hbar \rho+\hbar t) \mathfrak{p}^{\rho+t} .
$$

Согласно лемме 10.1 и определению (10.2) выполнено тождество

$$
\left(g_{\rho, \sigma}(s)\right)^{2}=\frac{(s-\hbar \rho)_{\rho}(s-\hbar \rho-\hbar \sigma)_{\sigma}}{(s-\hbar \rho-\hbar \sigma)_{\rho+\sigma}}, \quad s \in \mathbb{R}^{n}, \quad \rho, \sigma \in \mathbb{Z}^{n} .
$$

Отсюда и из леммы 11.2 получаем

$$
g_{\rho, t-r}(\hbar \rho+\hbar t)=\sqrt{\frac{(\hbar t)_{\rho}(\hbar r)_{t-r}}{(\hbar r)_{\rho+t-r}}}
$$

и приходим к формуле (12.3).

Пусть теперь $(\rho+t) \notin \boldsymbol{\Delta}[M]$. Тогда левая часть равенства (12.3) равна нулю согласно следствию 11.2, а правая часть (12.3) равна нулю за счет коэффициента: $(\hbar t)_{\rho}=0$. Таким образом, формула (12.3) выполнена и в этом случае.

ЛЕмма 12.1. Пусть выполнено условие существования вакуумного вектоpa $\mathfrak{p}_{R},(11.1)$, для некоторого репера $R$ резонансного остова $\boldsymbol{\Delta}[M]$. Тогда для любого другого репера $L$ остова $\boldsymbol{\Delta}[M]$ существует вакуумный вектор $\mathfrak{p}_{L}, a$ именно

$$
\mathfrak{p}_{L}=\mathfrak{p}^{l} .
$$

Здесъ l-вершина резонансного репера L, a вектор $\mathfrak{p}^{l}$ задается бормулой (12.1).

ДоказАтельство. Напомним, что $\sigma \in \mathcal{R}_{L}^{-} \Longleftrightarrow(l+\sigma) \notin \boldsymbol{\Delta}[M]$. Отсюда в силу предложения 12.1 получаем, что вектор $\mathfrak{p}_{L}=\mathfrak{p}^{l}$ удовлетворяет условиям (11.1) для вакуумного вектора:

$$
\begin{gathered}
\mathbf{A}_{\sigma} \mathfrak{p}_{L}=0, \quad 0 \in \mathcal{R}_{L}^{-}, \\
\mathbf{S}_{j} \mathfrak{p}_{L}=\hbar l \mathfrak{p}_{L}, \quad j=1, \ldots, n .
\end{gathered}
$$

Кроме того, согласно предложению $12.1,1)$ вектор $\mathfrak{p}_{L}$ нормирован: $\left\|\mathfrak{p}_{L}\right\|=1$. Значит, $\mathfrak{p}_{L}$ - вакуумный вектор, соответствующий реперу $L$.

Лемма 12.2. Вектор $\mathfrak{p}^{t},(12.1)$, не зависит от выбора репера $R$.

ДокАЗАТЕЛЬСтво. В силу формулы (12.3) имеем

$$
\mathbf{A}_{t-l} \mathfrak{p}^{l}=(\hbar l)_{t-l}^{1 / 2} \mathfrak{p}^{t}
$$

Следовательно, вектор $\mathfrak{p}^{t}$ может быть задан формулой

$$
\mathfrak{p}^{t}=(\hbar l)_{t-l}^{-1 / 2} \mathbf{A}_{t-l} \mathfrak{p}_{L}
$$

через вакуумный вектор $\mathfrak{p}_{L}=\mathfrak{p}^{l}$, отвечающий произвольному реперу $L$. Сравнивая полученное выражение с $(12.1)$, приходим к выводу о независимости $\mathfrak{p}^{t}$ от выбора репера. 
ПримеР 12.1. В случае, если $\mathcal{H}=L^{2}\left(\mathbb{R}^{n}\right)$ и представление алгебры $\mathcal{A}$ задано формулами (10.12), базисные векторы $\mathfrak{p}^{t}$ являются функциями на $\mathbb{R}^{n}$ :

$$
\mathfrak{p}^{t}(q)=\frac{1}{\sqrt{2^{|t|} t !}} \prod_{j=1}^{n} \sqrt[4]{\frac{f_{j}}{\pi \hbar}} H_{t_{j}}\left(\sqrt{\frac{f_{j}}{\hbar}} q_{j}\right) \exp \left\{-\frac{1}{2 \hbar} \sum_{j=1}^{n} f_{j} q_{j}^{2}\right\}, \quad q \in \mathbb{R}^{n} .
$$

Здесь $H_{k}$ - стандартные полиномы Эрмита, определяемые следующим образом:

$$
H_{k}(\xi) \stackrel{\text { def }}{=}(-1)^{k} e^{\xi^{2}} \frac{d^{k}}{d \xi^{k}}\left(e^{-\xi^{2}}\right) .
$$

Действительно, в этом случае вакуумный вектор задан формулой

$$
\mathfrak{p}_{R}=c(r)\left(\hat{z}^{*}\right)^{r} \chi_{0},
$$

где

$$
c(r) \stackrel{\text { def }}{=} \sqrt[4]{\frac{f_{1} \cdots f_{n}}{(\pi \hbar)^{n}}} \frac{1}{\sqrt{\hbar^{|r|} r !}}, \quad \chi_{0}(q)=\exp \left\{-\frac{1}{2 \hbar} \sum_{j=1}^{n} f_{j} q_{j}^{2}\right\} .
$$

Из определения (12.1) следует

$$
\begin{aligned}
\mathfrak{p}^{t} & =c(r)(\hbar r)_{t-r}^{-1 / 2}\left(\hat{z}^{*}\right)^{(t-r)_{+}} \hat{z}^{(t-r)-}\left(\hat{z}^{*}\right)^{r} \chi_{0} \\
& =c(t)\left(\hat{z}^{*}\right)^{t} \chi_{0}=c(t) \frac{\hbar^{|t| / 2}}{2^{|t| / 2}} \prod_{j=1}^{n} H_{t_{j}}\left(\sqrt{\frac{f_{j}}{\hbar}} q_{j}\right) \chi_{0},
\end{aligned}
$$

что дает указанную выше формулу для $\mathfrak{p}^{t}(q)$.

Напомним, что множеству всех вершин $t \in \boldsymbol{\Delta}[M]$ мы сопоставили в $\S 7$ ортонормированный базис $\left\{U^{t}\right\}$ в пространстве $\mathcal{L}\left(\Omega_{\hbar}\right)$ голоморфных сечений пучка $\Pi\left(\Omega_{\hbar}\right)$.

Обозначим через $\Pi\left(\Omega_{\hbar}, \mathcal{H}\right)=\Pi\left(\Omega_{\hbar}\right) \otimes \mathcal{H}$ пучок ростков голоморфных функций на $\Omega_{\hbar}$ со значениями в $\mathcal{H}$ с теми же, что и раньше, скалярными функциями склейки (7.2). Пространство его сечений $\Psi=\left\{\Psi_{R}\right\}$, снабженное естественной гильбертовой нормой

$$
\|\Psi\|=\left(\frac{1}{(2 \pi \hbar)^{n-1}} \int_{\Omega_{\hbar}} \frac{\left\|\Psi_{R}\right\|_{\mathcal{H}}^{2}}{\mathcal{K}_{R}} d m_{\hbar}\right)^{1 / 2},
$$

будем обозначать через $\mathcal{L}\left(\Omega_{\hbar}, \mathcal{H}\right)$.

Очевидно, для подпространства $\mathcal{H}_{M} \subset \mathcal{H}$ мы имеем вложение $\mathcal{L}\left(\Omega_{\hbar}, \mathcal{H}_{M}\right) \subset$ $\mathcal{L}\left(\Omega_{\hbar}, \mathcal{H}\right)$.

ОПРЕДЕЛЕНИЕ 12.1. Когерентными состояниями алгебры $\mathcal{A}$, отвечающими квантовому листу $\Omega_{\hbar}=\Omega_{\hbar}[M]$, назовем семейство векторов

$$
\mathfrak{P} \stackrel{\text { def }}{=} \sum_{t \in \Delta[M]} U^{t} \mathfrak{p}^{t}
$$

Формула (12.4) задает элемент пространства $\mathcal{L}\left(\Omega_{\hbar}, \mathcal{H}_{M}\right)$. В локальной карте с номером $R=(r,\{\rho\})$ векторы $\mathfrak{P}=\mathfrak{P}_{R}(w)$ являются голоморфными функциями от комплексных координат $w$ согласно (7.1):

$$
\mathfrak{P}_{R}(w)=\sum_{t \in \Delta[M]} U_{R}^{t}(w) \mathfrak{p}^{t}=\sum_{t \in \Delta[M]} \sqrt{\frac{\hbar^{|r|} r !}{\hbar^{|t|} t !}} \prod_{k=1}^{n-1} w^{N_{t-r}^{(k)}} \mathfrak{p}^{t} .
$$


Здесь неотрицательные показатели степени $N_{t-r}^{(k)}$ определяются разложением (4.3) резонансного вектора $t-r$ по базису $\{\rho\}$ в вершине $r$.

ПримеР 12.2. Пусть $n=3, f_{1}=1$. Тогда для каждого $M \in \mathbb{Z}_{+}$диофантов остов $\boldsymbol{\Delta}[M]$ состоит из точек

$$
\left(M-f_{2} j-f_{3} k, j, k\right), \quad \text { где } \quad j \in \mathbb{Z}_{+}, \quad k \in \mathbb{Z}_{+}, \quad f_{2} j+f_{3} k \leqslant M .
$$

В качестве резонансного репера $R=(r,\{\rho\})$ выберем вершину $r=(M, 0,0)$ и базис $(4.8): \rho^{(1)}=\left(-f_{2}, 1,0\right), \rho^{(2)}=\left(-f_{3}, 0,1\right)$. Тогда в локальной карте с номером $R$ для когерентных состояний (12.5) получим следующую формулу:

$$
\mathfrak{P}_{R}(w)=\sum_{\substack{j \in \mathbb{Z}_{+}, k \in \mathbb{Z}_{+} \\ f_{2} j+f_{3} k \leqslant M}} \frac{1}{j ! k !}\left(\frac{w_{1} \mathbf{A}_{\rho^{(1)}}}{\hbar}\right)^{j}\left(\frac{w_{2} \mathbf{A}_{\rho^{(2)}}}{\hbar}\right)^{k} \mathfrak{p}^{r} .
$$

Здесь $\mathfrak{p}^{r}$ - вакуумный вектор, подчиненный уравнениям (11.1):

$$
\mathbf{S}_{1} \mathfrak{p}^{r}=\hbar M \mathfrak{p}^{r}, \quad \mathbf{S}_{2} \mathfrak{p}^{r}=\mathbf{S}_{3} \mathfrak{p}^{r}=0,
$$

и условию нормировки $\left\|\mathfrak{p}^{r}\right\|=1$, а $w_{1}, w_{2}$ - комплексные координаты, связанные с резонансным базисом $\rho^{(1)}, \rho^{(2)}$ по формулам (4.4). Отметим, что векторы $\rho^{(1)}$ и $\rho^{(2)}$ коммутируют между собой (в смысле $\left.(3.2)\right):\left[\rho^{(1)}, \rho^{(2)}\right]=0$, и потому операторы $\mathbf{A}_{\rho^{(1)}}$ и $\mathbf{A}_{\rho^{(2)}}$ также коммутируют, т. е.

$$
\left[\mathbf{A}_{\rho^{(1)}}, \mathbf{A}_{\rho^{(2)}}\right]=0 .
$$

Кроме того, отметим, что при всех $j, k \in \mathbb{Z}_{+}, f_{2} j+f_{3} k>M$, имеет место равенство $\left(\mathbf{A}_{\rho^{(1)}}\right)^{j}\left(\mathbf{A}_{\rho^{(2)}}\right)^{k} \mathfrak{p}^{r}=0$. Поэтому суммирование в формуле (12.6) по двум индексам $j$ и $k$ можно продолжить до $+\infty$. В результате для когерентных состояний получим следующее выражение:

$$
\mathfrak{P}_{R}(w)=\exp \left\{\frac{1}{\hbar}\left(w_{1} \mathbf{A}_{\rho^{(1)}}+w_{2} \mathbf{A}_{\rho^{(2)}}\right)\right\} \mathfrak{p}^{r} .
$$

Упомянем общие свойства когерентных состояний [39], [40].

Лемма 12.3. (а) Скалярное произведение $\mathcal{K}=(\mathfrak{P}, \mathfrak{P})_{\mathcal{H}}$ когерентных состояний совпадает с воспроизводящим ядром (7.7), (7.9) пространства $\mathcal{L}\left(\Omega_{\hbar}\right)$.

(b) Проекторы $\boldsymbol{\pi}_{\hbar}$ на одномерные подпространства в $\mathcal{H}_{M}$, порожденные когерентными состояниями, в сумме задают проектор $\boldsymbol{\Pi}_{\hbar}[M]$ на все $\mathcal{H}_{M}$ (т.е. на всю неприводимую компоненту представления алгебры $\mathcal{A}$ в гилъбертовом пространстве $\mathcal{H})$ :

$$
\frac{1}{(2 \pi \hbar)^{n-1}} \int_{\Omega_{\hbar}[M]} \boldsymbol{\pi}_{\hbar} d m_{\hbar}=\boldsymbol{\Pi}_{\hbar}[M]
$$

\section{§ 13. Реализация неприводимых представлений над квантовыми листами}

Покажем теперь, как универсально реализовать неприводимые представления квантовой резонансной алгебры $\mathcal{A}$ в пространствах антиголоморфных сечений над квантовыми листами $\Omega_{\hbar}[M]$. 
Фиксируем в $\Omega_{\hbar}[M]$ локальную карту с номером $R=(r,\{\rho\})$. Напомним, что здесь $r$ - вершина диофантова остова $\boldsymbol{\Delta}$, а $\{\rho\}-$ резонансный базис. Через $w$ обозначим комплексные координаты в данной карте.

Определим вектор-функции:

$$
s(m) \stackrel{\text { def }}{=} \hbar r+\hbar \sum_{k=1}^{n-1} m_{k} \rho^{(k)}, \quad m \in \mathbb{Z}^{n} .
$$

Теорема 13.1. Дифберенииальные операторы

$$
\begin{gathered}
\stackrel{\circ}{S}_{j} \stackrel{\text { def }}{=} s_{j}\left(\bar{w} \frac{\partial}{\partial \bar{w}}\right), \quad j=1, \ldots, n, \\
\stackrel{\circ}{A_{\sigma}} \stackrel{\text { def }}{=}(\stackrel{\circ}{S})_{\sigma_{-}} \overline{W_{\sigma}}, \quad \sigma \in \mathcal{M},
\end{gathered}
$$

заданные в локальных картах на $\Omega_{\hbar}=\Omega_{\hbar}[M]$, согласованы на пересечениях карт и задают неприводимое представление квантовой резонансной алгебры $\mathcal{A}$ в гилъбертовом пространстве $\mathcal{L}^{*}\left(\Omega_{\hbar}\right)$ (антиголоморбных) сечений пучка $\Pi^{*}\left(\Omega_{\hbar}\right)$. Для этого представления вакуумным вектором, отвечающим реперу $R$, служит сечение пучка $\Pi^{*}\left(\Omega_{\hbar}\right)$, которое в карте в номером $R$ задано единичной функиией.

Во второй формуле из (13.1) используются обозначения (10.1а) и (4.5).

ДокАЗАтЕЛЬСтво теоремы 13.1. Проверка согласованности на пересечениях карт вполне рутинна. Выполнение уравнений связи и коммутационных соотношений (10.4)-(10.6) следует из перестановочных формул

$$
\bar{w}_{k} \frac{\partial}{\partial \bar{w}_{k}} \circ \overline{W_{\sigma}}=\bar{W}_{\sigma} \circ\left(\bar{w}_{k} \frac{\partial}{\partial \bar{w}_{k}}+N_{\sigma}^{(k)}\right), \quad \stackrel{\circ}{S}_{j} \circ \overline{W_{\sigma}}=\overline{W_{\sigma}} \circ\left(\stackrel{\circ}{S}_{j}+\hbar \sigma_{j}\right),
$$

где числа $N_{\sigma}^{(k)}$ определены согласно (4.3). Условия эрмитовости (10.3) для операторов (13.1) достаточно проверить на векторах $\overline{U^{t}}$ ортонормированного базиса $(7.1),(7.5)$. Для операторов $\stackrel{\circ}{S}_{j}$ эти векторы собственные:

$$
\stackrel{\circ}{S}_{j} \overline{U^{t}}=\hbar t_{j} \overline{U^{t}}
$$

Операторы $\stackrel{\circ}{A}$ действуют следующим образом:

$$
\stackrel{\circ}{A}_{\sigma} \overline{U^{t}}=\sqrt{(\hbar t)_{\sigma}} \overline{U^{t+\sigma}} .
$$

Отсюда в силу свойства $(s-\hbar \sigma)_{\sigma}=(s)_{-\sigma}$ символов $(10.1 \mathrm{a})$ получаем

$$
\begin{aligned}
\left(\stackrel{\circ}{A}_{\sigma} \overline{U^{k}}, \overline{U^{t}}\right) & =\sqrt{(\hbar k)_{\sigma}}\left(\overline{U^{k+\sigma}}, \overline{U^{t}}\right)=\sqrt{(\hbar k)_{\sigma}} \delta_{k+\sigma, t} \\
& =\sqrt{(\hbar t)_{-\sigma}} \delta_{k, t-\sigma}=\left(\overline{U^{k}}, \stackrel{\circ}{A_{-\sigma} \overline{U^{t}}}\right),
\end{aligned}
$$

т. е. $\stackrel{\circ}{A}_{\sigma}^{*}=\stackrel{\circ}{A}-\sigma$.

При $t=r$, поскольку $(\hbar r)_{\sigma}=0$ для любого $\sigma \in \mathcal{R}_{R}^{-}$, из $(13.2),(13.3)$ получаем

$$
\stackrel{\circ}{S}_{j} \overline{U^{r}}=\hbar r_{j} \overline{U^{r}}, \quad \stackrel{\circ}{A}_{\sigma} \overline{U^{r}}=0, \quad j=1, \ldots, n, \quad \sigma \in \mathcal{R}_{R}^{-},
$$

т. е. сечение $\overline{U^{r}}$ является вакуумным вектором для операторов (13.1). Это сечение в карте с номером $R$ тождественно равно единице: $\overline{U_{R}^{r}}=1$ (см. (7.1)). 
ЗАмЕчАниЕ 13.1. Поскольку скалярное произведение в пространстве $\mathcal{L}^{*}\left(\Omega_{\hbar}\right)$ представимо также в виде интеграла (8.1), то условие эрмитовости представления (13.1) можно записать в виде некоторых уравнений для плотности $\mathcal{J}$, а также и для воспроизводящего ядра $\mathcal{K}$ (это известная процедура; см. [25]). Система уравнений на $\mathcal{J}$ и $\mathcal{K}$ будет следующей:

$$
\begin{aligned}
\left(\stackrel{\circ}{S}_{i}\right)^{T} \mathcal{J} & =\left(\bar{\circ}_{S_{i}}\right)^{T} \mathcal{J}, & \left(\stackrel{\circ}{A}_{\sigma}\right)^{T} \mathcal{J} & =\left(\overline{\stackrel{\circ}{A}_{-\sigma}}\right)^{T} \mathcal{J}, \\
\stackrel{\circ}{S}_{i} \mathcal{K} & =\stackrel{\circ}{S}_{i} \mathcal{K}, & \stackrel{\circ}{A}_{\sigma} \mathcal{K} & ={\stackrel{\circ}{A_{-\sigma}} \mathcal{K} .}
\end{aligned}
$$

Здесь через $(\ldots)^{T}$ обозначена операция транспонирования дифференциального оператора относительно стандартной меры $d w$ (или $d \bar{w}$ ) в локальных комплексных координатах, а чертой обозначено комплексное сопряжение оператора.

В выписанной системе левая колонка уравнений (в которой $i=1, \ldots, n$ ) обеспечивает тот факт, что плотность $\mathcal{J}$ и ядро $\mathcal{K}$ зависят лишь от модулей комплексных координат $w$, т. е. от вещественных переменных $X_{j}=\left|w_{j}\right|^{2}$. Правую колонку уравнений (в которой $\sigma \in \mathcal{M}$ ) достаточно записать только для векторов базиса $\rho^{(j)}$; она будет иметь следующий вид:

$$
\begin{gathered}
{\left[X_{j}(\hbar \widetilde{r}-\hbar D)_{\rho_{-}^{(j)}}-(\hbar \widetilde{r}-\hbar D)_{\rho_{+}^{(j)}}\right] \mathcal{J}_{R}=0} \\
{\left[X_{j}(\hbar r+\hbar D)_{-\rho_{-}^{(j)}}-(\hbar r+\hbar D)_{-\rho_{+}^{(j)}}\right] \mathcal{K}_{R}=0, \quad j=1, \ldots, n-1 .}
\end{gathered}
$$

Здесь репер $R$ нумерует карту на квантовом листе, $r$ - вершина, а $\left\{\rho^{(j)}\right\}-$ peзонансный базис этого репера, $\widetilde{r}=r-\rho^{(1)}-\cdots-\rho^{(n-1)}$ и $D=\sum_{j=1}^{n-1} \rho^{(j)} X_{j} \frac{\partial}{\partial X_{j}}$. Данная система уравнений для $\mathcal{J}_{R}, \mathcal{K}_{R}$ использовалась в лемме 8.1 для вычисления асимптотик при $\hbar \rightarrow 0$.

В заключение мы покажем, как абстрактное представление резонансной алгебры $\mathcal{A}$ в гильбертовом пространстве $\mathcal{H}$, обладающее вакуумным вектором, и, в частности, представление $(10.11)$, (10.12) сплетаются с универсальными неприводимыми представлениями (13.1).

Зададим в $\mathcal{H}$ когерентные состояния $\mathfrak{P},(12.4)$, и для каждого сечения $\psi$ пучка $\Pi^{*}\left(\Omega_{\hbar}\right)$ определим вектор $\mathcal{P}[\psi] \in \mathcal{H}$ по формуле

$$
\mathcal{P}[\psi] \stackrel{\text { def }}{=} \frac{1}{(2 \pi \hbar)^{n-1}} \int_{\Omega_{\hbar}} \frac{\psi \mathfrak{P}}{\mathcal{K}} d m_{\hbar} .
$$

Отображение

$$
\psi \rightarrow \mathcal{P}[\psi]
$$

назовем когерентным преобразованием.

Теорема 13.2. Когерентное преобразование (13.4) сплетает представление резонансной алгебры $\mathcal{A}$ в гильбертовом пространстве $\mathcal{H}$ с неприводимым представлением в пространстве $\mathcal{L}^{*}\left(\Omega_{\hbar}\right)$ антиголоморфных сечений над квантовым листом $\Omega_{\hbar}=\Omega_{\hbar}[M]$ :

$$
\mathbf{A}_{\sigma} \mathcal{P}[\psi]=\mathcal{P}\left[\stackrel{\circ}{A}_{\sigma} \psi\right], \quad \mathbf{S}_{j} \mathcal{P}[\psi]=\mathcal{P}\left[\stackrel{\circ}{S}_{j} \psi\right]
$$

Здесъ дифберенциалъные операторь $\stackrel{\circ}{A}_{\sigma}, \stackrel{\circ}{S}_{j}$ заданъь формулами (13.1). 
ДокАзАтЕльство. В силу (12.4), (8.1), (8.2), (7.5) когерентное преобразование $\mathcal{P}$ переводит векторы $\overline{U^{t}},(7.1)$, ортонормированного базиса в $\mathcal{L}^{*}\left(\Omega_{\hbar}\right)$ в векторы $\mathfrak{p}^{t} \in \mathcal{H},(13.1)$ :

$$
\mathcal{P}\left[\overline{U^{t}}\right]=\frac{1}{(2 \pi \hbar)^{n-1}} \int_{\Omega_{\hbar}} \frac{\overline{U^{t}}}{\mathcal{K}} \sum_{t^{\prime} \in \Delta[M]} \overline{U^{t^{\prime}}} \mathfrak{p}^{t^{\prime}} d m_{\hbar}=\sum_{t^{\prime} \in \Delta[M]}\left(\overline{U^{t^{\prime}}}, \overline{U^{t}}\right) \mathfrak{p}^{t^{\prime}}=\mathfrak{p}^{t} .
$$

Поэтому достаточно доказать равенства

$$
\mathbf{A}_{\sigma} \mathfrak{p}^{t}=\mathcal{P}\left[\stackrel{\circ}{A_{\sigma}} \overline{U^{t}}\right], \quad \mathbf{S}_{j} \mathfrak{p}^{t}=\mathcal{P}\left[\stackrel{\circ}{S_{j}} \overline{U^{t}}\right] .
$$

Используя (12.3), получим

$$
\mathbf{A}_{\sigma} \mathfrak{p}^{t}=\sqrt{(\hbar t)_{\sigma}} \mathfrak{p}^{t+\sigma}
$$

Сравнивая эту формулу с (13.3) и учитывая, что $P\left[\overline{U^{t}}\right]=\mathfrak{p}^{t}$, получим первое равенство (13.5).

Аналогично, используя формулу (13.2), а также формулу (12.2), получим второе равенство (13.5).

\section{Приложение. Доказательство теоремы 9.2}

Как и при доказательстве теоремы 9.1, мы используем "полярные" координаты $X, \Phi$ и представим квантовую кэлерову форму (8.8) в виде

$$
\omega_{\hbar}=\hbar\left(\frac{\partial g_{1}}{\partial X_{1}} d X_{1} \wedge d \Phi_{1}+\frac{\partial g_{2}}{\partial X_{1}} d X_{1} \wedge d \Phi_{2}+\frac{\partial g_{1}}{\partial X_{2}} d X_{2} \wedge d \Phi_{1}+\frac{\partial g_{2}}{\partial X_{2}} d X_{2} \wedge d \Phi_{2}\right)
$$

где

$$
g_{j} \stackrel{\text { def }}{=} X_{j} \frac{\partial}{\partial X_{j}} \ln \mathcal{K}_{R}
$$

Вычисление интеграла от квантовой формы объема по листу $\Omega$ сводится к вычислению интеграла от первообразной этой 4-формы по границе $\partial \Omega=\bar{\Omega} \cap \partial \mathcal{N}_{0}^{+}$:

$$
\int_{\Omega} \frac{\omega_{\hbar} \wedge \omega_{\hbar}}{2}=\hbar^{2} \int_{\partial \Omega} \theta \wedge d \Phi_{1} \wedge d \Phi_{2}, \quad \theta \stackrel{\text { def }}{=} G_{1} d X_{1}+G_{2} d X_{2} .
$$

Здесь

$$
G_{1}\left(X_{1}, X_{2}\right) \stackrel{\text { def }}{=} g_{2} \frac{\partial g_{1}}{\partial X_{1}}-g_{1} \frac{\partial g_{2}}{\partial X_{1}}, \quad G_{2}\left(X_{1}, X_{2}\right) \stackrel{\text { def }}{=} g_{2} \frac{\partial g_{1}}{\partial X_{2}}-g_{1} \frac{\partial g_{2}}{\partial X_{2}} .
$$

На границе $\partial \Omega$ угловые переменные $\Phi_{j}$ меняются от 0 до $2 \pi$, а радиальные переменные $X_{j}$ меняются вдоль границы классического симплекса (треугольника) $\boldsymbol{\Delta}[\hbar M]$, которая состоит из трех его ребер. Эта граница может попадать на границу координатной карты, поэтому мы разрешим радиальным переменным принимать нулевое и бесконечное значения. В силу (5.13) на ребре, соединяющем первую и вторую вершины, имеем $X_{2}=0$ и, следовательно, $\left.\theta\right|_{\Sigma_{12}}=0$. На ребре, соединяющем вторую и третью вершины, имеем

$$
X_{2}=\infty, \quad X_{1}=\tau X_{2}^{f_{2} / \mu}
$$


и, следовательно,

$$
\left.\theta\right|_{\Sigma_{23}}=\left.\left(X_{2}^{f_{2} / \mu} G_{1}\left(\tau X_{2}^{f_{2} / \mu}, X_{2}\right)\right)\right|_{X_{2}=\infty} d \tau
$$

где $\tau$ - параметр, который при движении от второй вершины к третьей, меняется от 0 до $\infty$. На ребре, соединяющем третью и первую вершины, имеем

$$
X_{1}=\infty, \quad X_{2}=t X_{1}^{-\nu / f_{1}}
$$

и, следовательно,

$$
\left.\theta\right|_{\Sigma_{31}}=\left.\left(X_{1}^{-\nu / f_{1}} G_{2}\left(X_{1}, t X_{1}^{-\nu / f_{1}}\right)\right)\right|_{X_{1}=\infty} d t
$$

где $t$ - параметр, который при движении от третьей вершины к первой меняется от $\infty$ до 0. Таким образом,

$$
\begin{aligned}
\frac{1}{(2 \pi \hbar)^{2}} \int_{\Omega} \frac{\omega_{\hbar} \wedge \omega_{\hbar}}{2}=\frac{1}{2} & \left\{\left.\int_{0}^{\infty}\left(X_{2}^{f_{2} / \mu} G_{1}\left(\tau X_{2}^{f_{2} / \mu}, X_{2}\right)\right)\right|_{X_{2}=\infty} d \tau\right. \\
& \left.-\left.\int_{0}^{\infty}\left(X_{1}^{-\nu / f_{1}} G_{2}\left(X_{1}, t X_{1}^{-\nu / f_{1}}\right)\right)\right|_{X_{1}=\infty} d t\right\} .
\end{aligned}
$$

Вычислим первый интеграл в правой части этого равенства. Используя, как и в доказательстве теоремы 9.1 , явную формулу для полинома $\mathcal{K}_{R}\left(X_{1}, X_{2}\right)$, мы имеем

$$
\mathcal{K}_{R}\left(\tau X_{2}^{f_{2} / \mu}, X_{2}\right)=\sum_{\sigma \in \mathcal{R}_{r}} c_{\sigma}^{r} X_{2}^{f_{2} N_{\sigma}^{(1)} / \mu+N_{\sigma}^{(2)}} \tau^{N_{\sigma}^{(1)}} .
$$

При $X_{2} \rightarrow \infty$ отсюда получаем

$$
\mathcal{K}_{R}\left(\tau X_{2}^{f_{2} / \mu}, X_{2}\right)=\left(\sum_{\sigma \in \mathcal{R}_{r}^{\lambda}} c_{\sigma}^{r} \tau^{N_{\sigma}^{(1)}}\right) X_{2}^{\lambda}+O\left(X_{2}^{\lambda-1}\right) .
$$

Здесь обозначено

$$
\lambda \stackrel{\text { def }}{=} \max _{\sigma \in \mathcal{R}_{r}}\left(\frac{f_{2}}{\mu} N_{\sigma}^{(1)}+N_{\sigma}^{(2)}\right), \quad \mathcal{R}_{r}^{\lambda} \stackrel{\text { def }}{=}\left\{\sigma \in \mathcal{R}_{r} \mid \frac{f_{2}}{\mu} N_{\sigma}^{(1)}+N_{\sigma}^{(2)}=\lambda\right\} .
$$

Дополнительно мы введем краткое обозначение для сумм по множеству $\mathcal{R}_{r}^{\lambda}$ с различными коэффициентами, а именно обозначим

$$
\Sigma\left[\gamma\left(N_{\sigma}^{(1)}\right)\right] \stackrel{\text { def }}{=} \sum_{\sigma \in \mathcal{R}_{r}^{\lambda}} \gamma\left(N_{\sigma}^{(1)}\right) c_{\sigma}^{r} \tau^{N_{\sigma}^{(1)}} .
$$

В частности, формула (П.2) в этих обозначениях имеет вид

$$
\mathcal{K}_{R}\left(\tau X_{2}^{f_{2} / \mu}, X_{2}\right)=\Sigma[1] X_{2}^{\lambda}+O\left(X_{2}^{\lambda-1}\right) .
$$

Аналогично получим

$$
\begin{aligned}
\left(D_{1} \mathcal{K}_{R}\right)\left(\tau X_{2}^{f_{2} / \mu}, X_{2}\right) & =\Sigma\left[N_{\sigma}^{(1)}\right] X_{2}^{\lambda}+O\left(X_{2}^{\lambda-1}\right) \\
\left(D_{2} \mathcal{K}_{R}\right)\left(\tau X_{2}^{f_{2} / \mu}, X_{2}\right) & =\Sigma\left[\lambda-\frac{f_{2}}{\mu} N_{\sigma}^{(1)}\right] X_{2}^{\lambda}+O\left(X_{2}^{\lambda-1}\right), \\
\left(D_{1}^{2} \mathcal{K}_{R}\right)\left(\tau X_{2}^{f_{2} / \mu}, X_{2}\right) & =\Sigma\left[\left(N_{\sigma}^{(1)}\right)^{2}\right] X_{2}^{\lambda}+O\left(X_{2}^{\lambda-1}\right), \\
\left(D_{1} D_{2} \mathcal{K}_{R}\right)\left(\tau X_{2}^{f_{2} / \mu}, X_{2}\right) & =\Sigma\left[N_{\sigma}^{(1)}\left(\lambda-\frac{f_{2}}{\mu} N_{\sigma}^{(1)}\right)\right] X_{2}^{\lambda}+O\left(X_{2}^{\lambda-1}\right),
\end{aligned}
$$


где $D_{j}=X_{j} \frac{\partial}{\partial X_{j}}$. Подставляя эти асимптотики в формулу для $G_{1}$ :

$$
G_{1}\left(X_{1}, X_{2}\right)=\frac{D_{2} \mathcal{K}_{R} \cdot D_{1}^{2} \mathcal{K}_{R}-D_{1} D_{2} \mathcal{K}_{R} \cdot D_{1} \mathcal{K}_{R}}{X_{1}\left(\mathcal{K}_{R}\right)^{2}}
$$

получим

$$
\begin{aligned}
& \left.\left(X_{2}^{f_{2} / \mu} G_{1}\left(\tau X_{2}^{f_{2} / \mu}, X_{2}\right)\right)\right|_{X_{2}=\infty} \\
& =\frac{\Sigma\left[\lambda-\frac{f_{2}}{\mu} N_{\sigma}^{(1)}\right] \Sigma\left[\left(N_{\sigma}^{(1)}\right)^{2}\right]-\Sigma\left[N_{\sigma}^{(1)}\left(\lambda-\frac{f_{2}}{\mu} N_{\sigma}^{(1)}\right)\right] \Sigma\left[N_{\sigma}^{(1)}\right]}{\tau(\Sigma[1])^{2}} \\
& =\lambda \frac{\Sigma[1] \Sigma\left[\left(N_{\sigma}^{(1)}\right)^{2}\right]-\left(\Sigma\left[N_{\sigma}^{(1)}\right]\right)^{2}}{\tau(\Sigma[1])^{2}}=\lambda \frac{\partial}{\partial \tau}\left(\frac{\Sigma\left[N_{\sigma}^{(1)}\right]}{\Sigma[1]}\right) .
\end{aligned}
$$

Поэтому искомый интеграл примет вид

$$
\begin{aligned}
\left.\int_{0}^{\infty}\left(X_{2}^{f_{2} / \mu} G_{1}\left(\tau X_{2}^{f_{2} / \mu}, X_{2}\right)\right)\right|_{X_{2}=\infty} d \tau & =\left.\lambda \frac{\Sigma\left[N_{\sigma}^{(1)}\right]}{\Sigma[1]}\right|_{\tau=0} ^{\infty} \\
& =\lambda\left(\max _{\sigma \in \mathcal{R}_{r}^{\lambda}} N_{\sigma}^{(1)}-\min _{\sigma \in \mathcal{R}_{r}^{\lambda}} N_{\sigma}^{(1)}\right) .
\end{aligned}
$$

Обозначим $l=r^{(2)}+\sigma$. Тогда в силу (7.11) имеем

$$
\sigma \in \mathcal{R}_{r} \Longleftrightarrow l \in \mathbb{Z}_{+}^{3} \text {. }
$$

Учтем явные формулы (5.9) для базиса $\rho^{(1)}, \rho^{(2)}$ и представление (4.3):

$$
\begin{gathered}
l_{1}=r_{1}^{(2)}+N_{\sigma}^{(1)} f_{2}+N_{\sigma}^{(2)} \mu, \\
l_{2}=r_{2}^{(2)}-N_{\sigma}^{(1)} f_{1}+N_{\sigma}^{(2)} \nu, \\
l_{3}=r_{3}^{(2)}+N_{\sigma}^{(2)} .
\end{gathered}
$$

Первое равенство (П.5) и определение (П.3) дают

$$
\lambda=\frac{1}{|\mu|}\left(r_{1}^{(2)}-\min _{l \in \Delta[M]} l_{1}\right)=\frac{r_{1}^{(2)}-r_{1}^{(3)}}{|\mu|},
$$

где мы учли формулы (6.6a): $\min _{l \in \Delta[M]} l_{1}=r_{1}^{(3)}$.

Далее, первое и третье равенства (П.5) дают

$$
N_{\sigma}^{(1)}=\frac{|\mu|}{f_{2}}\left(l_{3}-r_{3}^{(2)}\right)+\frac{1}{f_{2}}\left(l_{1}-r_{1}^{(2)}\right) .
$$

Отсюда получаем

$$
\begin{gathered}
\max _{\sigma \in \mathcal{R}_{r}^{\lambda}} N_{\sigma}^{(1)}=\frac{|\mu|}{f_{2}} \max _{\substack{l \in \Delta[M] \\
l_{1}=r_{1}^{(3)}}} l_{3}+\frac{1}{f_{2}}\left(r_{1}^{(3)}-r_{1}^{(2)}-|\mu| r_{3}^{(2)}\right), \\
\min _{\sigma \in \mathcal{R}_{r}^{\lambda}} N_{\sigma}^{(1)}=\frac{|\mu|}{f_{2}} \min _{\substack{l \in \Delta[M] \\
l_{1}=r_{1}^{(3)}}} l_{3}+\frac{1}{f_{2}}\left(r_{1}^{(3)}-r_{1}^{(2)}-|\mu| r_{3}^{(2)}\right) .
\end{gathered}
$$

Теперь нам понадобится следующая лемма. 
Лемма П.1. Пусть $(j, k, s)$ - какая-то ииклическая перестановка чисел $(1,2,3)$. Обозначим через $l^{*}$ точку, на которой достигается $\min _{l \in \Delta[M]} l_{s}$. Тогда имеют место следующие равенства:

$$
\frac{1}{f_{k}}\left(\max _{\substack{l \in \Delta[M] \\ l_{s}=l_{s}^{*}}} l_{j}-\min _{\substack{l \in \Delta[M] \\ l_{s}=l_{s}^{*}}} l_{j}\right)=\left[\frac{l_{j}^{*}}{f_{k}}\right]+\left[\frac{l_{k}^{*}}{f_{j}}\right]=\left[\frac{r_{k}^{(k)}}{f_{j}}\right],
$$

где $r^{(k)}$ - вериина (6.6а) диофантова остова $\boldsymbol{\Delta}[M]$.

В силу леммы П.1 имеем

$$
\max _{\sigma \in \mathcal{R}_{r}^{\lambda}} N_{\sigma}^{(1)}-\min _{\sigma \in \mathcal{R}_{r}^{\lambda}} N_{\sigma}^{(1)}=\frac{|\mu|}{f_{2}}\left(\max _{\substack{l \in \Delta[M] \\ l_{1}=r_{1}^{(3)}}} l_{3}-\min _{\substack{l \in \Delta[M] \\ l_{1}=r_{1}^{(3)}}} l_{3}\right)=|\mu|\left[\frac{r_{3}^{(3)}}{f_{2}}\right] .
$$

Учитывая последнюю формулу, а также (П.6), мы получаем из (П.4), что

$$
\left.\int_{0}^{\infty}\left(X_{2}^{f_{2} / \mu} G_{1}\left(\tau X_{2}^{f_{2} / \mu}, X_{2}\right)\right)\right|_{X_{2}=\infty} d \tau=\left(r_{1}^{(2)}-r_{1}^{(3)}\right)\left[\frac{r_{3}^{(3)}}{f_{2}}\right] .
$$

Аналогично вычисляется интеграл

$$
-\left.\int_{0}^{\infty}\left(X_{1}^{-\nu / f_{1}} G_{2}\left(X_{1}, t X_{1}^{-\nu / f_{1}}\right)\right)\right|_{X_{1}=\infty} d t=\left(r_{2}^{(2)}-r_{2}^{(1)}\right)\left[\frac{r_{1}^{(1)}}{f_{3}}\right] .
$$

Складывая эти два интеграла в (П.1), приходим к (9.4).

Если в представлении (6.5) все три числа $M_{12}, M_{23}$ и $M_{31}$ неотрицательны, то координаты вершин $r^{(s)}$ в (9.4) задаются формулами $(6.6 \mathrm{~b})$. Подставляя их в (9.4), получим (9.4a).

\section{Список литературы}

1. В. М. Бабич, В.С. Булдырев, Асимптотические методы в задачах дифракции коротких волн, Наука, М., 1972; англ. пер.: V. M. Babic, V.S. Buldyrev, Short-wavelength diffraction theory. Asymptotic methods, Springer Ser. Wave Phenomena, 4, Springer-Verlag, Berlin, 1991.

2. Н. Н. Боголюбов, Н. Н. Боголюбов (мл.), Введение в квантовую статистическую механику, Наука, М., 1984; англ. пер.: N. N. Bogolubov, N. N. Bogolubov, jr., An introduction to quantum statistical mechanics, Gordon and Breach, Yverdon, 1994.

3. Х. Цикон, Р. Фрёзе, В. Кирш, Б. Саймон, Операторы Шрёдингера с приложениями к квантовой механике и глобальной геометрии, Мир, М., 1990; пер. с англ.: H. L. Cycon, R. G. Froese, W. Kirsch, B. Simon, Schrödinger operators with application to quantum mechanics and global geometry, Texts Monogr. Phys., Springer-Verlag, Berlin, 1987.

4. Дж. Биркгоф, Динамические системы, Гостехиздат, М.-Л., 1941; пер. с англ.: G. D. Birkhoff, Dynamical systems, Amer. Math. Soc., New York, 1927.

5. F. G. Gustavson, "Oil constructing formal integrals of a Hamiltonian system near ail equilibrium point", Astron. J., 71 (1966), 670-686.

6. В.И. Арнольд, В. В. Козлов, А. И. Нейштадт, "Математические аспекты классической и небесной механики", Динамические системы - 3, Итоги науки и техн. Сер. Соврем. пробл. мат. Фундам. направления, 3, ВИНИТИ, М., 1985, 5-290; англ. пер.: V.I. Arnold, V.V. Kozlov, A. I. Neishtadt, "Mathematical aspects of 
classical and celestial mechanics", Dynamical systems, III, Encyclopaedia Math. Sci., 3, Springer-Verlag, Berlin, 1988, 1-291.

7. Á.S. Egilsson, "On embedding the $1: 1: 2$ resonance space in a Poisson manifold", Electron. Res. Announc. Amer. Math. Soc., 1:2 (1995), 48-56.

8. B. L. Davis, "Embedding dimensions of Poisson spaces", Int. Math. Res. Not., 2002, № 34, 1805-1839.

9. A. S. Egilsson, Newton polyhedra and Poisson structures from certain linear Hamiltonian circle actions, arXiv: abs/math/0411398.

10. M. Karasev, "Noncommutative algebras, nano-structures, and quantum dynamics generated by resonances. II", Adv. Stud. Contemp. Math. (Kyungshang), 11:1 (2005), $33-56$.

11. М.В. Карасев, "Резонансы и квантовый метод характеристик", Международ. конф. "Дифференциальные уравнения и смежные вопросы" (Москва, 2004), МГУ, M., 2004, 99-100; M. V. Karasev, "Birkhoff resonances and quantum ray method", Proceeding of International Seminar "Day on Diffraction", St.Petersburg University and Steklov Mathematical Institute, St.Petersburg, 2004, 114-126.

12. M. Karasev, "Noncommutative algebras, nanostructures, and quantum dynamics generated by resonances", Quantum algebras and Poisson geometry in mathematical physics, Amer. Math. Soc. Transl. Ser. 2, 216, Amer. Math. Soc., Providence, RI, 2005, 1-17.

13. M. Karasev, "Noncommutative algebras, nano-structures, and quantum dynamics generated by resonances. III", Russ. J. Math. Phys., 13:2 (2006), 131-150.

14. M. Karasev, "Resonance gyrons and quantum geometry", From geometry to quantum mechanics, Progr. Math., 252, Birkhäuser, Boston, 2007, 253-275.

15. А. А. Кириллов, Элементы теории представлений, Наука, М., 1972; англ. пер.: A. A. Kirillov, Elements of the theory of representations, Springer-Verlag, Berlin-New York, 1976.

16. В.П. Маслов, "Применение метода упорядоченных операторов для получения точных решений", ТМФ, 33:2 (1977), 185-209; англ. пер.: V.P. Maslov, "Application of the method of ordered operators to obtain exact solutions", Theoret. and Math. Phys., 33:2 (1977), 960-976.

17. М.В. Карасев, “Асимптотический спектр и фронт осцилляций для операторов с нелинейными коммутационными соотношениями", Докл. АН CCCP, 243:1 (1978), 15-18; англ. пер.: M. V. Karasev, "The asymptotic spectrum and oscillation front for operators with nonlinear commutation relations", Soviet Math. Dokl., 19:6 (1978), 1300-1304.

18. Л. Д. Фаддеев, "Квантовые вполне интегрируемые модели теории поля", Проблемы квантовой теории поля, Дубна, 1979, 249-299.

19. В. П. Маслов, В. Е. Назайкинский, "Алгебры с общими перестановочными соотношениями и их приложения. І. Псевдодифференциальные уравнения с растущими коэффициентами", Итоги науки и техн. Сер. Соврем. пробл. мат., 13, ВИНИТИ, M., 1979, 5-144; англ. пер.: V. P. Maslov, V.E. Nazaikinskii, "Algebras with general commutation relations and their applications. I. Pseudodifferential equations with increasing coefficients", J. Soviet Math., 15:3 (1981), 167-273.

20. М.В.Карасев, В. П. Маслов, “Алгебры с общими перестановочными соотношениями и их приложения. II. Операторные унитарно-нелинейные уравнения", Итоги науки и техн. Сер. Соврем. пробл. мат., 13, ВИНИТИ, М., 1979, 145-267; англ. пер.: M. V. Karasev, V.P. Maslov, "Algebras with general commutation relations and their applications. II. Unitary-nonlinear operator equations", J. Soviet Math., 15:3 (1981), 167-273.

21. Е.К. Склянин, "О некоторых алгебраических структурах, связанных с уравнением Янга-Бакстера. Представления квантовой алгебры”, Функи. анализ и его 
прил., 17:4 (1983), 34-48; англ. пер.: E. K. Sklyanin, "Some algebraic structures connected with the Yang-Baxter equation. Representations of quantum algebras", Funct. Anal. Appl., 17:4 (1983), 273-284.

22. Л. А. Тахтаджян, Л. Д. Фаддеев, Гамильтонов подход в теории солитонов, Наука, M., 1986; англ. пер.: L. D. Faddeev, L. A. Takhtadzhyan, Hamiltonian methods in the theory of solitons, Springer Ser. Soviet Math., Springer-Verlag, Berlin, 1987.

23. Н. Ю. Решетихин, Л.А. Тахтаджян, Л. Д. Фаддеев, "Квантование групп Ли и алгебр Ли", Алгебра и анализ, 1:1 (1989), 178-206; англ. пер.: N. Yu. Reshetikhin, L. A. Takhtadzhyan, L.D. Faddeev, "Quantization of Lie groups and Lie algebras", Leningrad Math. J., 1:1 (1990), 193-225.

24. М. В. Карасев, В.П. Маслов, Нелинейные скобки Пуассона. Геометрия и квантование, Наука, М., 1991; англ. пер.: M. V. Karasev, V.P. Maslov, Nonlinear Poisson brackets. Geometry and quantization, Transl. Math. Monogr., 119, Amer. Math. Soc., Providence, RI, 1993.

25. M. Karasev, E. Novikova, "Non-Lie permutation relations, coherent states, and quantum embedding", Coherent transform, quantization, and Poisson geometry, Amer. Math. Soc. Transl. Ser. 2, 187, Amer. Math. Soc., Providence, RI, 1998, 1-202.

26. М.В. Карасев, Е.М. Новикова, "Квадратичные скобки Пуассона в эффекте Зеемана. Неприводимые представления и когерентные состояния", УМН, 49:5 (1994), 169-170; англ. пер.: M. V. Karasev, E. M. Novikova, "Quadratic Poisson brackets in the Zeeman effect. Irreducible representations and coherent states", Russian Math. Surveys, 49:5 (1994), 179-180.

27. М. В. Карасев, Е. М. Новикова, "Представление точных и квазиклассических собственных функций через когерентные состояния. Атом водорода в магнитном поле", ТМФ, 108:3 (1996), 339-387; англ. пер.: M. V. Karasev, E. M. Novikova, "Representation of exact and semiclassical eigenfunctions via coherent states. Hydrogen atom in a magnetic field", Theoret. and Math. Phys., 108:3 (1996), 1119-1159.

28. М. В. Карасев, Е. М. Новикова, “Алгебра с полиномиальными коммутационными соотношениями для эффекта Зеемана-Штарка в атоме водорода", ТМФ, 142:3 (2005), 530-555; англ. пер.: M. V. Karasev, E. M. Novikova, "Algebra with polynomial commutation relations for the Zeeman-Stark effect in the hydrogen atom", Theoret. and Math. Phys., 142:3 (2005), 447-469; M. Karasev, E. Novikova, "Algebras with polynomial commutation relations for a quantum particle in electric and magnetic fields", Quantum algebras and Poisson geometry in mathematical physics, Amer. Math. Soc. Transl. Ser. 2, 216, Providence, RI, 2005, 19-135.

29. В.В. Козлов, Симметрии, топология и резонансы в гамилътоновой механике, Изд-во УдГУ, Ижевск, 1995; англ. пер.: V.V. Kozlov, Symmetries, topology and resonances in Hamiltonian mechanics, Ergeb. Math. Grenzgeb. (3), 31, Springer-Verlag, Berlin, 1996.

30. G.W. Schwartz, "Smooth functions invariant under the action of a compact Lie group", Topology, 14:1 (1975), 63-68.

31. M. Karasev, "Advances in quantization: quantum tensors, explicit star-products, and restriction to irreducible leaves", Differential Geom. Appl., 9:1-2 (1998), 89-134.

32. M. V. Karasev, "Quantum surfaces, special functions, and the tunneling effect", Lett. Math. Phys., 56:3 (2001), 229-269.

33. В. В. Козлов, Общая теория вихрей, Изд-во УдГУ, Ижевск, 1998; англ. пер.: V. V. Kozlov, Dynamical systems X. General theory of vortices, Encyclopaedia Math. Sci., 67, Springer-Verlag, Berlin, 2003.

34. Б. Костант, "Квантование и унитарные представления", УМН, 28:1 (1973), 163-225; пер. с англ.: В. Kostant, "Quantization and unitary representations. I. Prequantization", Lectures in modern analysis and applications. III, Lecture Notes in Math., 170, Springer, Berlin, 1970, 87-208. 
35. E. M. Novikova, "Minimal basis of the symmetry algebra for three-frequency resonance", Russ. J. Math. Phys., 16:4 (2009), 518-528.

36. В.А. Фок, Работы по квантовой теории поля, Изд-во ЛГУ, Л., 1957.

37. S. Bergman, The Kernel function and conformal mapping, Math. Surveys, 5, Amer. Math. Soc., Providence, RI, 1950.

38. Sh. Chern, "Scientific report on the second summer institute, several complex variables. Part II. Complex manifolds", Bull. Amer. Math. Soc., 62:2 (1956), 101-117.

39. Ф.А. Березин, Метод вторичного квантования, 2-е изд., Наука, М., 1986; англ. пер. 1-го изд.: F. A. Berezin, The method of second quantization, Academic Press, New York-London, 1966.

40. А. М. Переломов, Обобщенные когерентные состояния и их применения, Наука, M., 1987; англ. пер.: A. Perelomov, Generalized coherent states and their applications, Texts Monogr. Phys., Springer-Verlag, Berlin, 1986.

M. B. Kapaceb (M. V. Karasev)

Московский государственный институт

электроники и математики

E-mail: karasev@amath.msk.ru

Е. М. Новикова (Е. M. Novikova)

Московский государственный институт

электроники и математики

E-mail: novikova@miem.edu.ru
Поступило в редакцию 22.04 .2009

28.08 .2009 